\title{
PERIOD IDENTITIES OF CM FORMS ON QUATERNION ALGEBRAS
}

\author{
CHARLOTTE CHAN \\ Department of Mathematics, University of Michigan, 530 Church St., Ann Arbor 48109, USA; \\ email: charchan@umich.edu
}

Received 30 May 2019; accepted 24 February 2020

\begin{abstract}
Waldspurger's formula gives an identity between the norm of a torus period and an $L$-function of the twist of an automorphic representation on GL(2). For any two Hecke characters of a fixed quadratic extension, one can consider the two torus periods coming from integrating one character against the automorphic induction of the other. Because the corresponding $L$-functions agree, (the norms of) these periods - which occur on different quaternion algebras - are closely related. In this paper, we give a direct proof of an explicit identity between the torus periods themselves.
\end{abstract}

2010 Mathematics Subject Classification: 11F67, 11F27, 11R52

\section{Contents}

1 Introduction $\quad 2$

2 Definitions 5

$\begin{array}{lll}3 & \text { Weil representations } & 7\end{array}$

4 Waldspurger, Tunnell-Saito, and a pair of quaternion algebras $\quad 10$

5 Splittings for unitary similitude groups $\quad 14$

6 Global theta lifts $\quad 36$

7 Special vectors in the Weil representation $\quad 57$

(C) The Author 2020. This is an Open Access article, distributed under the terms of the Creative Commons Attribution licence (http://creativecommons.org/licenses/by/4.0/), which permits unrestricted re-use, distribution, and reproduction in any medium, provided the original work is properly cited. 
8 An explicit Rallis inner product formula

9 An example: the canonical Hecke character for $\mathbb{Q}(\sqrt{-7})$

References

\section{Introduction}

Waldspurger's work in 1985 sparked the beginnings of a rich theory studying the relationship between special values of $L$-functions and automorphic periods. In [W85a], he studies torus periods for representations of $B_{\mathbb{A}}^{\times}$, where $B$ is a quaternion algebra over a number field $F$. Consider

$$
\mathscr{P}\left(\pi^{B}, \Omega\right): \pi^{B} \rightarrow \mathbb{C}, \quad f^{B} \mapsto \int_{T_{\mathbb{Q}} \backslash T_{\mathbb{A}}} f^{B}(g) \cdot \Omega(g) d g,
$$

where $\pi^{B}$ is the Jacquet-Langlands transfer of an irreducible automorphic representation $\pi$ of $\mathrm{GL}_{2}\left(\mathbb{A}_{F}\right)$ and $\Omega$ is a character of a maximal torus $T$. Waldspurger establishes a formula

$$
\left|\mathscr{P}\left(f^{B}, \Omega\right)\right|^{2}=* \cdot L\left(\mathrm{BC}(\pi) \otimes \Omega, \frac{1}{2}\right),
$$

where $*$ consists of factors that depend only on local data. Combining Waldspurger's formula with Tunnell-Saito's work on $\epsilon$-dichotomy, which characterizes the branching behavior of representations of local quaternion algebras in terms of local $\epsilon$-factors, one sees that there is at most one quaternion algebra $B$ such that $*$ is nonzero. If $L\left(\mathrm{BC}(\pi) \otimes \Omega, \frac{1}{2}\right) \neq 0$ and the central character condition

$$
\left.\omega_{\pi} \cdot \Omega\right|_{\mathbb{A}_{F}^{\times}}=1, \quad \text { where } \omega_{\pi} \text { is the central character of } \pi,
$$

holds, then there is a unique quaternion algebra $B$-characterized by local $\epsilon$-factors-such that the linear functional $\mathscr{P}\left(\pi^{B}, \Omega\right)$ is nonzero.

In this paper, we will consider the torus periods arising from two symmetric special cases of this: fixing two Hecke characters $\chi_{1}, \chi_{2}$ of $E^{\times}$, consider

(1) $\pi=\pi_{\chi_{1}}$ and $\Omega=\chi_{2}$;

(2) $\pi=\pi_{\chi_{2}}$ and $\Omega=\chi_{1}$.

As such, the only automorphic representations of $\mathrm{GL}_{2}$ we will consider are those that arise as the automorphic induction $\pi_{\chi}$ of a Hecke character $\chi$. As the central 
character of $\pi_{\chi}$ is $\left.\chi\right|_{\mathbb{A}_{F}^{\times}} \cdot \epsilon_{E / F}$, the analogue of the central character condition for both (1) and (2) is

$$
\left.\left.\chi_{1}\right|_{\mathbb{A}_{F}^{\times}} \cdot \chi_{2}\right|_{\mathbb{A}_{F}^{\times}} \cdot \epsilon_{E / F}=1 .
$$

Formally, the Rankin-Selberg $L$-function for the $\left(\mathrm{GL}_{2} \times \mathrm{GL}_{2}\right)$-representation $\pi_{\chi_{1}} \otimes \pi_{\chi_{2}}$ satisfies

$$
L\left(\mathrm{BC}\left(\pi_{\chi_{1}}\right) \otimes \chi_{2}, s\right)=L\left(\pi_{\chi_{1}} \otimes \pi_{\chi_{2}}, s\right)=L\left(\mathrm{BC}\left(\pi_{\chi_{2}}\right) \otimes \chi_{1}, s\right) .
$$

On the other hand, as we see in Equation (1.1), Waldspurger's formula relates (1) to the left-hand side of (1.3) and (2) to the right-hand side of (1.3). Furthermore, the quaternion algebras $B_{1}$ and $B_{2}$ arising from (1) and (2) are related by the following simple formula:

The ramification of $B_{1}$ and $B_{2}$ at a place $v$ agrees if and only if $-1 \in \operatorname{Nm}\left(E_{v}^{\times} / F_{v}^{\times}\right)$.

Therefore one obtains a relationship between (the norms of) the torus periods arising from our two symmetric cases.

As these torus periods occur on different quaternion algebras, it is of interest to study these periods directly, without invoking Waldspurger. In this paper, we do exactly this: we prove an explicit identity between the periods on $B_{1}$ and $B_{2}$. We will employ the theta correspondence to construct automorphic forms and compare the resulting torus periods. To this end, the key to our approach is the construction of a seesaw of dual reductive pairs that precisely realizes the quaternion algebras $B_{1}$ and $B_{2}$.

MAIN THEOREM (6.17). There exist explicitly constructed pairs of automorphic forms $f_{1}^{B_{1}} \in \mathrm{JL}^{B_{1}^{\times}}\left(\pi_{\chi_{1}}\right)$ and $f_{2}^{B_{2}} \in \mathrm{JL}_{2}^{B_{2}^{\times}}\left(\pi_{\chi_{2}}\right)$ such that

$$
\mathscr{P}\left(f_{1}^{B_{1}}, \chi_{2}\right)=\mathscr{P}\left(f_{2}^{B_{2}}, \chi_{1}\right) .
$$

We point out the simplest interesting case of the Main Theorem. Let $F=\mathbb{Q}$ and $E=\mathbb{Q}(\sqrt{-7})$, and consider the canonical Hecke character $\chi_{\text {can }}$ of $E$ in the sense of Rohrlich [Ro80]. Since $\chi_{\text {can }}$ restricts to the quadratic character, $\chi_{1}=\chi_{\text {can }}^{n}$ and $\chi_{2}=\chi_{\text {can }}^{m}$ satisfy (1.2) so long as $n$ and $m$ have opposite parity. When $n=2$ and $m=3+2 l \geqslant 3, B_{1}$ is the split quaternion algebra $M_{2}(\mathbb{Q})$ and $B_{2}$ is the definite quaternion algebra $B$ ramified at exactly 7 and $\infty$. The newform $f$ in the automorphic induction $\pi_{\chi_{\text {can }}^{2}}$ has weight 3 and level $\Gamma_{1}(7)$ with nebentypus $\varepsilon_{\mathbb{Q}(\sqrt{-7}) / \mathbb{Q}}$, and $\delta_{3}^{l} f$ is a test vector for the torus period against $\chi_{\text {can }}^{3+2 l}$, where $\delta_{3}^{l}$ is the $l$ th iterate of the Shimura-Maass differential operator. The Main Theorem gives an explicit automorphic form $f_{l}^{B}$ in the Jacquet-Langlands transfer of $\pi_{\chi_{\text {can }}^{3+2 l}}$ 
to a definite quaternion algebra such that

$$
\int_{\left[E^{\times}\right]}\left(\delta_{3}^{l} f\right)(g) \cdot \chi_{\mathrm{can}}^{3+2 l}(g) d g=\int_{\left[E^{\times}\right]} f_{l}^{B}(g) \cdot \chi_{\mathrm{can}}^{2}(g) d g .
$$

As $l$ changes, the $\delta_{3}^{l} f$ live in the same representation, but on the definite side, the representation space containing $f_{l}^{B}$ also varies. This set-up is now primed for arithmetic application: after dividing by a canonical period and taking $p$-adic limits in $l$, the left-hand side of (1.5) is related to logarithms of generalized Heegner cycles via Bertolini-Darmon-Prasanna [BDP13]. Although we do not consider arithmetic consequences of the Main Theorem here, we plan to explore this in future work.

1.1. Outline. We begin by establishing notation and background in Sections 2 and 3. In Section 4, we give a simple description of the relationship between $B_{1}$ and $B_{2}$. We then construct dual reductive pairs $\left(\mathrm{U}_{B}(V), \mathrm{U}_{B}\left(W^{*}\right)\right)$ and $\left(\mathrm{U}_{E}(\operatorname{Res} V), \mathrm{U}_{E}(W)\right)$ that both capture the behavior of $E^{\times} \subset B_{1}^{\times}, B_{2}^{\times}$and also compatibly map into the same symplectic group. The goal of this paper is then to study the following seesaw of similitude unitary groups with respect to the theta correspondence:

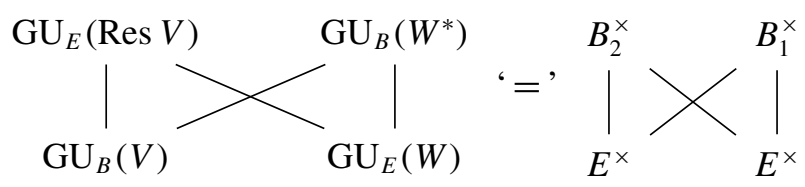

In Section 5, we use Kudla's splittings for unitary groups and explicitly study their compatibility on $E^{\times} \times E^{\times}$. Many of the calculations are similar to the calculations in [IP18++]. From the compatibility statements about the splittings, we can deduce precise information about how the Weil representations on $\mathrm{GU}_{B}(V) \times \mathrm{GU}_{B}\left(W^{*}\right)$ and $\mathrm{GU}_{E}(\operatorname{Res} V) \times \mathrm{GU}_{E}(W)$ are related.

In Section 6, we give a representation theoretic description of the global theta lifts. This requires a careful study of Kudla's splittings at the places $v$ where everything is unramified (Section 5.6). We prove (Theorem 6.1) that the global theta lifts can be described in terms of automorphic induction and JacquetLanglands and that the global theta lift vanishes if and only if the JacquetLanglands transfer does not exist. Combining these results with the compatibility results of Section 5, we obtain our Main Theorem (Theorem 6.17).

In Sections 7 and 8 , in the case $E / F$ is $C M$, we construct a Schwartz function $\varphi$ whose theta lift $\theta_{\varphi}(\chi)$ to $\mathrm{GL}_{2}(F)$ is the newform. We prove an explicit Rallis inner product formula relating $\theta_{\varphi}(\chi)$ to $L(1, \tilde{\chi})$, which in particular shows that 
the theta lift is nonvanishing. These Schwartz functions have been considered in various places before. At the finite places, they have appeared for example in [P06, Proposition 2.5.1], [X07, N1]. At the infinite places, our choice is constructed from a confluent hypergeometric function ${ }_{1} F_{1}(a, b, t)$ of the first type.

We conclude the paper (Section 9) with details on the canonical Hecke character $\chi_{\text {can }}$ of $\mathbb{Q}(\sqrt{-7})$, the example mentioned earlier in Section 1.

\section{Definitions}

For a number field $F$, let $\mathcal{O}$ be the ring of integers of $F$ and $\mathcal{D}$ the different ideal of $F$ over $\mathbb{Q}$. Let $r_{1}$ be the number of real embeddings of $F$ and $2 r_{2}$ be the number of complex embeddings of $F$. For each finite place $v$ of $F$, let $\mathcal{O}_{v}$ be the ring of integers of $F_{v}, \pi_{v}$ a uniformizer of $\mathcal{O}_{v}$, and $q_{v}$ the cardinality of the residue field $\mathcal{O}_{v} / \pi_{v}$. Let $D=D_{F}$ be the discriminant of $F$ and for each finite place $v$ of $F$, let $d_{v}$ be the nonnegative integer such that $\mathcal{D} \otimes_{\mathcal{O}} \mathcal{O}_{v}=\pi_{v}^{d_{v}} \mathcal{O}_{v}$. Set $\delta_{v}=\pi_{v}^{-d_{v}}$. Then $|D|=\prod_{v \nmid \infty} q_{v}^{d_{v}}$.

Throughout this paper, let $E$ be a (possibly split) quadratic extension of $F$ and let $B$ be a quaternion algebra over $F$ containing $E$. The main groups in this paper are $\mathbb{A}_{E}^{\times}, \mathbb{A}_{E}^{1}$, and $B_{\mathbb{A}}^{\times}$. For shorthand, we write

$$
\left[E^{\times}\right]:=\mathbb{A}_{F}^{\times} E^{\times} \backslash \mathbb{A}_{E}^{\times}, \quad\left[E^{1}\right]:=E^{1} \backslash \mathbb{A}_{E}^{1}, \quad\left[B^{\times}\right]:=\mathbb{A}_{F}^{\times} B^{\times} \backslash B_{\mathbb{A}}^{\times},
$$

where in the last definition, we view $\mathbb{A}_{F}^{\times}$as the center of $B_{\mathbb{A}}^{\times}$.

2.1. Measures. Throughout this paper, all integrations over adelic groups are performed with respect to the Tamagawa measure. We define $d x=\prod_{v} d x_{v}$ to be the measure on $\mathbb{A}_{F}$ that is self-dual with respect to a chosen additive character $\psi$ of $F$. We now describe the Tamagawa measure explicitly in a few special cases.

EXAMPLE 2.1. The standard additive character of $F \backslash \mathbb{A}_{F}$ is $\psi:=\psi_{0} \circ \operatorname{Tr}_{F / \mathbb{Q}}$, where $\psi_{0}=\otimes_{v} \psi_{0, v}$ is the nontrivial additive character of $\mathbb{Q} \backslash \mathbb{A}_{\mathbb{Q}}$ given by

$$
\psi_{0, v}(x)= \begin{cases}e^{2 \pi \sqrt{-1} x} & \text { if } v=\infty, \\ e^{-2 \pi \sqrt{-1} x} & \text { if } v \nmid \infty .\end{cases}
$$

Observe that if $v$ is a finite place of $F$, then $\psi_{v}$ is trivial on $\pi_{v}^{-d_{v}} \mathcal{O}_{F_{v}}$ but nontrivial on $\pi_{v}^{-d_{v}-1} \mathcal{O}_{F_{v}}$. The measure $d x$ on $\mathbb{A}_{F}$ that is self-dual with respect to $\psi$ has the property that

- if $v$ is finite, then $\operatorname{vol}\left(\mathcal{O}_{F_{v}}, d x_{v}\right)=q_{v}^{-d_{v} / 2}$;

- if $v$ is infinite, then $d x_{v}$ is the Lebesgue measure. 
More generally, if $\psi^{\prime}$ is any additive character of $\mathbb{A}_{F}$, then for any finite place $v$, we have $\operatorname{vol}\left(\mathcal{O}_{v}, d x_{v}\right)=q_{v}^{c\left(\psi_{v}\right) / 2}$, where $c\left(\psi_{v}\right)$ is the smallest integer such that $\psi_{v}$ is trivial on $\pi_{v}^{c\left(\psi_{v}\right)} \mathcal{O}_{F_{v}}$.

EXAMPLE 2.2. For any number field $k$, put

$$
\rho_{k}:=\operatorname{Res}_{s=1} \zeta_{F}(x)=\frac{2^{r_{1}}(2 \pi)^{r_{2}} h R}{|D|^{1 / 2} w},
$$

where $r_{1}$ is the number of real places of $k, r_{2}$ is the number of complex places of $k, h=h_{k}$ is the class number of $k, R=R_{k}$ is the regulator of $k, D=D_{k}$ is the discriminant of $k$, and $w=w_{k}$ is the number of roots of unity in $k$. Then the Tamagawa measure of $\mathbb{A}_{k}^{\times}$is

$$
d^{\times} x^{\mathrm{Tam}}=\rho_{k}^{-1} \cdot \prod_{v} d^{\times} x_{v}^{\mathrm{Tam}}
$$

where

$$
d^{\times} x_{v}^{\mathrm{Tam}}:= \begin{cases}\left(1-q_{v}^{-1}\right)^{-1} d x_{v} /|x|_{v} & \text { if } v \text { is finite, } \\ d x_{v} /|x|_{v} & \text { if } v \text { is infinite. }\end{cases}
$$

Observe that if $v$ is finite, then $\operatorname{vol}\left(\mathcal{O}_{v}^{\times}, d^{\times} x_{v}^{\text {Tam }}\right)=q_{v}^{-d_{v} / 2}$. The Tamagawa number of $\mathbb{G}_{m}$ is 1 , that is, $\operatorname{vol}\left(k^{\times} \backslash \mathbb{A}_{k}^{\times}, d^{\times} x^{\mathrm{Tam}}\right)=1$.

EXAMPLE 2.3. The previous example explicitly describes the Tamagawa measure of $\mathbb{A}_{F}^{\times}$and $\mathbb{A}_{E}^{\times}$. For each place $v$ of $F$, one has a short exact sequence

$$
1 \rightarrow F_{v}^{\times} \rightarrow E_{v}^{\times} \rightarrow E_{v}^{1} \rightarrow 1,
$$

and hence we may define a local measure $d^{1} g_{v}^{\text {Tam }}$ on $E_{v}^{1}$ as the quotient measure. Then the Tamagawa measure of $E_{\mathbb{A}}^{1}$ is

$$
d^{1} g^{\mathrm{Tam}}:=\frac{\rho_{F}}{\rho_{E}} \cdot \prod_{v} d^{1} x_{v}^{\mathrm{Tam}} .
$$

Observe that if $v$ is a finite place of $F$, then

$$
\operatorname{vol}\left(E_{v}^{1} \cap \mathcal{O}_{E_{v}}^{\times}, d^{1} x_{v}^{\mathrm{Tam}}\right)= \begin{cases}q_{F_{v}}^{-1 / 2} & \text { if } v \text { ramifies in } E, \\ q_{F_{v}}^{-d_{F_{v}} / 2} & \text { if } v \text { is inert or split in } E .\end{cases}
$$

Observe that $\operatorname{vol}\left(E_{v}^{1} \cap \mathcal{O}_{E_{v}}^{\times}, d^{1} x_{v}^{\text {Tam }}\right)=1$ for all but finitely many places $v$. If $F$ is totally real and $E / F$ is totally imaginary, then one can show (for example, by calculating the measure of an annulus in $\mathbb{C}$ containing the unit circle) that

$$
\operatorname{vol}\left(\mathbb{C}^{1}, d^{1} x_{\infty}^{\mathrm{Tam}}\right)=2 \pi
$$


2.2. Conductors. In this section, we briefly review the notion of the conductor of an admissible representation. First, let $k$ be a non-Archimedean local field with a ring of integers $\mathcal{O}_{k}$ and a fixed uniformizer $\pi$. For any integer $N \in \mathbb{Z}_{\geqslant 0}$, let

$$
K_{0}^{\prime}(N):=\left\{\left(\begin{array}{ll}
a & b \\
c & d
\end{array}\right) \in \mathrm{GL}_{2}\left(\mathcal{O}_{k}\right): c \in \pi^{N} \mathcal{O}_{k}\right\}
$$

THEOREM 2.4 (Casselman). Let $\rho$ be an irreducible admissible infinitedimensional representation of $\mathrm{GL}_{2}(k)$ with central character $\omega$. Let $c(\rho) \in \mathbb{Z}_{\geqslant 0}$ be the smallest integer such that

$$
\left\{v \in \rho: \rho(g) v=\omega(a) v \text { for all } g=\left(\begin{array}{ll}
a & b \\
c & d
\end{array}\right) \in K_{0}^{\prime}(c(\rho))\right\} \neq\{0\} .
$$

Then this space has dimension one.

We call $c(\rho)$ the conductor of $\rho$. For a smooth character $\chi: k^{\times} \rightarrow \mathbb{C}^{\times}$, define its conductor $c(\chi) \in \mathbb{Z}_{\geqslant 0}$ to be the smallest number such that $\left.\chi\right|_{U_{k}^{c(x)}}=1$, where $U_{k}^{0}:=\mathcal{O}_{k}^{\times}$and $U_{k}^{n}=1+\pi^{n} \mathcal{O}_{k}$ for $n>0$. Now let $L / k$ be a (possibly split) quadratic extension of $k$. Let $\chi$ be a smooth character of $L^{\times}$and let $\pi_{\chi}$ denote its automorphic induction to $\mathrm{GL}_{2}(k)$. It will be useful for us to have an explicit description of $c\left(\pi_{\chi}\right)$ in terms of $c(\chi)$ for each place $v$ of $F$. This calculation follows from facts about Artin conductors of Galois representations and the fact that conductors of admissible representations of $\mathrm{GL}_{2}(k)$ are compatible with Artin conductors of Galois representations under the local Langlands correspondence. We have

$$
c\left(\pi_{\chi}\right)= \begin{cases}c\left(\chi_{1}\right)+c\left(\chi_{2}\right) & \text { if } L=k \oplus k \text { and } \chi=\chi_{1} \otimes \chi_{2}, \\ c\left(\pi_{\chi}\right)=\operatorname{val}_{k}(4)+2 c(\chi) & \text { if } L / k \text { is unramified, } \\ c\left(\pi_{\chi}\right)=1+\operatorname{val}_{k}(4)+c(\chi) & \text { if } L / k \text { is ramified. }\end{cases}
$$

\section{Weil representations}

Let $k$ be any field. Let $\mathbb{V}$ be a vector space over $k$ with a symplectic form $\langle\langle\cdot, \cdot\rangle\rangle$. The Weil representation of $\operatorname{Sp}(\mathbb{V})$ is a representation of a cover of $\operatorname{Sp}(\mathbb{V})$. It arises in a very natural way, which we briefly recall. The symplectic space $\mathbb{V}$ gives rise to a Heisenberg group $H(\mathbb{V})$, which is a central extension of $\mathbb{V}$ by $k$. The natural action of $\operatorname{Sp}(\mathbb{V})$ on $\mathbb{V}$ extends to an action on $H(\mathbb{V})$ fixing the center $Z(H(\mathbb{V}))=k$. Let $\mathbb{V}=\mathbb{X}+\mathbb{Y}$ be a complete polarization. By the Stone-von Neumann theorem, the irreducible representations of $H(\mathbb{V})$ with nontrivial central character are uniquely determined by their central character and can be realized 
on the vector space $\mathcal{S}(\mathbb{X})$ of Schwartz functions. Thus by Schur's lemma, the $\operatorname{Sp}(\mathbb{V})$ action on $H(\mathbb{V})$ induces an automorphism $\phi_{g}$ of $\mathcal{S}(\mathbb{X})$ that is unique up to scalars. We therefore have a group homomorphism

$$
\left[\omega_{\psi}\right]: \operatorname{Sp}(\mathbb{V}) \rightarrow \operatorname{PGL}(\mathcal{S}(\mathbb{X})), \quad g \mapsto\left[\phi_{g}\right],
$$

where $\left[\phi_{g}\right]$ denotes the image of $\phi_{g}$ under the quotient map $\operatorname{GL}(\mathcal{S}(\mathbb{X})) \rightarrow$ $\operatorname{PGL}(\mathcal{S}(\mathbb{X}))$. This is the projective Weil representation of $\operatorname{Sp}(\mathbb{V})$.

It is natural to try to understand when $\left[\omega_{\psi}\right]$ lifts to a genuine representation of $\operatorname{Sp}(\mathbb{V})$. When $k=\mathbb{F}_{q}$, there exists a lift, but this is not the case in general. The assignment $g \mapsto \phi_{g}$ satisfies

$$
\phi_{g} \phi_{h}=z_{\mathbb{Y}}(g, h) \phi_{g h}, \quad \text { for } g, h \in \operatorname{Sp}(\mathbb{V}) .
$$

It is a straightforward check that $(g, h) \mapsto z_{\mathbb{Y}}(g, h)$ defines a 2-cocycle in $H^{2}\left(\operatorname{Sp}(\mathbb{V}), \mathbb{C}^{\times}\right)$. The 2 -cocycle $z_{\mathbb{Y}}$ corresponds to a central extension $\operatorname{Mp}(\mathbb{V})$ of $\operatorname{Sp}(\mathbb{V})$ and certainly the projective Weil representation of $\operatorname{Sp}(\mathbb{V})$ lifts to a genuine representation of $\operatorname{Mp}(\mathbb{V})$. But we can realize the Weil representation on $\operatorname{Sp}(\mathbb{V})$ itself if and only if $z_{\mathbb{Y}}$ is in fact a 2-coboundary.

In this paper, we will be interested in the adelic Weil representation, which is composed of Weil representations of local fields. For the rest of this section, let $k$ be a local field of characteristic zero, fix an additive character $\psi: k \rightarrow \mathbb{C}^{\times}$, and fix a complete polarization $\mathbb{V}=\mathbb{X}+\mathbb{Y}$.

3.1. Metaplectic groups over local fields. Following [R93, Lemma 3.2], there is an explicit unitary lift $r: \operatorname{Sp}(\mathbb{V}) \rightarrow \operatorname{GL}(\mathcal{S}(\mathbb{X})$ ) (a map of sets) of the projective Weil representation given by

$$
(r(\sigma) \varphi)(x)=\int_{\mathbb{Y} / \operatorname{ker} \gamma} f_{\sigma}(x+y) \varphi(x \alpha+y \gamma) \mu_{\sigma}(d \bar{y})
$$

for any $\varphi \in \mathcal{S}(\mathbb{X})$ and any $\sigma=\left(\begin{array}{ll}\alpha & \beta \\ \gamma & \delta\end{array}\right)$, where $\mu_{\sigma}$ is a Haar measure on $\mathbb{Y} / \operatorname{ker} \gamma, \bar{y}$ is the coset $y+\operatorname{ker} \gamma \in \mathbb{Y} / \operatorname{ker} \gamma$, and $f_{\sigma}(x+y)=\psi\left(q_{\sigma}(x+y)\right)$ for

$$
q_{\sigma}(x+y)=\frac{1}{2}\left\langle\langle x \alpha, x \beta\rangle+\frac{1}{2}\langle\langle y \gamma, y \delta\rangle\rangle+\langle\langle y \gamma, x \beta\rangle .\right.
$$

Moreover, this lift is the unique lift satisfying the properties in [R93, Theorem 3.5]. We then define the 2-cocycle $z_{\mathbb{Y}}: \operatorname{Sp}(\mathbb{V}) \times \operatorname{Sp}(\mathbb{V}) \rightarrow \mathbb{C}^{1}$ by

$$
r(g h)=z_{\mathbb{Y}}(g, h)^{-1} \cdot r(g) \cdot r(h) .
$$

This represents a class in $H^{2}\left(\operatorname{Sp}(\mathbb{V}), \mathbb{C}^{1}\right)$ and therefore gives rise to a $\mathbb{C}^{1}$-extension $\operatorname{Mp}(\mathbb{V})$ of $\operatorname{Sp}(\mathbb{V})$, which we call the metaplectic group. Explicitly, 
this group is the set $\operatorname{Sp}(\mathbb{V}) \times \mathbb{C}^{1}$ together with the multiplication rule

$$
(g, x) \cdot(h, y)=\left(g h, x y \cdot z_{\mathbb{Y}}(g, h)\right) .
$$

We define the Weil representation $\omega_{\psi}$ on the metaplectic group $\operatorname{Mp}(\mathbb{V})$ to be

$$
\omega_{\psi}: \operatorname{Mp}(\mathbb{V}) \rightarrow \operatorname{GL}(\mathcal{S}(\mathbb{X})), \quad(g, z) \mapsto z \cdot r(g) .
$$

Oftentimes, it is easier to work with the following description of $\omega_{\psi}$ :

$$
\begin{aligned}
\omega_{\psi}\left(\left(\begin{array}{ll}
a & \\
& \left(a^{\mathrm{t}}\right)^{-1}
\end{array}\right), z\right) \varphi(x) & =z \cdot|\operatorname{det} a|^{1 / 2} \cdot \varphi(x a) \\
\omega_{\psi}\left(\left(\begin{array}{ll}
\mathbf{1}_{n} & b \\
& \mathbf{1}_{n}
\end{array}\right), z\right) \varphi(x) & =z \cdot \psi\left(\frac{1}{2} x b^{\mathrm{t}} x\right) \cdot \varphi(x) \\
\omega_{\psi}\left(\left(\begin{array}{rr}
\mathbf{1}_{n} & \mathbf{1}_{n} \\
-\mathbf{1}_{n} &
\end{array}\right), z\right) \varphi(x) & =z \cdot \int_{k^{n}} \varphi(y) \psi\left(x^{\mathrm{t}} y\right) d y
\end{aligned}
$$

for $\varphi \in \mathcal{S}(\mathbb{X}), x \in \mathbb{X} \cong k^{n}, a \in \mathrm{GL}(\mathbb{X}) \cong \mathrm{GL}_{n}(k), b \in \operatorname{Hom}(\mathbb{X}, \mathbb{Y}) \cong \mathrm{M}_{n}(k)$ with $b^{\mathrm{t}}=b$, and $z \in \mathbb{C}^{1}$. In (3.3), we take $d y$ to be the product of the self-dual Haar measure on $k$ with respect to $\psi$.

It will later (for example, in Section 7) be convenient to understand how changing the additive character $\psi$ affects the Weil representation $\omega_{\psi}$. One can check that the Weil representation with respect to the additive character $\psi_{v}(x):=$ $\psi(v x)$ satisfies

$$
\omega_{\psi}\left(d(v)^{-1} g d(v), z\right)=\omega_{\psi_{v}}(g, z), \quad \text { where } d(v):=\left(\begin{array}{ll}
1 & 0 \\
0 & v
\end{array}\right) \text { for } v \in k .
$$

If for a subgroup $\iota: G \hookrightarrow \operatorname{Sp}(\mathbb{V})$, the restriction of $z_{\mathbb{Y}}$ represents the trivial class in $H^{2}\left(G, \mathbb{C}^{1}\right)$, then via an explicit trivialization $s$ of $\left.z_{\mathbb{Y}}\right|_{G \times G}$, we can define the Weil representation $\omega_{\psi}$ on $G$ as

$$
\omega_{\psi}: G \rightarrow \operatorname{GL}(\mathcal{S}(\mathbb{X})), \quad g \mapsto \omega_{\psi}(g, s(g)) .
$$

One feature that makes the Weil representation computable is the fact that the 2-cocycle $z_{\mathbb{Y}}$ can be expressed in terms of the Weil index of the Leray invariant. Let $k$ be a local field. For any nontrivial additive character $\psi$ of $k$ and any nondegenerate symmetric $k$-bilinear form $q: \mathbb{V} \times \mathbb{V} \rightarrow k$, we write $\gamma_{F}(\psi \circ q) \in \mu_{8}$ to denote the Weil index associated with the character of second degree $x \mapsto \psi(q(x, x))$ (see [R93, Appendix], [IP18+, Section 3.1.1]). For any maximal isotropic subspaces $\mathbb{Y}, \mathbb{Y}^{\prime}, \mathbb{Y}^{\prime \prime}$ of $\mathbb{V}$, the Leray invariant $q\left(\mathbb{Y}, \mathbb{Y}^{\prime}, \mathbb{Y}^{\prime \prime}\right)$ is a nondegenerate symmetric $k$-bilinear form on $\mathbb{V}$ (see [R93, Sections 2.3,2.4], [IP18+, Section 3.1.2]). Then for any $g_{1}, g_{2} \in \operatorname{Sp}(\mathbb{V})$,

$$
z_{\mathbb{Y}}\left(g_{1}, g_{2}\right)=\gamma_{F}\left(\frac{1}{2} \psi \circ q\left(\mathbb{Y}, \mathbb{Y} g_{2}^{-1}, \mathbb{Y} g_{1}\right)\right) \text {. }
$$


3.2. The doubled Weil representation. Now consider the doubled symplectic space $\mathbb{V}^{\square}:=\mathbb{V}+\mathbb{V}^{-}$, where $\mathbb{V}^{-}$has the negated form. Let $\mathbb{X}^{\square}=\mathbb{X}+\mathbb{X}^{-}, \mathbb{Y}^{\square}=$ $\mathbb{Y}+\mathbb{Y}^{-}$, and let $\omega_{\psi}^{\square}$ denote the Weil representation on the metaplectic group $\operatorname{Mp}\left(\mathbb{V}^{\square}\right)$ with respect to $\mathbb{V}^{\square}=\mathbb{X}^{\square}+\mathbb{Y}^{\square}$. We will also make use of the polarization $\mathbb{V}^{\square}=\mathbb{V}^{\Delta}+\mathbb{V} \nabla$, where $\mathbb{V}^{\Delta}=\{(v, v): v \in \mathbb{V}\}$ and $\mathbb{V} \nabla=\{(v,-v): v \in \mathbb{V}\}$. Identifying $\operatorname{Sp}\left(\mathbb{V}^{-}\right)$with $\operatorname{Sp}(\mathbb{V})^{\text {op }}$, we have a natural homomorphism

$$
\tilde{\iota}: \operatorname{Mp}(\mathbb{V}) \times \operatorname{Mp}(\mathbb{V})^{\mathrm{op}} \rightarrow \operatorname{Mp}\left(\mathbb{V}^{\square}\right), \quad((g, z),(h, w)) \mapsto\left(\operatorname{diag}\left(g, h^{-1}\right), z w^{-1}\right) .
$$

3.3. Dual reductive pairs and the Howe correspondence. A dual reductive pair $\left(G, G^{\prime}\right)$ in $\operatorname{Sp}(\mathbb{V})$ is a pair of reductive subgroups of $\operatorname{Sp}(\mathbb{V})$, which are mutual centralizers of each other. There is a natural map

$$
i: G \times G^{\prime} \rightarrow \operatorname{Sp}(\mathbb{V}), \quad\left(g, g^{\prime}\right) \mapsto\left(v \mapsto g^{-1} v g^{\prime}\right) .
$$

If the cocycle $z_{\mathbb{Y}}$ can be trivialized on $i\left(G \times G^{\prime}\right) \subset \operatorname{Sp}(\mathbb{V})$, we can define the Weil representation on $i\left(G \times G^{\prime}\right)$ and pull back to a Weil representation of $G \times G^{\prime}$. In [K94], Kudla wrote down explicit splittings of $z_{\mathbb{Y}}$. We will make use of this work heavily in the present paper.

The Weil representation $\omega_{\psi}$ on $G \times G^{\prime}$ has the following multiplicity-one property. For an irreducible $G$-representation $\pi$, let $\mathcal{S}(\pi)$ denote the largest quotient of $\mathcal{S}(\mathbb{X})$ such that $G$ acts by $\pi$. By [MVW, Ch. 2, Lemma III.4], there exists a unique irreducible $G^{\prime}$-representation $\Theta(\pi)$ such that

$$
\mathcal{S}(\pi) \cong \pi \otimes \Theta(\pi) .
$$

We call $\Theta(\pi)$ the local theta lift of $\pi$.

\section{Waldspurger, Tunnell-Saito, and a pair of quaternion algebras}

For any quaternion algebra $B$ over $F$, we write $\Sigma_{B}:=$ \{places $v$ of $F$ such that $B_{v}$ is ramified\}.

4.1. Waldspurger's formula. Let $\pi$ be an irreducible cuspidal automorphic representation of $\mathrm{GL}_{2}\left(\mathbb{A}_{F}\right)$ with central character $\omega_{\pi}$ that has a nonzero JacquetLanglands transfer $\pi^{B}$ to $B_{\mathbb{A}}^{\times}$. Recall that this means that $\pi_{v}$ is a discrete series at all $v \in \Sigma_{B}$. Let $\Omega$ be any Hecke character of $E^{\times}$such that $\left.\Omega\right|_{\mathbb{A}_{F}^{\times}}=\omega_{\pi}^{-1}$. Define

$$
\mathscr{P}\left(\pi^{B}, \Omega\right): \pi^{B} \rightarrow \mathbb{C}, \quad f \mapsto \int_{\left[E^{\times}\right]} f(t) \Omega(t) d t .
$$


We have the following classical theorem, which follows from combining Waldspurger's formula with the local $\epsilon$-dichotomy theorem of Tunnell and Saito.

THEOREM 4.1 (Waldspurger [W85a], Tunnell [T83], Saito [S93]). Let $\pi$ be an irreducible cuspidal automorphic representation of $\mathrm{GL}_{2}\left(\mathbb{A}_{F}\right)$ with central character $\omega_{\pi}$. If

$$
L\left(\mathrm{BC}(\pi) \otimes \Omega, \frac{1}{2}\right) \neq 0, \quad \text { and }\left.\quad \Omega\right|_{\mathbb{A}_{F}^{\times}}=\omega_{\pi}^{-1},
$$

then there exists a unique quaternion algebra $B=B_{\pi, \Omega}$ over $F$ such that

$$
\mathscr{P}\left(\pi^{B}, \Omega\right) \neq 0 \text {. }
$$

Moreover, $B$ is the unique quaternion algebra with ramification set

$$
\Sigma_{\pi, \Omega}:=\left\{v: \epsilon_{v}(\mathrm{BC}(\pi) \otimes \Omega) \cdot \omega_{v}(-1)=-1\right\} .
$$

Proof. If $L\left(\mathrm{BC}(\pi) \otimes \Omega, \frac{1}{2}\right) \neq 0$, then $\epsilon(\mathrm{BC}(\pi) \otimes \Omega)=+1$. Since $\omega$ is a Hecke character of $\mathbb{A}^{\times}$, we must have $\omega(-1)=+1$. Therefore, there must be an even number of places $v$ of $F$ such that $\epsilon_{v}(\operatorname{BC}(\pi) \otimes \Omega) \cdot \omega_{v}(-1)=-1$, and hence there exists a unique quaternion algebra $B_{\pi, \Omega}$ over $F$ with ramification set $\Sigma_{\pi, \Omega}$, and the conclusion now follows from Waldspurger's formula and the local branching criterion of Tunnell and Saito.

4.2. A pair of quaternion algebras. We now specialize to the setting where $\pi$ comes from automorphic induction. Let $\chi, \chi^{\prime}$ be Hecke characters of $\mathbb{A}_{E}^{\times}$. One has

$$
L\left(\mathrm{BC}\left(\pi_{\chi}\right) \otimes \chi^{\prime}, s\right)=L\left(\pi_{\chi} \otimes \pi_{\chi^{\prime}}, s\right)=L\left(\mathrm{BC}\left(\pi_{\chi^{\prime}}\right) \otimes \chi, s\right),
$$

and let us assume that

$$
L\left(\mathrm{BC}\left(\pi_{\chi}\right) \otimes \chi^{\prime}, \frac{1}{2}\right)=L\left(\mathrm{BC}\left(\pi_{\chi^{\prime}}\right) \otimes \chi, \frac{1}{2}\right) \neq 0 .
$$

It is a standard calculation to see that the central character of $\pi_{\chi}$ (and of any Jacquet-Langlands transfer $\pi_{\chi}^{B}$ ) is $\left.\chi\right|_{\mathbb{A}_{F}^{\times}} \cdot \epsilon_{E / F}$, where $\epsilon_{E / F}$ is the quadratic character of $\mathbb{A}_{F}^{\times}$associated with the quadratic extension $E / F$. Therefore the central character condition in Theorem 4.1 is

$$
\left.\left.\chi\right|_{\mathbb{A}_{F}^{\times}} \cdot \chi^{\prime}\right|_{\mathbb{A}_{F}^{\times}} \cdot \epsilon_{E / F}=1 .
$$

If $\chi, \chi^{\prime}$ satisfy (4.2), then by Theorem $4.1, B=B_{\pi_{\chi}, \chi^{\prime}}$ and $B^{\prime}=B_{\pi_{\chi^{\prime}}, \chi}$ are the unique quaternion algebras such that $\mathscr{P}\left(\pi_{\chi}^{B}, \chi^{\prime}\right) \neq 0$ and $\mathscr{P}\left(\pi_{\chi^{\prime}}^{B^{\prime}}, \chi\right) \neq 0$. 
Proposition 4.2. Let $\chi, \chi^{\prime}$ be Hecke characters of $\mathbb{A}_{E}^{\times}$satisfying (4.1) and (4.2), and let $E=F(\mathbf{i})$ with $\mathbf{i}^{2}=u \in F^{\times}$. If $B=B_{\pi_{\chi}, \chi^{\prime}}$ is the quaternion algebra that corresponds to the Hilbert symbol $(u, J)$, then $B^{\prime}=B_{\pi_{\chi^{\prime}}, \chi}$ corresponds to the Hilbert symbol $(u,-J)$.

Proof. It is a standard computation to show that

$$
\epsilon_{v}\left(\mathrm{BC}\left(\pi_{\chi}\right) \otimes \chi^{\prime}\right)=\epsilon_{v}\left(\mathrm{BC}\left(\pi_{\chi^{\prime}}\right) \otimes \chi\right) .
$$

Equation (4.2) implies that $\omega_{\pi_{\chi}} \cdot \omega_{\pi_{\chi^{\prime}}} \cdot \epsilon_{E / F}=1$. Using Theorem 4.1, we see that $\Sigma_{\pi_{\chi^{\prime}, \chi}}$ can be described in terms of $\Sigma_{\pi_{\chi}, \chi^{\prime}}$ :

$$
\Sigma_{\pi_{\chi^{\prime}}, \chi}=\left\{v: \begin{array}{l}
v \in \Sigma_{\pi_{\chi}, \chi^{\prime}} \text { and } \epsilon_{E_{v} / F_{v}}(-1)=1 \text {, or } \\
v \notin \Sigma_{\pi_{\chi}, \chi^{\prime}} \text { and } \epsilon_{E_{v} / F_{v}}(-1)=-1 .
\end{array}\right\}
$$

An equivalent way to state this relationship is the following. The quaternion algebra $B$ can be given an $F$ basis $1, \mathbf{i}, \mathbf{j}, \mathbf{i j}$ such that $E=F[\mathbf{i}]$. Write $\mathbf{i}^{2}=u$ and $\mathbf{j}^{2}=J$ so that $B$ is the quaternion algebra associated with the Hilbert symbol $(u, J)$. That is,

$$
(u, J)_{v}=-1 \Longleftrightarrow v \in \Sigma_{\pi_{\chi}, \chi^{\prime}} .
$$

By the bimultiplicativity of the Hilbert symbol, $B^{\prime}$ is the quaternion algebra associated with

$$
(u, J) \cdot \epsilon_{E / F}(-1)=(u, J) \cdot(u,-1)=(u,-J) .
$$

4.3. A seesaw of unitary groups. In this section, we introduce the main dual reductive pairs of interest in this paper. Fix $\mathbf{i} \in E$ with $\operatorname{tr}_{E / F} \mathbf{i}=\mathbf{i}+\overline{\mathbf{i}}=0$. Note that $E=F[\mathbf{i}]$. Let $B$ be a (possibly split) quaternion algebra over $F$ and let $1, \mathbf{i}, \mathbf{j}, \mathbf{k}$ be a standard basis for $B$ over $F$. Viewing $B=E \oplus E \mathbf{j}$, we set pr: $B \rightarrow E$ to be the projection onto the $E$-component. We consider the following spaces:

- $V=B=1$-dimensional right $B$-space with skew-Hermitian form $\langle x, y\rangle=$ $x^{*} \mathbf{i} y$

- $W^{*}=E \otimes_{E} B=1$-dimensional left $B$-space with Hermitian form $(x, y)=x y^{*}$;

- Res $V=2$-dimensional right $E$-space with skew-Hermitian form $\langle x, y\rangle=$ $\operatorname{pr}\left(x^{*} \mathbf{i} y\right)$;

- $W=E=1$-dimensional left $E$-space with Hermitian form $(a, b)=a \bar{b}$;

- $V_{0}=1$-dimensional right $E$-space with Hermitian form $\langle a, b\rangle_{0}=\bar{a} b$; 
- $W_{0}=B=2$-dimensional left $E$-space with skew-Hermitian form $(x, y)_{0}=$ $-\mathbf{i} \operatorname{pr}\left(x y^{*}\right)$;

- $\mathbb{V}=\operatorname{Res}_{B / F}\left(V \otimes_{B} W^{*}\right)=\operatorname{Res}_{E / F}\left(\operatorname{Res} V \otimes_{E} W\right)=\operatorname{Res}_{E / F}\left(V_{0} \otimes_{E} W_{0}\right)=$ 4-dimensional $F$-space with symplectic form $\frac{1}{2} \operatorname{Tr}_{E / F}(\langle\cdot, \cdot\rangle \otimes \overline{(\cdot, \cdot)})$.

Then both pairs $\left(U_{B}(V), U_{B}\left(W^{*}\right)\right)$ and $\left(U_{E}(\operatorname{Res} V), U_{E}(W)\right)$ are irreducible dual reductive pairs (of type 1) in $\operatorname{Sp}(\mathbb{V})$. (See, for example, [P93].) For any pair $(V, W)=\left(V, W^{*}\right),(\operatorname{Res} V, W)$, or $\left(V_{0}, W_{0}\right)$, we take as our convention

$$
\mathrm{GL}(V) \times \mathrm{GL}(W) \rightarrow \mathrm{GL}(V \otimes W), \quad(g, h) \mapsto\left(v \otimes w \mapsto g^{-1} v \otimes w h\right) .
$$

It is clear that $U_{B}(V) \subset U_{E}(\operatorname{Res} V)$ and that $U_{E}(W) \subset U_{B}\left(W^{*}\right)$. Furthermore, we have a commutative diagram

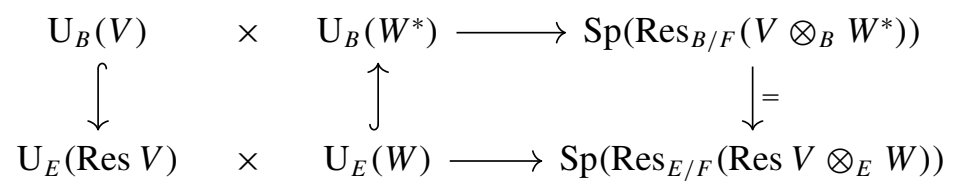

Therefore we have the following seesaw of dual reductive pairs:

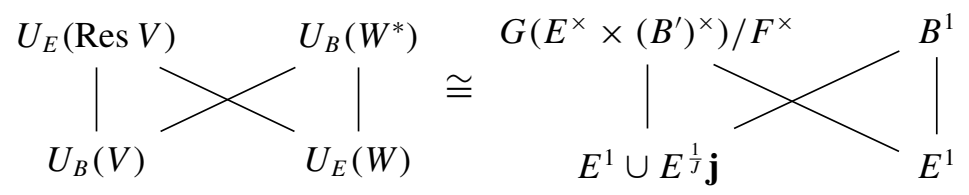

Here, $B^{\prime}=\left(\frac{\mathbf{i}^{2},-\mathbf{j}^{2}}{F}\right)$, the superscript $r \in \mathbb{Q}$ picks out the norm- $r$ elements, and $G\left(E^{\times} \times\left(B^{\prime}\right)^{\times}\right)$is the subgroup of $E^{\times} \times\left(B^{\prime}\right)^{\times}$consisting of elements $(\alpha, \beta)$ with $\operatorname{Nm}_{E / F}(\alpha)=\operatorname{Nrd}_{B^{\prime} / F}(\beta)$. Note that $F^{\times}$maps antidiagonally into $G\left(E^{\times} \times\left(B^{\prime}\right)^{\times}\right)$. The analogous seesaw with similitudes is

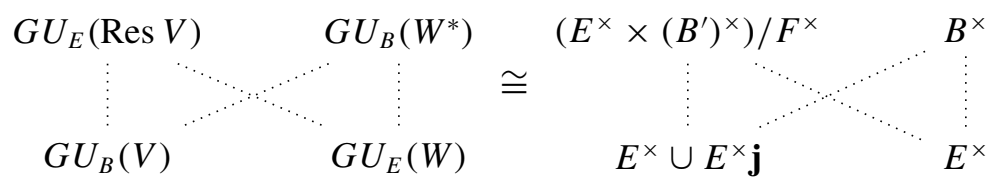

The only isomorphism that is not straightforward to see is $\mathrm{GU}_{E}(\operatorname{Res} V) \cong$ $\left(\left(B^{\prime}\right)^{\times} \times E^{\times}\right) / F^{\times}$. The $E^{\times}$factor comes from the fact that $\operatorname{Res} V$ is a right $E$-space, and the $\left(B^{\prime}\right)^{\times}$factor comes from a natural left action of $\left(B^{\prime}\right)^{\times}$on Res $V=B$ defined by

$$
\mathbf{j}^{\prime} \cdot 1=\mathbf{j}, \quad \mathbf{j}^{\prime} \cdot \mathbf{j}=-J
$$


and extending this action left $E$-linearly. Observe that this action commutes with right multiplication by $E^{\times}$on $\operatorname{Res} V$.

We note that the point of introducing the $E$-spaces $V_{0}$ and $W_{0}$ is that we have natural maps

$$
U_{B}(V)^{0} \cong U_{E}\left(V_{0}\right), \quad U_{B}\left(W^{*}\right) \hookrightarrow U_{E}\left(W_{0}\right)
$$

This will allow us to compute splittings on the quaternionic unitary groups $U_{B}(V)$ and $U_{B}\left(W^{*}\right)$ by pulling back splittings on $U_{E}\left(V_{0}\right)$ and $U_{E}\left(W_{0}\right)$.

\section{Splittings for unitary similitude groups}

Fix an additive character $\psi$ of $\mathbb{A}_{F}$ trivial on $F$. In this section, we define the Weil representation $\omega_{\psi}$ on the dual reductive pairs introduced in Section 4.3 using the explicit splittings of $z_{\mathbb{Y}}$ defined by Kudla [K94]. The properties of the Weil index and the Leray invariant we will use in this section can be found in [R93] and [IP18+, Sections 3.1.1, 3.1.2]. We prove that the splittings are compatible with the seesaws constructed in Section 4.3. In Section 5.5, we combine the local considerations from Sections 5.1-5.4 into the global picture. Many of these calculations (especially in Sections 5.3 and 5.4) are similar to those in [IP18+, Appendix C] and [IP18++].

In order to describe the global automorphic theta lift from a Hecke character to a quaternion algebra, which we will do later in Section 6, we will need to give an explicit description of the local splittings in Section 5.3 in the special case that the quaternion algebra is unramified (that is, split) at the place in question. We do this in Section 5.6.

Notation. In Sections 5.1-5.4 and 5.6, we fix a place $v$ of $F$ and suppress $v$ from the notation so that $E$ is a (possibly split) quadratic extension of a local field $F$. The only subsection in this section where $E / F$ is a quadratic extension of global fields is Section 5.5.

5.1. Kudla's splitting for split unitary groups. We first recall Kudla's splitting [K94] of Rao's cocycle [R93] for split unitary groups over $E$. Let $\mathbf{W} \cong E^{2 n}$ (row vectors) be an $E$-vector space of dimension $2 n$ with $\epsilon$-skew Hermitian form

$$
\left\langle\left(x_{1}, y_{1}\right),\left(x_{2}, y_{2}\right)\right\rangle=x_{1} \bar{y}_{2}^{\mathrm{t}}-\epsilon y_{1} \bar{x}_{2}^{\mathrm{t}},
$$

and let $e_{1}, \ldots, e_{n}, e_{1}^{\prime}, \ldots, e_{n}^{\prime}$ be the $E$-basis of $\mathbf{W}$ giving the isomorphism $\mathbf{W} \cong E^{2 n}$. Let $\mathbf{V}$ be an $E$-vector space of dimension $m$ with a nondegenerate 
$\epsilon$-Hermitian form $(\cdot, \cdot)$. (Here, $\bar{x}$ denotes the image of $x$ under the nontrivial involution of $E$ over $F$ and the superscript ${ }^{t}$ denotes transposition.) Then $\left(\mathrm{U}_{E}(\mathbf{V})\right.$, $\mathrm{U}_{E}(\mathbf{W})$ ) is a dual reductive pair and there is a natural map

$$
\iota: \mathrm{U}_{E}(\mathbf{V}) \times \mathrm{U}_{E}(\mathbf{W}) \rightarrow \mathrm{Sp}\left(\mathbf{V} \otimes_{E} \mathbf{W}\right), \quad(h, g) \mapsto\left(w \otimes v \mapsto h^{-1} w \otimes v g\right) .
$$

We denote by $\iota_{\mathbf{W}}: \mathrm{U}_{E}(\mathbf{V}) \rightarrow \operatorname{Sp}\left(\mathbf{V} \otimes_{E} \mathbf{W}\right)$ and $\iota_{\mathbf{V}}: \mathrm{U}_{E}(\mathbf{W}) \rightarrow \operatorname{Sp}\left(\mathbf{V} \otimes_{E} \mathbf{W}\right)$ the restrictions of $\iota$ to $\mathrm{U}_{E}(\mathbf{V}) \times\{1\}$ and $\{1\} \times \mathrm{U}_{E}(\mathbf{W})$, respectively.

For $0 \leqslant j \leqslant n$, let $\tau_{j} \in \mathrm{U}_{E}(\mathbf{W})$ be the element defined by

$$
e_{i} \tau_{j}=\left\{\begin{array}{ll}
-\epsilon e_{i}^{\prime} & \text { if } 1 \leqslant i \leqslant j, \\
e_{i} & \text { if } i>j,
\end{array} \quad \text { and } \quad e_{i}^{\prime} \tau_{j}= \begin{cases}e_{i} & \text { if } 1 \leqslant i \leqslant j \\
e_{i}^{\prime} & \text { if } i>j\end{cases}\right.
$$

Then

$$
\mathrm{U}_{E}(\mathbf{W})=\bigsqcup_{j=0}^{n} P \tau_{j} P
$$

where $P=P_{Y} \subset \mathrm{U}_{E}(\mathbf{W})$ is the parabolic subgroup stabilizing the maximal isotropic subspace $Y:=\operatorname{span}_{E}\left\{e_{1}^{\prime}, \ldots, e_{n}^{\prime}\right\}$. If $g=p_{1} \tau_{j} p_{2} \in P \tau_{j} P$, then we define

$$
j(g):=j, \quad \text { and } \quad x(g):=\operatorname{det}\left(\left.p_{1} p_{2}\right|_{Y}\right) \in E^{\times} .
$$

For any $m$-dimensional $E$-vector space $\mathbf{V}_{0}$ endowed with a nondegenerate Hermitian form, define

$$
\gamma_{F}\left(\frac{1}{2} \psi \circ R \mathbf{V}_{0}\right):=\left(u, \operatorname{det}\left(\mathbf{V}_{0}\right)\right)_{F} \gamma_{F}\left(-u, \frac{1}{2} \psi\right)^{m} \gamma_{F}\left(-1, \frac{1}{2} \psi\right)^{-m},
$$

where for any $a \in F^{\times}$, we set $\gamma_{F}\left(a, \frac{1}{2} \psi\right):=\gamma_{F}\left(\frac{a}{2} \psi \circ q\right) / \gamma_{F}\left(\frac{1}{2} \psi \circ q\right) \in \mu_{8}$, a quotient of Weil indices.

DEFINITION 5.1. Define

$$
\beta_{\mathbf{V}, \xi}: \mathbf{U}_{E}(\mathbf{W}) \rightarrow \mathbb{C}^{1}, \quad g \mapsto \begin{cases}\xi(x(g)) \gamma_{F}\left(\frac{1}{2} \psi \circ R \mathbf{V}\right)^{-j(g)} & \text { if } \epsilon=+1, \\ \xi(x(g)) \xi(\mathbf{i})^{j} \gamma_{F}\left(\frac{1}{2} \psi \circ R \mathbf{V}^{\prime}\right)^{-j(g)} & \text { if } \epsilon=-1,\end{cases}
$$

where $\mathbf{V}^{\prime}$ is the Hermitian form obtained by scaling the skew-Hermitian form on V by $\mathbf{i}$.

THEOREM 5.2 (Kudla, [K94, Theorem 3.1]). Let $\xi$ be a unitary character of $E^{\times}$whose restriction to $F^{\times}$is $\epsilon_{E / F}^{m}$, where $\epsilon_{E / F}(x)=(x, u)_{F}$ is the quadratic character corresponding to the extension $E / F$. Then for the maximal isotropic subspace $\mathbb{Y}:=\mathbf{V} \otimes_{E} Y$ of $\mathbf{V} \otimes_{E} \mathbf{W}$,

$$
z_{\mathbb{Y}}\left(\iota_{\mathbf{V}}\left(g_{1}\right), \iota_{\mathbf{V}}\left(g_{2}\right)\right)=\beta_{\mathbf{V}, \xi}\left(g_{1} g_{2}\right) \beta_{\mathbf{V}, \xi}\left(g_{1}\right)^{-1} \beta_{\mathbf{V}, \xi}\left(g_{2}\right)^{-1} .
$$


In other words, with respect to the isomorphism $\mathrm{Mp}\left(\mathbf{V} \otimes_{E} \mathbf{W}\right) \cong \operatorname{Sp}\left(\mathbf{V} \otimes_{E} \mathbf{W}\right) \times \mathbb{C}^{1}$ determined by $z_{\mathbb{Y}}$, the following diagram commutes:

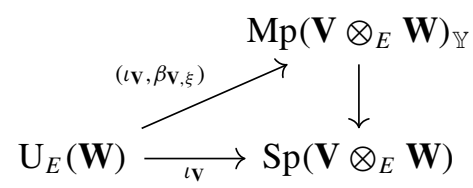

\subsection{Changing polarizations.}

Lemma 5.3 (Kudla, [K94, Lemma 4.2]). Let $\mathbb{X}+\mathbb{Y}$ and $\mathbb{X}^{\prime}+\mathbb{Y}^{\prime}$ be two polarizations of a symplectic space $\mathbb{V}$. Then

$$
z_{\mathbb{Y}^{\prime}}\left(g_{1}, g_{2}\right)=\lambda\left(g_{1} g_{2}\right) \lambda\left(g_{1}\right)^{-1} \lambda\left(g_{2}\right)^{-1} \cdot z_{\mathbb{Y}}\left(g_{1}, g_{2}\right),
$$

where $\lambda: \operatorname{Sp}(\mathbb{V}) \rightarrow \mathbb{C}^{1}$ is given by a product of Weil indices of Leray invariants:

$$
\lambda(g):=\lambda_{\mathbb{Y} \rightsquigarrow \mathbb{Y}^{\prime}}(g):=\gamma_{F}\left(\frac{1}{2} \psi \circ q\left(\mathbb{Y}, \mathbb{Y}^{\prime} g^{-1}, \mathbb{Y}^{\prime}\right)\right) \cdot \gamma_{F}\left(\frac{1}{2} \psi \circ q\left(\mathbb{Y}, \mathbb{Y}^{\prime}, \mathbb{Y} g\right)\right) .
$$

In particular, the bijection

$$
\operatorname{Mp}(\mathbb{V})_{\mathbb{Y}} \rightarrow \operatorname{Mp}(\mathbb{V})_{\mathbb{Y}^{\prime}}, \quad(g, z) \mapsto(g, z \cdot \lambda(g))
$$

is an isomorphism.

5.3. Three seesaws of unitary groups. For any two unitary similitude groups $\mathrm{GU}_{E}(\mathbf{V})$ and $\mathrm{GU}_{E}(\mathbf{W})$, we write

$$
\mathrm{G}\left(\mathrm{U}_{E}(\mathbf{V}) \times \mathrm{U}_{E}(\mathbf{W})\right):=\left\{(g, h) \in \mathrm{GU}_{E}(\mathbf{V}) \times \mathrm{GU}_{E}(\mathbf{W}): v(g)=v(h)\right\} .
$$

Fix a complete polarization $\mathbb{V}=\mathbb{X}+\mathbb{Y}$. In this section, we define splittings (of $z_{\mathbb{Y}}$ or $z_{\mathbb{Y}} \square$, depending on context) for the unitary groups $\mathrm{G}\left(\mathrm{U}_{E}\left(V_{0}^{\square}\right) \times \mathrm{U}_{E}\left(W_{0}\right)\right)$, $\mathrm{G}\left(\mathrm{U}_{E}\left(V_{0}\right) \times \mathrm{U}_{E}\left(W_{0}\right)\right), \mathrm{G}\left(\mathrm{U}_{E}(\operatorname{Res} V) \times \mathrm{U}_{E}\left(W^{\square}\right)\right)$, and $\mathrm{G}\left(\mathrm{U}_{E}(\operatorname{Res} V) \times \mathrm{U}_{E}(W)\right)$, which fit into the seesaw

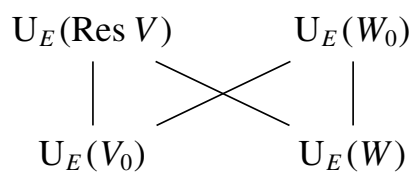

and the two corresponding doubling seesaws:

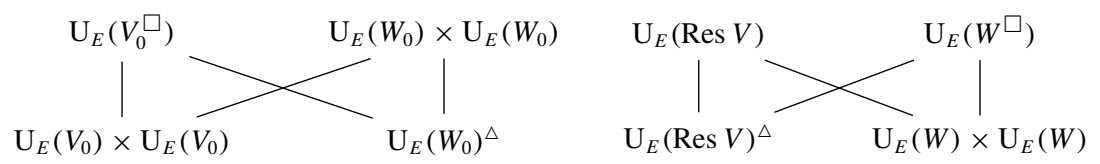


5.3.1. Splittings for $\mathrm{G}\left(\mathrm{U}_{E}\left(V_{0}^{\square}\right) \times \mathrm{U}_{E}\left(W_{0}\right)\right)$ and $\mathrm{G}\left(\mathrm{U}_{E}\left(V_{0}\right) \times \mathrm{U}_{E}\left(W_{0}\right)\right)$. Consider the 2-dimensional $E$-space $V_{0} \otimes_{E} W_{0}$ with skew-Hermitian form given by $\overline{(\cdot, \cdot)} \otimes$ $\langle\cdot, \cdot\rangle$. By a straightforward computation, we see that this allows us to identify $V_{0} \otimes_{E} W_{0}=W_{0}$ as $E$-spaces endowed with skew-Hermitian forms. Define

$$
\begin{aligned}
i: \mathrm{G}\left(\mathrm{U}_{E}\left(V_{0}\right) \times \mathrm{U}_{E}\left(W_{0}\right)\right) & \rightarrow \mathrm{U}_{E}\left(\left(V_{0} \otimes W_{0}\right)^{\square}\right), \\
(g, h) & \mapsto\left(\left(v \otimes w, v^{-} \otimes w^{-}\right) \mapsto\left(g^{-1} v \otimes w h, v^{-} \otimes w^{-}\right)\right), \\
i^{-}: \mathrm{G}\left(\mathrm{U}_{E}\left(V_{0}\right) \times \mathrm{U}_{E}\left(W_{0}\right)\right) & \rightarrow \mathrm{U}_{E}\left(\left(V_{0} \otimes W_{0}\right)^{\square}\right), \\
(g, h) & \mapsto\left(\left(v \otimes w, v^{-} \otimes w^{-}\right) \mapsto\left(v \otimes w, g^{-1} v^{-} \otimes w^{-} h\right)\right), \\
i^{\square}: \mathrm{G}\left(\mathrm{U}_{E}\left(V_{0}^{\square}\right) \times \mathrm{U}_{E}\left(W_{0}\right)\right) & \rightarrow \mathrm{U}_{E}\left(V_{0}^{\square} \otimes W_{0}\right), \\
(g, h) & \mapsto\left(v \otimes w \mapsto g^{-1} v \otimes w h\right) .
\end{aligned}
$$

We may identify $V_{0}^{\square} \otimes W_{0}=\left(V_{0} \otimes W_{0}\right)^{\square}=W_{0}^{\square}$. We have natural embeddings

$$
\begin{aligned}
\mathrm{G}\left(\mathrm{U}_{E}\left(V_{0}\right) \times \mathrm{U}_{E}\left(V_{0}\right) \times \mathrm{U}_{E}\left(W_{0}\right)\right) \hookrightarrow & \mathrm{G}\left(\mathrm{U}_{E}\left(V_{0}\right) \times \mathrm{U}_{E}\left(W_{0}\right)\right) \\
& \times \mathrm{G}\left(\mathrm{U}_{E}\left(V_{0}\right) \times \mathrm{U}_{E}\left(W_{0}\right)\right) \\
\mathrm{G}\left(\mathrm{U}_{E}\left(V_{0}\right) \times \mathrm{U}_{E}\left(V_{0}\right) \times \mathrm{U}_{E}\left(W_{0}\right)\right) \hookrightarrow & \mathrm{G}\left(\mathrm{U}_{E}\left(V_{0}^{\square}\right) \times \mathrm{U}_{E}\left(W_{0}\right)\right) .
\end{aligned}
$$

Observe that for $\left(g_{1}, g_{2}, h\right) \in \mathrm{G}\left(\mathrm{U}_{E}\left(V_{0}\right) \times \mathrm{U}_{E}\left(V_{0}\right) \times \mathrm{U}_{E}\left(W_{0}\right)\right)$,

$$
i\left(g_{1}, h\right) i^{-}\left(g_{2}, h\right)=i^{\square}\left(g_{1}, g_{2}, h\right) \in \mathrm{U}_{E}\left(W_{0}^{\square}\right) .
$$

We identify $\operatorname{Res}_{E / F}\left(W_{0}^{\square}\right)=\mathbb{V}^{\square}$ and let

$$
\iota: \mathrm{U}_{E}\left(W_{0}^{\square}\right) \rightarrow \operatorname{Sp}\left(\operatorname{Res}_{E / F}\left(W_{0}^{\square}\right)\right)=\operatorname{Sp}\left(\mathbb{V}^{\square}\right)
$$

be the natural embedding. We will often identify $\mathrm{U}_{E}\left(W_{0}^{\square}\right)$ with $\iota\left(\mathrm{U}_{E}\left(W_{0}^{\square}\right)\right)$.

DEFINITION 5.4. Pick a character $\xi: E^{\times} \rightarrow \mathbb{C}^{1}$ such that $\left.\xi\right|_{F^{\times}}=\epsilon_{E / F}$. Define

$$
\beta: \mathrm{U}_{E}\left(W_{0}^{\square}\right) \rightarrow \mathbb{C}^{1}, \quad g \mapsto \xi(x(g)) \cdot\left((u,-1)_{F} \gamma_{F}\left(u, \frac{1}{2} \psi\right)\right)^{-j(g)} .
$$

Define $\lambda:=\lambda_{V_{0} \otimes W_{0}^{\Delta} \rightsquigarrow \mathbb{Y} \square}: \operatorname{Sp}\left(\mathbb{V}^{\square}\right) \rightarrow \mathbb{C}^{1}$ and

$$
\begin{array}{lll}
\hat{s}:=i^{*} \beta, & \hat{s}^{-}:=\left(i^{-}\right)^{*} \beta, & \hat{s}^{\square}:=\left(i^{\square}\right)^{*} \beta, \\
s:=i^{*}(\beta \lambda), & s^{-}:=\left(i^{-}\right)^{*}(\beta \lambda), & s^{\square}:=\left(i^{\square}\right)^{*}(\beta \lambda) .
\end{array}
$$

LEMMA 5.5. (a) $\hat{s}, \hat{s}^{-}$, and $\hat{s}^{\square}$ are splittings of $z_{V_{0} \otimes W_{0}}$ on the images of $i, i^{-}$, and $i^{\square}$, respectively.

(b) $s$ is a splitting of $z_{\mathbb{Y}}$ on the image of $i, s^{-}$is a splitting of $z_{\mathbb{Y}}^{-1}$ on the image of $i^{-}$, and $s^{\square}$ is a splitting of $z_{\mathbb{Y}} \square$ on the image of $i^{\square}$. 
Proof. Observe that $\operatorname{det}\left(V_{0}\right)=1$ and $\operatorname{dim}\left(V_{0}\right)=1$ so that

$$
\gamma_{F}\left(\frac{1}{2} \psi \circ R V_{0}\right)=(u, 1)_{F} \gamma_{F}\left(-u, \frac{1}{2} \psi\right) \gamma_{F}\left(-1, \frac{1}{2} \psi\right)^{-1}=(u,-1)_{F} \gamma_{F}\left(u, \frac{1}{2} \psi\right) .
$$

This implies that $\beta=\beta_{\mathrm{U}_{E}\left(V_{0}\right), \xi}$ (see Definition 5.1) and hence is a splitting of $z_{V_{0} \otimes_{E} W_{0}^{\Delta}}$. Since $\hat{s}, \hat{s}^{-}$, and $\hat{s}^{\square}$ are pullbacks of $\beta$, they must also be splittings of the same cocycle.

LEMmA 5.6. For any $(g, h) \in \mathrm{G}\left(\mathrm{U}_{E}\left(V_{0}\right) \times \mathrm{U}_{E}\left(W_{0}\right)\right)$,

$$
\hat{s}^{-}(g, h)=\overline{\hat{s}(g, h)} \cdot \xi(\operatorname{det}(g, h)) .
$$

Proof. Let $d_{W_{0}^{\Delta}}(-1)=\left(\begin{array}{cc}1 & 0 \\ 0 & -1\end{array}\right)$ and set

$$
j_{W_{0}^{\Delta}}: \mathrm{U}_{E}\left(W_{0}^{\square}\right) \rightarrow \mathrm{U}_{E}\left(W_{0}^{\square}\right), \quad g \mapsto d_{W_{0}^{\Delta}}(-1) g d_{W_{0}^{\Delta}}(-1) .
$$

Let $g \in \mathrm{G}\left(\mathrm{U}_{E}\left(V_{0}\right) \times \mathrm{U}_{E}\left(W_{0}\right)\right)$. By a straightforward computation, we have

$$
x\left(i^{-}(g)\right)=(-1)^{j(g)} x(i(g)), \quad \text { and } \quad j\left(i^{-}(g)\right)=j(i(g)) .
$$

Therefore, since $\gamma_{F}\left(u, \frac{1}{2} \psi\right)^{2}=(u,-1)_{F}$,

$$
\begin{aligned}
\hat{s}^{-}(g) & =\xi\left(x\left(i^{-}(g)\right)\right)\left((u,-1)_{F} \gamma_{F}\left(u, \frac{1}{2} \psi\right)\right)^{-j\left(i^{-}(g)\right)} \\
& =\xi(x(i(g)))\left((u,-1)_{F} \gamma_{F}\left(u, \frac{1}{2} \psi\right)\right)^{j(i(g))} \\
& =\xi(x(i(g)))^{2} \overline{\hat{s}(g)}=\xi(\operatorname{det}(g)) \overline{\hat{s}(g)}
\end{aligned}
$$

LEMma 5.7. For $\left(g_{1}, g_{2}, h\right) \in \mathrm{G}\left(\mathrm{U}_{E}\left(V_{0}\right) \times \mathrm{U}_{E}\left(V_{0}\right) \times \mathrm{U}_{E}\left(W_{0}\right)\right)$,

$$
s^{\square}\left(g_{1}, g_{2}, h\right)=s\left(g_{1}, h\right) \cdot \overline{s\left(g_{2}, h\right)} \cdot \xi\left(\operatorname{det}\left(i\left(g_{2}, h\right)\right)\right) .
$$

Proof. This is [HKS96, Lemma 1.1]. See also [IP18++, Lemma D.4].

5.3.2. Splittings for $\mathrm{G}\left(\mathrm{U}_{E}(\operatorname{Res} V) \times \mathrm{U}_{E}\left(W^{\square}\right)\right)$ and $\mathrm{G}\left(\mathrm{U}_{E}(\operatorname{Res} V) \times \mathrm{U}_{E}(W)\right)$. This section is completely analogous to Section 5.3.1. The 2-dimensional $E$-space Res $V \otimes_{E} W$ with skew-Hermitian form $\overline{(\cdot, \cdot)} \otimes\langle\cdot, \cdot\rangle$ can be identified with Res $V$. Define

$$
\begin{aligned}
i^{\prime}: \mathrm{G}\left(\mathrm{U}_{E}(\operatorname{Res} V) \times \mathrm{U}_{E}(W)\right) & \rightarrow \mathrm{U}_{E}\left(\operatorname{Res} V^{\square}\right), & (g, h) \mapsto\left(\left(v, v^{-}\right) \mapsto\left(g^{-1} v h, v^{-}\right)\right), \\
i^{-\prime}: \mathrm{G}\left(\mathrm{U}_{E}(\operatorname{Res} V) \times \mathrm{U}_{E}(W)\right) & \rightarrow \mathrm{U}_{E}\left(\operatorname{Res} V^{\square}\right), & (g, h) \mapsto\left(\left(v, v^{-}\right) \mapsto\left(v, g^{-1} v^{-} h\right),\right. \\
i^{\square \prime}: \mathrm{G}\left(\mathrm{U}_{E}(\operatorname{Res} V) \times \mathrm{U}_{E}\left(W^{\square}\right)\right) & \rightarrow \mathrm{U}_{E}\left(\operatorname{Res} V^{\square}\right), & (g, h) \mapsto\left(v \mapsto g^{-1} v h\right) .
\end{aligned}
$$


We have natural embeddings

$$
\begin{aligned}
\mathrm{G}\left(\mathrm{U}_{E}(\operatorname{Res} V) \times \mathrm{U}_{E}(W) \times \mathrm{U}_{E}(W)\right) \hookrightarrow & \mathrm{G}\left(\mathrm{U}_{E}(\operatorname{Res} V) \times \mathrm{U}_{E}(W)\right) \\
& \times \mathrm{G}\left(\mathrm{U}_{E}(\operatorname{Res} V) \times \mathrm{U}_{E}(W)\right), \\
\mathrm{G}\left(\mathrm{U}_{E}(\operatorname{Res} V) \times \mathrm{U}_{E}(W) \times \mathrm{U}_{E}(W)\right) \hookrightarrow & \mathrm{G}\left(\mathrm{U}_{E}(\operatorname{Res} V) \times \mathrm{U}_{E}\left(W^{\square}\right)\right) .
\end{aligned}
$$

Observe that for $\left(g, h_{1}, h_{2}\right) \in \mathrm{G}\left(\mathrm{U}_{E}(\operatorname{Res} V) \times \mathrm{U}_{E}(W) \times \mathrm{U}_{E}(W)\right)$,

$$
i^{\prime}\left(g, h_{1}\right) i^{-\prime}\left(g, h_{2}\right)=i^{\square \prime}\left(g, h_{1}, h_{2}\right) \in \mathrm{U}_{E}\left(\operatorname{Res} V^{\square}\right) .
$$

We identify $\operatorname{Res}_{B / F}\left(V^{\square}\right)=\mathbb{V}^{\square}$ and let

$$
\iota^{\prime}: \mathrm{U}_{E}\left(\operatorname{Res} V^{\square}\right) \rightarrow \operatorname{Sp}\left(\mathbb{V}^{\square}\right)
$$

be the natural embedding. We will often identify $\mathrm{U}_{E}\left(\operatorname{Res} V^{\square}\right)$ with $\iota\left(\mathrm{U}_{E}\left(\operatorname{Res} V^{\square}\right)\right)$.

Definition 5.8. Pick a character $\xi^{\prime}: E^{\times} \rightarrow \mathbb{C}^{1}$ such that $\left.\xi^{\prime}\right|_{F^{\times}}=\epsilon_{E / F}$. Define

$$
\beta^{\prime}: \mathrm{U}_{E}\left(\operatorname{Res} V^{\square}\right) \rightarrow \mathbb{C}^{1}, \quad g \mapsto \xi^{\prime}(x(g)) \cdot\left((u,-1)_{F} \gamma_{F}\left(u, \frac{1}{2} \psi\right)\right)^{-j(g)} .
$$

Define

$$
\lambda^{\prime}:=\lambda_{\operatorname{Res} V \Delta \otimes W \rightsquigarrow \mathbb{Y} \square}: \operatorname{Sp}\left(\mathbb{V}^{\square}\right) \rightarrow \mathbb{C}^{1} .
$$

Define

$$
\begin{array}{lll}
\hat{s}^{\prime}:=\left(i^{\prime}\right)^{*} \beta^{\prime}, & \hat{s}^{-\prime}:=\left(i^{-\prime}\right)^{*} \beta^{\prime}, & \hat{s}^{\square \prime}:=\left(i^{\square \prime}\right)^{*} \beta^{\prime}, \\
s^{\prime}:=\left(i^{\prime}\right)^{*}\left(\beta^{\prime} \lambda^{\prime}\right), & s^{-\prime}:=\left(i^{-\prime}\right)^{*}\left(\beta^{\prime} \lambda^{\prime}\right), & s^{\square \prime}:=\left(i^{\square \prime}\right)^{*}\left(\beta^{\prime} \lambda^{\prime}\right) .
\end{array}
$$

LEMMA 5.9. (a) $\hat{s}^{\prime}, \hat{s}^{-\prime}$, and $\hat{s}^{\square \prime}$ are splittings of $z_{\operatorname{Res} V^{\Delta} \otimes W}$ on the images of $i^{\prime}$, $i^{-\prime}$, and $i^{\square \prime}$, respectively.

(b) $s^{\prime}$ is a splitting of $z_{\mathbb{Y}}$ on the image of $i^{\prime}, s^{-\prime}$ is a splitting of $z_{\mathbb{Y}}^{-1}$ on the image of $i^{-\prime}$, and $s^{\square \prime}$ is a splitting of $z_{\mathbb{Y}} \square$ on the image of $i^{\square \prime}$.

Lemma 5.10. For $\left(g, h_{1}, h_{2}\right) \in \mathrm{G}\left(\mathrm{U}_{E}(\operatorname{Res} V) \times \mathrm{U}_{E}(W) \times \mathrm{U}_{E}(W)\right)$,

$$
s^{\square \prime}\left(g, h_{1}, h_{2}\right)=s^{\prime}\left(g, h_{1}\right) \cdot \overline{s^{\prime}\left(g, h_{2}\right)} \cdot \xi^{\prime}\left(\operatorname{det}\left(i^{\prime}\left(g, h_{2}\right)\right)\right) .
$$

5.4. Compatibility between the splittings for the three seesaws. In this section, we investigate the compatibility of the splittings of the four pairs of unitary groups relative to the three seesaws presented in (5.1) and (5.2). Compatibility of the splittings in the two doubling seesaws of (5.2) is explicated 
in Lemmas 5.7 and 5.10. Hence it remains to investigate the compatibility of the splittings

$$
s: \mathrm{G}\left(\mathrm{U}_{E}\left(V_{0}\right) \times \mathrm{U}_{E}\left(W_{0}\right)\right) \rightarrow \mathbb{C}^{1} \text { and } s^{\prime}: \mathrm{G}\left(\mathrm{U}_{E}(\operatorname{Res} V) \times \mathrm{U}_{E}(W)\right) \rightarrow \mathbb{C}^{1} .
$$

Precisely, we would compare $s$ and $s^{\prime}$ on the subgroup

$$
\mathrm{G}\left(\mathrm{U}_{E}\left(V_{0}\right) \times \mathrm{U}_{E}(W)\right) \cong\left\{(\alpha, \beta) \in E^{\times} \times E^{\times}: \mathrm{Nm}(\alpha)=\mathrm{Nm}(\beta)\right\} .
$$

We prove a sequence of lemmas that break up the computation showing Proposition 5.14.

Let $\alpha, \beta \in E^{\times}$with $\mathrm{Nm}(\alpha)=\mathrm{Nm}(\beta)$ so that $(\alpha, \beta) \in \mathrm{G}\left(\mathrm{U}_{E}\left(V_{0}\right) \times \mathrm{U}_{E}(W)\right)$. Let $g \in \mathrm{U}_{E}\left(W_{0}^{\square}\right)$ denote the map $\left(w, w^{-}\right) \mapsto\left(\alpha^{-1} w \beta, w^{-}\right)$and let $g^{\prime} \in \mathrm{U}_{E}\left(\operatorname{Res} V^{\square}\right)$ denote the map $\left(v, v^{-}\right) \mapsto\left(\alpha^{-1} v \beta, v^{-}\right)$. Define

$$
v_{1}:=\left(-\frac{\mathbf{i}}{2 u}, \frac{\mathbf{i}}{2 u}\right), \quad v_{2}:=\left(\frac{\mathbf{i j}}{2 u J},-\frac{\mathbf{i j}}{2 u J}\right), \quad v_{1}^{\prime}:=(1,1), \quad v_{2}^{\prime}:=(\mathbf{j}, \mathbf{j}) .
$$

This defines an $E$-basis of $W_{0}^{\square}$ and of Res $V^{\square}$ with the following property:

$$
\begin{array}{cl}
\left(v_{i}, v_{j}^{\prime}\right)_{0}=\delta_{i j}, & \left(v_{i}, v_{j}\right)_{0}=\left(v_{i}^{\prime}, v_{j}^{\prime}\right)_{0}=0, \\
\left\langle v_{i}, v_{j}^{\prime}\right\rangle=\delta_{i j}, & \left\langle v_{i}, v_{j}\right\rangle=\left\langle v_{i}^{\prime}, v_{j}^{\prime}\right\rangle=0 .
\end{array}
$$

With respect to the basis $\left\{v_{1}, v_{2}, v_{1}^{\prime}, v_{2}^{\prime}\right\}$,

$$
g=\left(\begin{array}{cccc}
\frac{1+\alpha^{-1} \beta}{2} & 0 & \frac{1-\alpha^{-1} \beta}{4 u} \mathbf{i} & 0 \\
0 & \frac{1+\alpha^{-1} \bar{\beta}}{2} & 0 & -\frac{1-\alpha^{-1} \bar{\beta}}{4 u J} \mathbf{i} \\
\left(1-\alpha^{-1} \beta\right) \mathbf{i} & 0 & \frac{1+\alpha^{-1} \beta}{2} & 0 \\
0 & -\left(1-\alpha^{-1} \bar{\beta}\right) \mathbf{i} J & 0 & \frac{1+\alpha^{-1} \bar{\beta}}{2}
\end{array}\right) .
$$


Here, we view each unitary group as a subgroup of $\mathrm{GL}_{4}(E)$ with $\mathrm{GL}_{4}(E)$ acting formally by right multiplication. Note however that $W_{0}^{\square}$ is a left $E$-space, and so we interpret the formal multiplication $v \cdot a$ for $v \in W_{0}^{\square}$ and $a \in E$ as $a v$. Throughout this section, we write $\mathbf{g}$ when we want to refer to one of $g$ or $g^{\prime}$ simultaneously.

\section{LEMMA 5.11. We have}

\begin{tabular}{|c|c|c|c|}
\hline Conditions & $x(g)$ & $x\left(g^{\prime}\right)$ & $j(\mathbf{g})$ \\
\hline$\alpha^{-1} \beta=1, \alpha^{-1} \bar{\beta}=1$ & 1 & 1 & 0 \\
\hline$\alpha^{-1} \beta=1, \alpha^{-1} \bar{\beta} \neq 1$ & $-\left(1-\alpha^{-1} \bar{\beta}\right) \mathbf{i} J$ & $\left(1-\bar{\alpha}^{-1} \beta\right) \mathbf{i} J$ & 1 \\
\hline$\alpha^{-1} \beta \neq 1, \alpha^{-1} \bar{\beta}=1$ & $\left(1-\alpha^{-1} \beta\right) \mathbf{i}$ & $\left(1-\alpha^{-1} \beta\right) \mathbf{i}$ & 1 \\
\hline$\alpha^{-1} \beta \neq 1, \alpha^{-1} \bar{\beta} \neq 1$ & $-\left(1-\alpha^{-1} \beta\right)\left(1-\alpha^{-1} \bar{\beta}\right) u J$ & $\left(1-\alpha^{-1} \beta\right)\left(1-\bar{\alpha}^{-1} \beta\right) u J$ & 2 \\
\hline
\end{tabular}

Proof. The proof amounts to giving explicit decompositions

$$
\mathbf{g}=p_{1} w p_{2}, \quad \text { where } p_{i} \in P_{\mathbf{V}} \text { and } w=\tau_{j}=\left(\begin{array}{cc}
1_{2-j} & \\
& 1_{2-j}
\end{array}\right.
$$

There are four cases:

(a) If $\alpha^{-1} \beta=1$ and $\alpha^{-1} \bar{\beta}=1$, then

$$
g=1, \quad g^{\prime}=1 .
$$

(b) If $\alpha^{-1} \beta=1$ and $\alpha^{-1} \bar{\beta} \neq 1$, then $g=p_{1} \tau_{1} p_{2}$ and $g^{\prime}=p_{1}^{\prime} \tau_{1} p_{2}^{\prime}$ for

$$
\begin{gathered}
p_{1}=\left(\begin{array}{ccccc}
1 & 0 & 0 & 0 \\
0 & 1 & 0 & \frac{1+\alpha^{-1} \bar{\beta}}{2\left(-1+\alpha^{-1} \bar{\beta}\right) \mathbf{i} J} \\
0 & 0 & 1 & 0 \\
0 & 0 & 0 & 1
\end{array}\right), \\
p_{2}=\left(\begin{array}{cccc}
1 & 0 & 0 & 0 \\
0 & \left(-1+\alpha^{-1} \bar{\beta}\right) \mathbf{i} J & 0 & \frac{1+\alpha^{-1} \bar{\beta}}{2} \\
0 & 0 & 1 & 0 \\
0 & 0 & 0 & \frac{\alpha^{-1} \bar{\beta}}{\left(-1+\alpha^{-1} \bar{\beta}\right) \mathbf{i} J}
\end{array}\right),
\end{gathered}
$$




$$
\begin{gathered}
p_{1}^{\prime}=\left(\begin{array}{cccc}
1 & 0 & 0 & 0 \\
0 & 1 & 0 & -\frac{1+\bar{\alpha}^{-1} \beta}{2\left(-1+\bar{\alpha}^{-1} \beta\right) \mathbf{i} J} \\
0 & 0 & 1 & 0 \\
0 & 0 & 0 & 1
\end{array}\right), \\
p_{2}^{\prime}=\left(\begin{array}{cccc}
1 & 0 & 0 & 0 \\
0 & -\left(-1+\bar{\alpha}^{-1} \beta\right) \mathbf{i} J & 0 & \frac{1+\bar{\alpha}^{-1} \beta}{2} \\
0 & 0 & 1 & 0 \\
0 & 0 & 0 & -\frac{\bar{\alpha}^{-1} \beta}{\left(-1+\bar{\alpha}^{-1} \beta\right) \mathbf{i} J}
\end{array}\right) .
\end{gathered}
$$

(c) If $\alpha^{-1} \beta \neq 1$ and $\alpha^{-1} \bar{\beta}=1$, then

$$
g=g^{\prime}=\left(\begin{array}{cccc}
0 & 1 & 0 & \frac{1+\alpha^{-1} \beta}{2 \mathbf{i}\left(1-\alpha^{-1} \beta\right)} \\
1 & 0 & 0 & 0 \\
0 & 0 & 0 & 1 \\
0 & 0 & 1 & 0
\end{array}\right) \cdot \tau_{1} \cdot\left(\begin{array}{cccc}
0 & 1 & 0 & 0 \\
\left(1-\alpha^{-1} \beta\right) \mathbf{i} & 0 & \frac{1+\alpha^{-1} \beta}{2} & 0 \\
0 & 0 & 0 & 1 \\
0 & 0 & \frac{\alpha^{-1} \beta}{\left(1-\alpha^{-1} \beta\right) \mathbf{i}} & 0
\end{array}\right) .
$$

(d) If $\alpha^{-1} \beta \neq 1$ and $\alpha^{-1} \bar{\beta} \neq 1$, then $g=p_{1} \tau_{2} p_{2}$ and $g^{\prime}=p_{1}^{\prime} \tau_{2} p_{2}^{\prime}$ for

$$
\begin{aligned}
& p_{1}=\left(\begin{array}{cccc}
1 & 0 & \frac{1+\alpha^{-1} \beta}{2\left(1-\alpha^{-1} \beta\right) \mathbf{i}} & 0 \\
0 & 1 & 0 & -\frac{1+\alpha^{-1} \bar{\beta}}{2\left(1-\alpha^{-1} \bar{\beta}\right) \mathbf{i} J} \\
0 & 0 & 1 & 0 \\
0 & 0 & 0 & 1
\end{array}\right), \\
& p_{2}=\left(\begin{array}{cccc}
\left(1-\alpha^{-1} \beta\right) \mathbf{i} & 0 & \frac{1+\alpha^{-1} \beta}{2} & 0 \\
0 & -\left(1-\alpha^{-1} \bar{\beta}\right) \mathbf{i} J & 0 & \frac{1+\alpha^{-1} \bar{\beta}}{2} \\
0 & 0 & \frac{\alpha^{-1} \beta}{\left(1-\alpha^{-1} \beta\right) \mathbf{i}} & 0 \\
0 & 0 & 0 & -\frac{\alpha^{-1} \bar{\beta}}{\left(1-\alpha^{-1} \bar{\beta}\right) \mathbf{i} J}
\end{array}\right) \text {, }
\end{aligned}
$$




$$
\begin{aligned}
& p_{1}^{\prime}=\left(\begin{array}{cccc}
1 & 0 & \frac{1+\alpha^{-1} \beta}{2\left(1-\alpha^{-1} \beta\right) \mathbf{i}} & 0 \\
0 & 1 & 0 & \frac{1+\bar{\alpha}^{-1} \beta}{2\left(1-\bar{\alpha}^{-1} \beta\right) \mathbf{i} J} \\
0 & 0 & 1 & 0 \\
0 & 0 & 0 & 1
\end{array}\right), \\
& p_{2}^{\prime}=\left(\begin{array}{cccc}
\left(1-\alpha^{-1} \beta\right) \mathbf{i} & 0 & \frac{1+\alpha^{-1} \beta}{2} & 0 \\
0 & \left(1-\bar{\alpha}^{-1} \beta\right) \mathbf{i} J & 0 & \frac{1+\bar{\alpha}^{-1} \beta}{2} \\
0 & 0 & \frac{\alpha^{-1} \beta}{\left(1-\alpha^{-1} \beta\right) \mathbf{i}} & 0 \\
0 & 0 & 0 & \frac{\bar{\alpha}^{-1} \beta}{\left(1-\bar{\alpha}^{-1} \beta\right) \mathbf{i} J}
\end{array}\right) .
\end{aligned}
$$

From the above decompositions, we can easily read off the desired information.

LEMMA 5.12. Let $\alpha=a_{1}+b_{1}$ i. Then

$$
\begin{gathered}
\hat{s}(\alpha, \alpha)= \begin{cases}\xi\left(\alpha^{-1}\right) \cdot\left(a_{1}, u\right)_{F} & \text { if } b_{1}=0 \\
\xi\left(\alpha^{-1}\right) \cdot\left(-2 b_{1} u J, u\right)_{F} \cdot \gamma_{F}\left(u, \frac{1}{2} \psi\right) \cdot(-1,-u)_{F} & \text { otherwise. }\end{cases} \\
\hat{s}^{\prime}(\alpha, \alpha)= \begin{cases}\xi^{\prime}\left(\bar{\alpha}^{-1}\right) \cdot\left(a_{1}, u\right)_{F} & \text { if } b_{1}=0 \\
\xi^{\prime}\left(\bar{\alpha}^{-1}\right) \cdot\left(-2 b_{1} u J, u\right)_{F} \cdot \gamma_{F}\left(u, \frac{1}{2} \psi\right) \cdot(-1,-u)_{F} & \text { otherwise. }\end{cases}
\end{gathered}
$$

Proof. We use Lemma 5.11 in the two cases where $\alpha^{-1} \beta=1$. If $\alpha^{-1} \bar{\alpha}=1$, then $\alpha=\bar{\alpha}$ and so $b_{1}=0$. By Lemma 5.11, we have

$$
\hat{s}(\alpha, \alpha)=\hat{s}^{\prime}(\alpha, \alpha)=1=\xi\left(\alpha^{-1}\right) \cdot\left(a_{1}, u\right)_{F}=\xi^{\prime}\left(\alpha^{-1}\right) \cdot\left(a_{1}, u\right)_{F} .
$$

If $\alpha^{-1} \bar{\alpha} \neq 1$, then $b_{1} \neq 0$. Note that

$$
1-\alpha^{-1} \bar{\alpha}=\alpha^{-1}(\alpha-\bar{\alpha})=\alpha^{-1} \cdot 2 b_{1} \mathbf{i}, \quad 1-\bar{\alpha}^{-1} \alpha=\overline{1-\alpha^{-1} \bar{\alpha}}=-\bar{\alpha}^{-1} \cdot 2 b_{1} \mathbf{i} .
$$

The desired conclusion now follows by Lemma 5.11 . 
LEMMA 5.13. Let $\zeta=a+b \mathbf{i} \in E^{1}$. Then

$$
\begin{aligned}
& \hat{s}(1, \zeta)= \begin{cases}1 & \text { if } a=1, \\
((2-2 a) u J, u)_{F} & \text { if } a \neq 1,\end{cases} \\
& \hat{s}^{\prime}(1, \zeta)= \begin{cases}1 & \text { if } a=1, \\
\xi^{\prime}(\zeta) \cdot((2-2 a) u J, u)_{F} & \text { if } a \neq 1 .\end{cases}
\end{aligned}
$$

Proof. We use Lemma 5.11. If $\zeta=1$, this corresponds to the case $\alpha^{-1} \beta=1$, $\alpha^{-1} \bar{\beta}=1$, and

$$
\hat{s}(1, \zeta)=\hat{s}^{\prime}(1, \zeta)=1
$$

If $\zeta \neq 1$, this corresponds to the case $\alpha^{-1} \beta \neq 1, \alpha^{-1} \bar{\beta} \neq 1$, and $\hat{s}(1, \zeta)=\xi(-(1-\zeta)(1-\bar{\zeta}) u J) \cdot(-1, u)_{F}, \quad \hat{s}^{\prime}(1, \zeta)=\xi^{\prime}\left((1-\zeta)^{2} u J\right) \cdot(-1, u)_{F}$.

The desired conclusion follows from the simple observation

$$
(1-\zeta)(1-\bar{\zeta})=2-2 a, \quad(1-\zeta)^{2}=-\zeta(1-\zeta)(1-\bar{\zeta})=-\zeta(2-2 a)
$$

Proposition 5.14. Let $g \in \mathrm{G}\left(\mathrm{U}_{E}\left(V_{0}\right) \times \mathrm{U}_{E}(W)\right) \subset \mathrm{G}\left(\mathrm{U}_{E}\left(V_{0}\right) \times \mathrm{U}_{E}\left(W_{0}\right)\right)$ and $g^{\prime} \in \mathrm{G}\left(\mathrm{U}_{E}\left(V_{0}\right) \times \mathrm{U}_{E}(W)\right) \subset \mathrm{G}\left(\mathrm{U}_{E}(\operatorname{Res} V) \times \mathrm{U}_{E}(W)\right)$ correspond to $(\alpha, \beta) \in$ $E^{\times} \times E^{\times}$with $\mathrm{Nm}(\alpha)=\mathrm{Nm}(\beta)$. Then

$$
s^{\prime}\left(g^{\prime}\right)=\xi(\alpha) \xi^{\prime}(\beta) s(g) .
$$

Proof. We use the formulas given in Lemmas 5.12 and 5.13 together with Lemma 5.3. Recall that $g=g_{1} \cdot g_{2}, g^{\prime}=g_{1}^{\prime} \cdot g_{2}^{\prime}$, where $\mathbf{g}_{1}$ corresponds to $(\alpha, \alpha)$ and $\mathbf{g}_{2}$ corresponds to $(1, \beta / \alpha)$.

First note that under the natural maps

$$
\begin{array}{ll}
i: \mathrm{U}_{E}\left(V_{0} \otimes W_{0}\right) \rightarrow \operatorname{Sp}(\mathbb{V}), & i^{\square}: \mathrm{U}_{E}\left(V_{0} \otimes W_{0}\right) \rightarrow \operatorname{Sp}\left(\mathbb{V}^{\square}\right), \\
i^{\prime}: \mathrm{U}_{E}(\operatorname{Res} V \otimes W) \rightarrow \operatorname{Sp}(\mathbb{V}), & i^{\square \prime}: \mathrm{U}_{E}(\operatorname{Res} V \otimes W) \rightarrow \operatorname{Sp}\left(\mathbb{V}^{\square}\right),
\end{array}
$$

we have

$$
i\left(g_{\bullet}\right)=i^{\prime}\left(g_{\bullet}^{\prime}\right) \in \operatorname{Sp}(\mathbb{V}), \quad i^{\square}\left(g_{\bullet}\right)=i^{\square \prime}\left(g_{\bullet}^{\prime}\right) \in \operatorname{Sp}\left(\mathbb{V}^{\square}\right),
$$

where $\mathbf{g}$. denotes any of $\mathbf{g}, \mathbf{g}_{1}, \mathbf{g}_{2}$. This implies that for $\lambda:=\lambda_{\mathbb{V} \triangle} \rightsquigarrow \mathbb{Y} \square$,

$$
\lambda\left(i^{\square}\left(g_{\bullet}\right)\right)=\lambda\left(i^{\square \prime}\left(g_{\bullet}^{\prime}\right)\right) \quad \text { and } \quad z_{\mathbb{Y}}\left(i\left(g_{1}\right), i\left(g_{2}\right)\right)=z_{\mathbb{Y}}\left(i^{\prime}\left(g_{1}^{\prime}\right), i^{\prime}\left(g_{2}^{\prime}\right)\right) .
$$

By definition,

$$
\begin{gathered}
s(\mathbf{g})=\hat{s}\left(\mathbf{g}_{1}\right) \cdot \mu\left(\mathbf{g}_{1}\right) \cdot \hat{s}\left(\mathbf{g}_{2}\right) \cdot \mu\left(\mathbf{g}_{2}\right) \cdot z_{\mathbb{Y}}\left(i\left(\mathbf{g}_{1}\right), i\left(\mathbf{g}_{2}\right)\right), \\
s^{\prime}(\mathbf{g})=\hat{s}^{\prime}\left(\mathbf{g}_{1}\right) \cdot \mu\left(\mathbf{g}_{1}\right) \cdot \hat{s}^{\prime}\left(\mathbf{g}_{2}\right) \cdot \mu\left(\mathbf{g}_{2}\right) \cdot z_{\mathbb{Y}}\left(i^{\prime}\left(\mathbf{g}_{1}\right), i^{\prime}\left(\mathbf{g}_{2}\right)\right) .
\end{gathered}
$$


Thus we have

$$
\chi(\alpha, \beta)=s(g) \cdot s^{\prime}\left(g^{\prime}\right)^{-1}=\hat{s}\left(g_{1}\right) \cdot \hat{s}\left(g_{2}\right) \cdot \hat{s}^{\prime}\left(g_{1}^{\prime}\right)^{-1} \cdot \hat{s}^{\prime}\left(g_{2}^{\prime}\right)^{-1} .
$$

Now we combine the results of Lemmas 5.12 and 5.13 to compute $\chi(\alpha, \beta)$. Using the fact

$$
\bar{\alpha}^{-1} \cdot \beta \cdot \alpha^{-1}=\bar{\beta}^{-1},
$$

in the calculation of $\hat{s}^{\prime}\left(g_{1}^{\prime}\right) \hat{s}^{\prime}\left(g_{2}^{\prime}\right)$ when $\alpha \neq \beta$, we have

$$
\begin{array}{r}
\hat{s}\left(g_{1}\right) \cdot \hat{s}\left(g_{2}\right)= \begin{cases}\xi\left(\alpha^{-1}\right) \cdot\left(a_{1}, u\right)_{F} & \alpha \in F^{\times}, \alpha=\beta \\
\xi\left(\alpha^{-1}\right) \cdot\left(a_{1}, u\right)_{F} \cdot((2-2 a) u J, u)_{F} & \alpha \in F^{\times}, \alpha \neq \beta \\
\xi\left(\alpha^{-1}\right) \cdot\left(-2 b_{1} u J, u\right)_{F} \cdot \gamma_{F}\left(u, \frac{1}{2} \psi\right) \cdot(-1,-u)_{F} & \alpha \notin F^{\times}, \alpha=\beta \\
\xi\left(\alpha^{-1}\right) \cdot\left(-2 b_{1} u J, u\right)_{F} \cdot \gamma_{F}\left(u, \frac{1}{2} \psi\right) \cdot(-1,-u)_{F} & \\
\cdot((2-2 a) u J, u)_{F} & \alpha \notin F^{\times}, \alpha \neq \beta\end{cases} \\
\hat{s}^{\prime}\left(g_{1}^{\prime}\right) \cdot \hat{s}^{\prime}\left(g_{2}^{\prime}\right)= \begin{cases}\xi^{\prime}\left(\bar{\alpha}^{-1}\right) \cdot\left(a_{1}, u\right)_{F} & \alpha \in F^{\times}, \alpha \neq \beta \\
\xi^{\prime}\left(\bar{\beta}^{-1}\right) \cdot\left(a_{1}, u\right)_{F} \cdot((2-2 a) u J, u)_{F} & \alpha \notin F, \alpha=\beta \\
\xi^{\prime}\left(\bar{\alpha}^{-1}\right) \cdot\left(-2 b_{1} u J, u\right)_{F} \cdot \gamma_{F}\left(u, \frac{1}{2} \psi\right) \cdot(-1,-u)_{F} & \alpha \notin F_{F} \\
\xi^{\prime}\left(\bar{\beta}^{-1}\right) \cdot\left(-2 b_{1} u J, u\right)_{F} \cdot \gamma_{F}\left(u, \frac{1}{2} \psi\right) \cdot(-1,-u)_{F} & \alpha \notin F^{\times}, \alpha \neq \beta . \\
\cdot((2-2 a) u J, u)_{F} & \end{cases}
\end{array}
$$

Therefore

$$
\begin{aligned}
\chi(\alpha, \beta) & = \begin{cases}\xi\left(\alpha^{-1}\right) \cdot \xi^{\prime}(\bar{\alpha}) & \alpha \in F^{\times}, \alpha=\beta \\
\xi\left(\alpha^{-1}\right) \cdot \xi^{\prime}(\bar{\beta}) & \alpha \in F^{\times}, \alpha \neq \beta \\
\xi\left(\alpha^{-1}\right) \cdot \xi^{\prime}(\bar{\alpha}) & \alpha \notin F^{\times}, \alpha=\beta \\
\xi\left(\alpha^{-1}\right) \cdot \xi^{\prime}(\bar{\beta}) & \alpha \notin F^{\times}, \alpha \neq \beta\end{cases} \\
& =\xi\left(\alpha^{-1}\right) \cdot \xi^{\prime}(\bar{\beta})=\xi\left(\alpha^{-1}\right) \cdot \xi^{\prime}\left(\beta^{-1}\right) \cdot \xi^{\prime}(\beta \bar{\beta})=\xi\left(\alpha^{-1}\right) \xi^{\prime}\left(\beta^{-1}\right) .
\end{aligned}
$$

5.5. Product formula. In this section, we put the local considerations of Sections 5.1-5.4 into the global picture. Once and for all, pick Hecke characters

$$
\xi, \xi^{\prime}: E^{\times} \backslash \mathbb{A}_{E}^{\times} \rightarrow \mathbb{C}^{1} \text { such that }\left.\xi\right|_{\mathbb{A}_{F}^{\times}}=\left.\xi^{\prime}\right|_{\mathbb{A}_{F}^{\times}}=\epsilon_{E / F} .
$$

Note that $\mathrm{U}_{E}\left(V_{0}\right) \cong E^{\times} \cong \mathrm{U}_{B}(V)^{0}$ and hence we have natural embeddings

$$
\begin{aligned}
\mathrm{G}\left(\mathrm{U}_{B}(V)^{0} \times \mathrm{U}_{B}(W)\right) & \hookrightarrow \mathrm{G}\left(\mathrm{U}_{E}\left(V_{0}\right) \times \mathrm{U}_{E}\left(W_{0}\right)\right) \\
\mathrm{G}\left(\mathrm{U}_{B}\left(V^{\square}\right)^{0} \times \mathrm{U}_{B}(W)\right) & \hookrightarrow \mathrm{G}\left(\mathrm{U}_{E}\left(V_{0}^{\square}\right) \times \mathrm{U}_{E}\left(W_{0}\right)\right) .
\end{aligned}
$$

Thus functions defined on the unitary spaces pull back to functions on the quaternionic unitary spaces. For each place $v$ of $F$, by Definitions 5.4 and 5.8, 
we have functions

$s_{v}: \mathrm{G}\left(\mathrm{U}_{B}\left(V_{v}\right) \times \mathrm{U}_{B}\left(W_{v}^{*}\right)\right) \rightarrow \mathbb{C}^{1}, \quad s_{v}^{\square}: \mathrm{G}\left(\mathrm{U}_{B}\left(V_{v}^{\square}\right)^{0} \times \mathrm{U}_{B}\left(W_{v}^{*}\right)\right) \rightarrow \mathbb{C}^{1}$,

$s_{v}^{\prime}: \mathrm{G}\left(\mathrm{U}_{E}\left(\operatorname{Res} V_{v}\right) \times \mathrm{U}_{E}\left(W_{v}\right)\right) \rightarrow \mathbb{C}^{1}, \quad s_{v}^{\square \prime}: \mathrm{G}\left(\mathrm{U}_{E}\left(\operatorname{Res} V_{v}\right) \times \mathrm{U}_{E}\left(W_{v}^{\square}\right)\right) \rightarrow \mathbb{C}^{1}$.

Formally define

$$
s:=\prod_{v} s_{v}, \quad s^{\prime}:=\prod_{v} s_{v}^{\prime}, \quad s^{\square}:=\prod_{v} s_{v}^{\square}, \quad s^{\prime \square}:=\prod_{v} s_{v}^{\prime \square} .
$$

These products converge by the following lemma, where we write 'a.a.' for 'all but finitely many'.

LEMMA 5.15. (a) Let $\gamma \in \mathrm{G}\left(\mathrm{U}_{B}(V)(F) \times \mathrm{U}_{B}(W)(F)\right)$. Then $s_{v}(\gamma)=1$ for a.a. $v$ and $s(\gamma)=1$.

(b) Let $\gamma \in \mathrm{G}\left(\mathrm{U}_{B}\left(V^{\square}\right)^{0}(F) \times \mathrm{U}_{B}(W)(F)\right)$. Then $s_{v}^{\square}(\gamma)=1$ for a.a. $v$ and $s^{\square}(\gamma)=1$.

(c) Let $\gamma \in \mathrm{G}\left(\mathrm{U}_{E}(\operatorname{Res} V)(F) \times \mathrm{U}_{E}(W)(F)\right)$. Then $s_{v}^{\prime}(\gamma)=1$ for a.a. $v$ and $s^{\prime}(\gamma)=1$.

(d) Let $\gamma \in \mathrm{G}\left(\mathrm{U}_{E}(\operatorname{Res} V)(F) \times \mathrm{U}_{E}\left(W^{\square}\right)(F)\right)$. Then $s_{v}^{\square \prime}(\gamma)=1$ for a.a. $v$ and $s^{\square \prime}(\gamma)=1$.

Proposition 5.16. (a) [Lemma 5.7] For $\left(g_{1}, g_{2}, h\right) \in \mathrm{G}\left(\mathrm{U}_{B}(V)^{0}(\mathbb{A}) \times\right.$ $\left.\mathrm{U}_{B}(V)^{0}(\mathbb{A}) \times \mathrm{U}_{B}(W)(\mathbb{A})\right)$,

$$
s^{\square}\left(g_{1}, g_{2}, h\right)=s\left(g_{1}, h\right) \cdot \overline{s\left(g_{2}, h\right)} \cdot \xi\left(\operatorname{det}\left(i\left(g_{2}, h\right)\right)\right) .
$$

(b) [Lemma 5.10] For $\left(h, g_{1}, g_{2}\right) \in \mathrm{G}\left(\mathrm{U}_{E}(\operatorname{Res} V)(\mathbb{A}) \times \mathrm{U}_{E}(W)(\mathbb{A}) \times\right.$ $\left.\mathrm{U}_{E}(W)(\mathbb{A})\right)$,

$$
s^{\square \prime}\left(h, g_{1}, g_{2}\right)=s^{\prime}\left(h, g_{1}\right) \cdot \overline{s^{\prime}\left(h, g_{2}\right)} \cdot \xi^{\prime}\left(\operatorname{det}\left(i^{\prime}\left(h, g_{2}\right)\right)\right) .
$$

(c) [Proposition 5.14] For $\alpha, \beta \in \mathbb{A}_{E}^{\times}$such that $\operatorname{Nm}(\alpha)=\operatorname{Nm}(\beta)$,

$$
s^{\prime}(\alpha, \beta)=\xi(\alpha) \xi^{\prime}(\beta) s(\alpha, \beta)
$$

5.6. Two splittings on $E_{v}^{\times} \times \mathbf{G L}_{2}\left(\boldsymbol{F}_{v}\right)$. To calculate the theta lift at all the unramified places, we will have to understand the Weil representation more concretely. In particular, we will need to explicate the local splittings defined in Section 5 in the cases $v \notin \Sigma_{B}$ and $v \notin \Sigma_{B^{\prime}}$. These exactly correspond, respectively, 
to the cases when $W_{0, v}$ and $\operatorname{Res} V_{v}$ are split Hermitian spaces. For notational convenience, we drop the subscript $v$ in this section.

Consider the group

$$
R:=\mathrm{G}\left(E^{\times} \times \mathrm{GL}_{2}(F)\right)=\left\{(\alpha, g) \in E^{\times} \times \mathrm{GL}_{2}(F): \mathrm{Nm}(\alpha)=\operatorname{det}(g)\right\} .
$$

Assume that the 2-dimensional $E$-spaces $W_{0}$ and $\operatorname{Res} V$ are hyperbolic planes (that is, they are split Hermitian spaces). Then we have embeddings

$$
\begin{aligned}
& R \hookrightarrow \mathrm{G}\left(\mathrm{U}_{E}\left(V_{0}\right) \times \mathrm{U}_{E}\left(W_{0}\right)\right), \quad(\alpha, g) \mapsto(\alpha, g) \\
& R \hookrightarrow \mathrm{G}\left(\mathrm{U}_{E}(\operatorname{Res} V) \times \mathrm{U}_{E}(W)\right), \quad(\alpha, g) \mapsto(g, \alpha) .
\end{aligned}
$$

Furthermore, any decomposition of $W_{0}$ or Res $V$ into maximal isotropic subspaces induces a complete polarization

$$
\mathbb{V}=\mathbb{X}^{\prime}+\mathbb{Y}^{\prime}
$$

Our goal in this section is to explicate the values of the splittings in Section 5.3 associated with this particular polarization. To make it clear that we are working in this specialized context, we let

$$
\text { s: } \mathrm{G}\left(\mathrm{U}_{E}\left(V_{0}\right) \times \mathrm{U}_{E}\left(W_{0}\right)\right) \rightarrow \mathbb{C}^{1}, \quad \mathbf{s}^{\prime}: \mathrm{G}\left(\mathrm{U}_{E}(\operatorname{Res} V) \times \mathrm{U}_{E}(W)\right) \rightarrow \mathbb{C}^{1}
$$

denote the splittings for $z_{\mathbb{Y}^{\prime}}$ defined in Section 5.3.

We briefly recall the construction of $\mathbf{s}, \mathbf{s}^{\prime}$. Recall that from Sections 5.3.1 and 5.3.2, we have natural maps

$$
\begin{aligned}
& i: \mathrm{G}\left(\mathrm{U}_{E}\left(V_{0}\right) \times \mathrm{U}_{E}\left(W_{0}\right)\right) \rightarrow \mathrm{U}_{E}\left(W_{0}^{\square}\right), \\
& i^{\prime}: \mathrm{G}\left(\mathrm{U}_{E}(\operatorname{Res} V) \times \mathrm{U}_{E}(W)\right) \rightarrow \mathrm{U}_{E}\left(\operatorname{Res} V^{\square}\right) .
\end{aligned}
$$

If we let $\lambda: \operatorname{Sp}\left(\mathbb{V}^{\square}\right) \rightarrow \mathbb{C}^{1}$ be given by

$$
\lambda(g):=\gamma_{F}\left(\frac{1}{2} \psi \circ q\left(\mathbb{V}^{\Delta}, \mathbb{Y}^{\prime} g^{-1}, \mathbb{Y}^{\prime \square}\right)\right) \cdot \gamma_{F}\left(\frac{1}{2} \circ q\left(\mathbb{V}^{\Delta}, \mathbb{Y}^{\prime \square}, \mathbb{V}^{\Delta} g\right)\right),
$$

then we have

$$
\begin{aligned}
& \mathbf{s}:=\hat{s} \cdot \lambda: \mathrm{G}\left(\mathrm{U}_{E}\left(V_{0}\right) \times \mathrm{U}_{E}\left(W_{0}\right)\right) \rightarrow \mathbb{C}^{1}, \\
& \mathbf{s}^{\prime}:=\hat{s}^{\prime} \cdot \lambda: \mathrm{G}\left(\mathrm{U}_{E}(\operatorname{Res} V) \times \mathrm{U}_{E}(W)\right) \rightarrow \mathbb{C}^{1},
\end{aligned}
$$

where $\partial \hat{s}=z_{V_{0} \otimes W_{0}^{\Delta}}$ and $\partial \hat{s}^{\prime}=z_{\operatorname{Res} V^{\Delta} \otimes W}$.

Let $W_{1}$ and $W_{2}$ be isotropic subspaces such that $W_{0}=W_{1}+W_{2}$ and fix $w_{i} \in W_{i}$ so that $\left\langle w_{1}, w_{2}\right\rangle=1$. Analogously, let $V_{1}$ and $V_{2}$ be isotropic subspaces such that $\operatorname{Res} V=V_{1}+V_{2}$ and fix $w_{i} \in V_{i}$ such that $\left\langle w_{1}, w_{2}\right\rangle=1$. Define

$$
\mathbf{w}_{1}=\left(\frac{1}{2} w_{1},-\frac{1}{2} w_{1}\right), \quad \mathbf{w}_{2}=\left(-\frac{1}{2} w_{2}, \frac{1}{2} w_{2}\right), \quad \mathbf{w}_{1}^{*}=\left(w_{2}, w_{2}\right), \quad \mathbf{w}_{2}^{*}=\left(w_{1}, w_{1}\right)
$$


so that we have $\left\langle\mathbf{w}_{i}, \mathbf{w}_{j}\right\rangle=\left\langle\mathbf{w}_{i}^{*}, \mathbf{w}_{j}^{*}\right\rangle,\left\langle\mathbf{w}_{i}, \mathbf{w}_{j}^{*}\right\rangle=\delta_{i j},\left\langle\mathbf{w}_{i}^{*}, \mathbf{w}_{j}\right\rangle=-\delta_{i j}$, and

$$
W_{0}^{\square}=W_{0}^{\nabla}+W_{0}^{\triangle}, \quad \text { where } W_{0}^{\nabla}=\operatorname{span}\left\{\mathbf{w}_{1}, \mathbf{w}_{2}\right\} \text { and } W_{0}^{\triangle}=\operatorname{span}\left\{\mathbf{w}_{1}^{*}, \mathbf{w}_{2}^{*}\right\},
$$

$\operatorname{Res} V^{\square}=\operatorname{Res} V^{\nabla}+\operatorname{Res} V^{\Delta}, \quad$ where Res $V^{\nabla}=\operatorname{span}\left\{\mathbf{w}_{1}, \mathbf{w}_{2}\right\}$

$$
\text { and } \operatorname{Res} V^{\triangle}=\operatorname{span}\left\{\mathbf{w}_{1}^{*}, \mathbf{w}_{2}^{*}\right\} \text {. }
$$

Then a symplectic basis preserving the complete polarization $\mathbb{V}^{\square}=\mathbb{V}^{\nabla}+\mathbb{V}^{\Delta}$ is given by

$$
\mathbf{w}_{1}, \quad \frac{-1}{u} \mathbf{i} \mathbf{w}_{1}, \quad \mathbf{w}_{2}, \quad \frac{-1}{u} \mathbf{i} \mathbf{w}_{2}, \quad \mathbf{w}_{1}^{*}, \quad \mathbf{i} \mathbf{w}_{1}^{*}, \quad \mathbf{w}_{2}^{*}, \quad \mathbf{i} \mathbf{w}_{2}^{*} .
$$

5.6.1. A splitting $\mathbf{s}$ of $z_{\mathbb{Y}^{\prime}}$. For $a, d \in F^{\times}$, write $D(a, d):=\operatorname{diag}(a, d)$.

LemMA 5.17. Let $(\alpha, D(a, d)) \in R$. Then

$$
\mathbf{s}(\alpha, D(a, d))=\xi\left(-\left(\alpha^{-1} a-1\right)\left(\alpha^{-1} d-1\right)\right) .
$$

In particular, for $a \in F^{\times}$and $\alpha \in E^{\times}$,

$$
\mathbf{s}\left(1, D\left(a, a^{-1}\right)\right)=(u, a)_{F}, \quad \mathbf{s}(\alpha, D(1, \mathrm{Nm}(\alpha)))=\xi\left(\alpha^{-1}\right) .
$$

Proof. We have $(1, D(1,1))=(1, U(0))$, and this is proved in Lemma 5.18, so we assume that $(\alpha, D(a, d)) \neq(1, D(1,1))$. This assumption will be necessary when we calculate $\hat{s}$.

Recall that $(\alpha, D(a, d))$ sends $w_{1} \mapsto \alpha^{-1} a w_{1}$ and $w_{2} \mapsto \alpha^{-1} d w_{2}$. Recalling that $i: \mathrm{U}_{E}\left(W_{0}\right) \rightarrow \mathrm{U}_{E}\left(W_{0}+W_{0}^{-}\right)$is defined by $\mathrm{U}_{E}\left(W_{0}\right)$ acting linearly on $W_{0}$ and trivially on $W_{0}^{-}$, it is a straightforward computation to see that the image of $(\alpha, D(a, d))$ in $\mathrm{U}_{E}\left(W_{0}+W_{0}^{-}\right)$with respect to the basis $\mathbf{w}_{1}, \mathbf{w}_{2}, \mathbf{w}_{1}^{*}, \mathbf{w}_{2}^{*}$ is

$$
\left(\begin{array}{cccc}
\frac{\alpha^{-1} a+1}{2} & 0 & 0 & \frac{\alpha^{-1} a-1}{4} \\
0 & \frac{\alpha^{-1} d+1}{2} & -\frac{\alpha^{-1} d-1}{4} & 0 \\
0 & -\left(\alpha^{-1} d-1\right) & \frac{\alpha^{-1} d+1}{2} & 0 \\
-\left(\alpha^{-1} a-1\right) & 0 & 0 & \frac{\alpha^{-1} a+1}{2}
\end{array}\right)
$$

We have

$$
i(\alpha, D(a, d))=p_{1}\left(\begin{array}{llll} 
& & 1 & \\
& & & 1 \\
-1 & & & \\
& -1 &
\end{array}\right) p_{2}
$$


where

$$
\begin{aligned}
& p_{1}=\left(\begin{array}{cccc}
0 & -\frac{\left(\alpha^{-1} a+1\right)^{2}}{4\left(\alpha^{-1} a-1\right)}+\frac{\alpha^{-1} a-1}{4} & -\frac{\alpha^{-1} a+1}{2} & 0 \\
\frac{\left(\alpha^{-1} d+1\right)^{2}}{4\left(\alpha^{-1} d-1\right)}-\frac{\alpha^{-1} d-1}{4} & 0 & 0 & -\frac{\alpha^{-1} d+1}{2} \\
0 & 0 & 0 & \left(\alpha^{-1} d-1\right) \\
0 & 0 & -\left(\alpha^{-1} a-1\right) & 0
\end{array}\right), \\
& p_{2}=\left(\begin{array}{cccc}
1 & 0 & 0 & \frac{\alpha^{-1} a+1}{2\left(\alpha^{-1} a-1\right)} \\
0 & 1 & -\frac{\alpha^{-1} d+1}{4\left(\alpha^{-1} d-1\right)} & 0 \\
0 & 0 & 1 & 0 \\
0 & 0 & 0 & 1
\end{array}\right)
\end{aligned}
$$

This implies that

$$
x(i(\alpha, D(a, d)))=\left(\alpha^{-1} a-1\right)\left(\alpha^{-1} d-1\right), \quad j(i(\alpha, D(a, d)))=2,
$$

and therefore by Definition 5.4,

$$
\begin{aligned}
\hat{s}(i(\alpha, D(a, d))) & =\xi\left(\left(\alpha^{-1} a-1\right)\left(\alpha^{-1} d-1\right)\right) \cdot \gamma_{F}\left(u, \frac{1}{2} \psi\right)^{-2} \\
& =\xi\left(-\left(\alpha^{-1} a-1\right)\left(\alpha^{-1} d-1\right)\right) .
\end{aligned}
$$

With respect to the symplectic basis given in (5.5), the image of $i(\alpha, D(a, d))$ in $\operatorname{Sp}\left(\mathbb{V}^{\square}\right)$ is

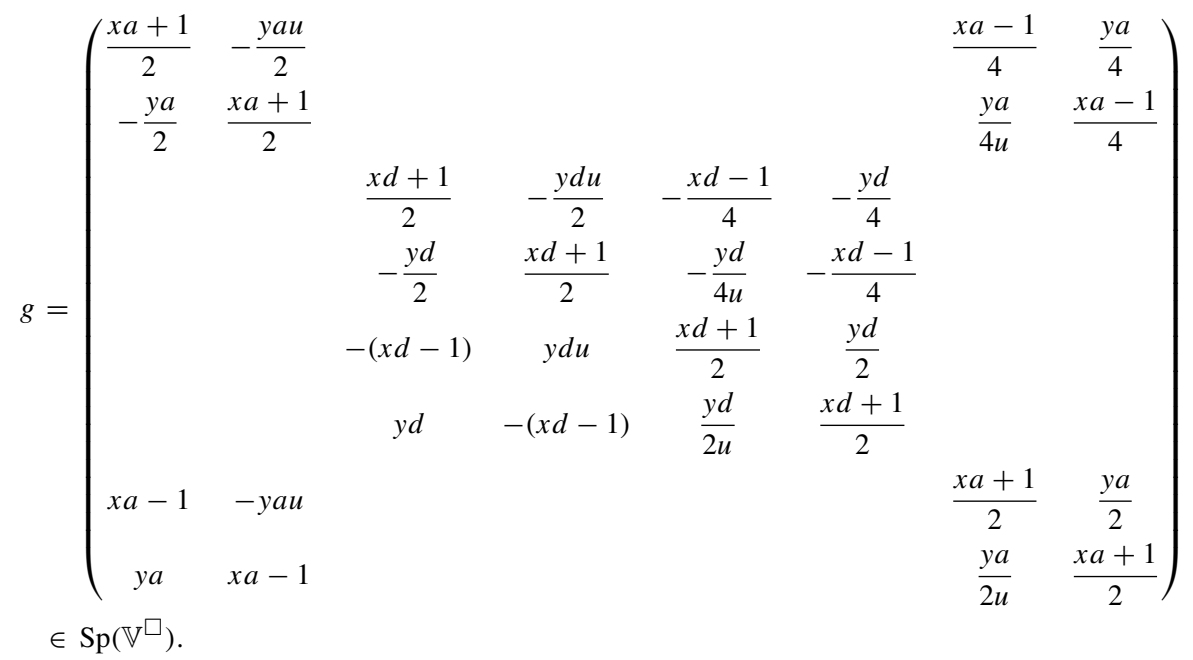


By definition,

$$
\lambda(\alpha, D(a, d))=\gamma_{F}\left(\frac{1}{2} \psi \circ q\left(\mathbb{V}^{\Delta}, \mathbb{Y}^{\square} g^{-1}, \mathbb{Y}^{\prime \square}\right)\right) \cdot \gamma_{F}\left(\frac{1}{2} \psi \circ q\left(\mathbb{V}^{\Delta}, \mathbb{Y}^{\prime \square}, \mathbb{V}^{\Delta} g\right)\right) .
$$

Since $g$ stabilizes $\mathbb{Y}^{\prime}$,

$$
\gamma_{F}\left(\frac{1}{2} \psi \circ q\left(\mathbb{V}^{\Delta}, \mathbb{Y}^{\prime \square} g^{-1}, \mathbb{Y}^{\prime \square}\right)\right)=1 .
$$

To calculate the second factor, note that

$$
\begin{aligned}
\mathbb{V}^{\Delta}= & \left\{\left(0,0,0,0, z_{1}, z_{2}, z_{3}, z_{4}\right)\right\}, \\
\mathbb{Y}^{\prime}= & \left\{\left(0,0, z_{1}, z_{2}, z_{3}, z_{4}, 0,0\right)\right\}, \\
\mathbb{V}^{\Delta} g= & \left\{\left((x a-1) z_{2}+y a z_{4},-y a u z_{3}+(x a-1) z_{4},\right.\right. \\
& -(x d-1) z_{1}+y d z_{2}, y d u z_{1}-(x d-1) z_{2},-\frac{x d+1}{2} z_{1}-\frac{y d}{2 u} z_{2}, \\
& \left.\left.-\frac{y d}{2} z_{1}-\frac{x d+1}{2} z_{2}, \frac{x a+1}{2} z_{3}+\frac{y a}{2 u} z_{4}, \frac{y a}{2} z_{3}+\frac{x a+1}{2} z_{4}\right)\right\},
\end{aligned}
$$

and one can see that this implies that $\mathbb{R}:=\mathbb{V}^{\Delta} \cap \mathbb{Y}^{\square}+\mathbb{V}^{\Delta} \cap \mathbb{V}^{\Delta} g+\mathbb{Y}^{\prime} \cap \mathbb{V}^{\Delta} g=$ $\{(0,0, *, *, *, *, 0,0)\}$ and hence

$$
\gamma_{F}\left(\frac{1}{2} \psi \circ q\left(\mathbb{V}^{\Delta}, \mathbb{Y}^{\prime \square}, \mathbb{V}^{\Delta} g\right)\right)=1 .
$$

We therefore have

$$
\mathbf{s}(\alpha, D(a, d))=\hat{s}(\alpha, D(a, d))=\xi\left(-\left(\alpha^{-1} a-1\right)\left(\alpha^{-1} d-1\right)\right) .
$$

This proves the main assertion and the remaining formulas can be deduced as follows: assuming $a \neq 1$ and $\alpha \neq 1$ (observe that if $\alpha \in E^{1}$, then $x=1$ if and only if $\alpha=1$ ),

$\mathbf{s}\left(1, D\left(a, a^{-1}\right)\right)=\xi\left(-(a-1)\left(a^{-1}-1\right)\right)=\xi\left(a^{-1}(a-1)^{2}\right)=\xi\left(a^{-1}\right)=(u, a)_{F}$.

If $\alpha \in E^{\times}$, then

$$
\begin{aligned}
\mathbf{s}(\alpha, D(1, \mathrm{Nm}(\alpha))) & =\xi\left(-\left(\alpha^{-1}-1\right)\left(\alpha^{-1} \alpha \bar{\alpha}-1\right)\right) \\
& =\xi\left(\alpha^{-1}\right) \epsilon_{E / F}(\mathrm{Nm}(\alpha-1))=\xi\left(\alpha^{-1}\right) .
\end{aligned}
$$

Lemma 5.18. Let $a \in F$. Then

$$
\mathbf{s}(1, U(a))=1 .
$$


Proof. The matrix $U(a)$ sends $w_{1} \mapsto w_{1}+a w_{2}$ and $w_{2} \mapsto w_{2}$. Recalling that $i: \mathrm{U}_{E}\left(W_{0}\right) \rightarrow \mathrm{U}_{E}\left(W_{0}+W_{0}^{-}\right)$is defined by $\mathrm{U}_{E}\left(W_{0}\right)$ acting linearly on $W_{0}$ and trivially on $W_{0}^{-}$, it is a straightforward computation to see that

$$
i(1, U(a))=\left(\begin{array}{cccc}
1 & -\frac{a}{2} & \frac{a}{4} & 0 \\
0 & 1 & 0 & 0 \\
0 & 0 & 1 & 0 \\
0 & -a & \frac{a}{2} & 1
\end{array}\right)
$$

We have

$$
\left(\begin{array}{cccc}
1 & -\frac{a}{2} & \frac{a}{4} & 0 \\
0 & 1 & 0 & 0 \\
0 & 0 & 1 & 0 \\
0 & -a & \frac{a}{2} & 1
\end{array}\right)\left(\begin{array}{ccccc}
1 & 0 & 0 & 0 \\
0 & a^{-1} & 0 & 1 \\
0 & 0 & 1 & 0 \\
0 & 0 & 0 & a
\end{array}\right)=\left(\begin{array}{cccc}
1 & \frac{a}{2} & \frac{a}{4} & -\frac{1}{2} \\
0 & -1 & 0 & a^{-1} \\
0 & 0 & 1 & 0 \\
0 & 0 & \frac{a}{2} & -1
\end{array}\right)\left(\begin{array}{llll}
1 & & \\
& & & -1 \\
& 1 & &
\end{array}\right),
$$

and therefore $x(i(1, U(a)))=-a^{-1}$ and $j(i(1, U(a)))=1$. By Definition 5.4, we have

$$
\hat{s}(1, U(a))= \begin{cases}1 & \text { if } a=0, \\ \xi\left(-a^{-1}\right) \cdot(u,-1)_{F} & \\ \cdot \gamma_{F}\left(u, \frac{1}{2} \psi\right)^{-1}=(u, a)_{F} \cdot \gamma_{F}\left(u, \frac{1}{2} \psi\right)^{-1}, & \text { if } a \in F^{\times} .\end{cases}
$$

We next calculate $\lambda(1, U(a))$. Since $g=(1, U(a))$ stabilizes $\mathbb{Y}^{\prime}$,

$$
\lambda(g)=\gamma_{F}\left(\frac{1}{2} \psi \circ q\right), \quad q:=q\left(\mathbb{V}^{\Delta}, \mathbb{Y}^{\prime}, \mathbb{V}^{\Delta} g\right) .
$$

Working in the $F$-basis given in (5.5),

$$
\begin{aligned}
\mathbb{V}^{\Delta} & =\left\{\left(0,0,0,0, y_{1}, y_{2}, y_{3}, y_{4}\right)\right\}, \\
\mathbb{Y}^{\prime} & =\left\{\left(0,0, y_{1}, y_{2}, y_{3}, y_{4}, 0,0\right)\right\}, \\
\mathbb{V}^{\triangle} g & =\left\{\left(0,0,-a y_{3}, \frac{a}{u} y_{4}, y_{1}+\frac{a}{2} y_{3}, y_{2}-\frac{a u}{2} y_{4}, y_{3}, y_{4}\right)\right\} .
\end{aligned}
$$

If $a=0$, then $\lambda(1, U(0))=1$, and the lemma holds. It remains to prove the assertion for the case where $a \in F^{\times}$. Then we have that the sum of the pairwise intersections of $\mathbb{V}^{\Delta}, \mathbb{Y}^{\square}, \mathbb{V}^{\Delta} g$ is $\mathbb{R}=\{(0,0,0,0, *, *, 0,0)\}$. The perpendicular 
subspace to $\mathbb{R}$ in $\mathbb{V} \square$ is equal to $\mathbb{R}^{\perp}=\{(0,0, *, *, *, *, *, *)\}$ so that

$$
\begin{aligned}
\left(\mathbb{V}^{\Delta}\right)_{\mathbb{R}} & =\left\{\left(0,0,0,0,0,0, y_{1}, y_{2}\right)\right\}, \\
\left(\mathbb{Y}^{\prime}\right)_{\mathbb{R}} & =\left\{\left(0,0, y_{1}, y_{2}, 0,0,0,0\right)\right\}, \\
\left(\mathbb{V}^{\triangle} g\right)_{\mathbb{R}} & =\left\{\left(0,0,-a y_{1}, \frac{a}{u} y_{2}, 0,0, y_{1}, y_{2}\right)\right\},
\end{aligned}
$$

where the subscript $\mathbb{R}$ denotes the corresponding image in $\mathbb{R}^{\perp} / \mathbb{R}$. It is clear from the above equations that

$$
\begin{aligned}
& \left(\mathbb{Y}^{\prime}\right)_{\mathbb{R}}\left(\begin{array}{cc}
1 & b \\
0 & 1
\end{array}\right)=\left(\mathbb{V}^{\Delta} g\right)_{\mathbb{R}}, \\
& \quad \text { where }\left(\begin{array}{ll}
1 & b \\
0 & 1
\end{array}\right) \in P_{\left(\mathbb{V}^{\Delta}\right)_{\mathbb{R}}} \subset \operatorname{Sp}\left(\mathbb{R}^{\perp} / \mathbb{R}\right), \text { for } b=\left(\begin{array}{cc}
-\frac{1}{a} & 0 \\
0 & \frac{u}{a}
\end{array}\right) .
\end{aligned}
$$

By definition, $q=\left(\mathbb{Y}^{\prime}\right)_{\mathbb{R}}$ with the symmetric bilinear form given by

$$
q\left(\left(x_{1}, x_{2}\right),\left(y_{1}, y_{2}\right)\right)=-\frac{1}{a} x_{1} y_{1}+\frac{u}{a} x_{2} y_{2} .
$$

Therefore we have

$$
\operatorname{dim} q=2, \quad \operatorname{det} q=-\frac{u}{a^{2}}, \quad h_{F}(q)=\left(-\frac{1}{a}, \frac{u}{a}\right)_{F} .
$$

Observe that $\left(-\frac{1}{a}, \frac{u}{a}\right)_{F}=(-a, a u)_{F}(-a, a)_{F}=(-a, u)_{F}$, and so

$$
\begin{aligned}
\lambda(1, U(a)) & =\gamma_{F}\left(\frac{1}{2} \psi\right)^{2} \cdot \gamma_{F}\left(-\frac{u}{a^{2}}, \frac{1}{2} \psi\right) \cdot\left(-\frac{1}{a}, \frac{u}{a}\right)_{F} \\
& =\gamma_{F}\left(-1, \frac{1}{2} \psi\right)^{-1} \cdot \gamma_{F}\left(-u, \frac{1}{2} \psi\right) \cdot(-a, u)_{F} .
\end{aligned}
$$

Finally, we have

$\mathbf{s}(1, U(a))=(u, a)_{F} \cdot \gamma_{F}\left(u, \frac{1}{2} \psi\right)^{-1} \cdot \gamma_{F}\left(-1, \frac{1}{2} \psi\right)^{-1} \cdot \gamma_{F}\left(-u, \frac{1}{2} \psi\right) \cdot(-a, u)_{F}=1$.

LEMMA 5.19. We have

$$
\mathbf{s}(1, W)=(u,-1)_{F} \cdot \gamma_{F}\left(u, \frac{1}{2} \psi\right) .
$$

In particular, if $\operatorname{ord}(u)$ is even, then

$$
\mathbf{s}(1, W)=1 .
$$


Proof. The matrix $W$ sends $w_{1} \mapsto-w_{1}$ and $w_{2} \mapsto-w_{2}$. Recalling that $i(W)$ acts linearly on $W_{0}$ and trivially on $W_{0}^{-}$, it is a straightforward computation to see that

$$
\begin{aligned}
i(1, W) & =\left(\begin{array}{cccc}
\frac{1}{2} & -\frac{1}{2} & \frac{1}{4} & -\frac{1}{4} \\
\frac{1}{2} & \frac{1}{2} & \frac{1}{4} & \frac{1}{4} \\
-1 & 1 & \frac{1}{2} & -\frac{1}{2} \\
-1 & -1 & \frac{1}{2} & \frac{1}{2}
\end{array}\right) \\
& =\left(\begin{array}{cccc}
1 & -1 & -\frac{1}{4} & \frac{1}{4} \\
1 & 1 & -\frac{1}{4} & -\frac{1}{4} \\
0 & 0 & \frac{1}{2} & -\frac{1}{2} \\
0 & 0 & \frac{1}{2} & \frac{1}{2}
\end{array}\right)\left(\begin{array}{cccc} 
& & 1 & \\
& & & 1 \\
-1 & & & \\
& -1 &
\end{array}\right)\left(\begin{array}{cccc}
2 & 0 & -1 & 0 \\
0 & 2 & 0 & -1 \\
0 & 0 & \frac{1}{2} & 0 \\
0 & 0 & 0 & \frac{1}{2}
\end{array}\right) .
\end{aligned}
$$

Therefore we have $x(i(1, W))=\frac{1}{8}$ and $j(i(1, W))=2$, and by Definition 5.4,

$$
\hat{s}(1, W)=\xi\left(\frac{1}{8}\right) \cdot\left((u,-1)_{F} \cdot \gamma_{F}\left(u, \frac{1}{2} \psi\right)\right)^{-2}=(u,-2)_{F} .
$$

We next calculate $\lambda(1, W)$. With respect to the symplectic basis given in (5.5), the image of $i(1, W)$ in $\operatorname{Sp}\left(\mathbb{V}^{\square}\right)$ is

$$
g=\left(\begin{array}{cccccccc}
\frac{1}{2} & & -\frac{1}{2} & & \frac{1}{4} & & -\frac{1}{4} & \\
& \frac{1}{2} & & \frac{1}{2} & & -\frac{u}{4} & & \frac{u}{4} \\
\frac{1}{2} & & \frac{1}{2} & & \frac{1}{4} & & \frac{1}{4} & \\
& \frac{1}{2} & & \frac{1}{2} & & -\frac{u}{4} & & -\frac{u}{4} \\
-1 & & 1 & & \frac{1}{2} & & -\frac{1}{2} & \\
& u & & -u & \frac{1}{2} & & -\frac{1}{2} \\
-1 & & -1 & & \frac{1}{2} & & \frac{1}{2} & \\
& u & & u & & \frac{1}{2} & & \frac{1}{2}
\end{array}\right) \in \operatorname{Sp}\left(\mathbb{V}^{\square}\right) .
$$

By definition,

$$
\lambda(g)=\gamma_{F}\left(\frac{1}{2} \psi \circ q\left(\mathbb{V}^{\Delta}, \mathbb{Y}^{\prime \square} g^{-1}, \mathbb{Y}^{\prime \square}\right)\right) \cdot \gamma_{F}\left(\frac{1}{2} \psi \circ q\left(\mathbb{V}^{\Delta}, \mathbb{Y}^{\prime \square}, \mathbb{V}^{\Delta} g\right)\right) .
$$

In the following, when calculating $q\left(\mathbb{Y}_{1}, \mathbb{Y}_{2}, \mathbb{Y}_{3}\right)$, we write $\mathbb{R}$ to denote the sum of the pairwise intersections of the $\mathbb{Y}_{i}$ and write $\left(\mathbb{Y}_{i}\right)_{\mathbb{R}}$ to mean the image of $\mathbb{Y}_{i}$ in $\mathbb{R}^{\perp} / \mathbb{R}$. We have

$$
\begin{aligned}
\mathbb{V}^{\Delta} & =\left\{\left(0,0,0,0, y_{1}, y_{2}, y_{3}, y_{4}\right)\right\}, \\
\mathbb{Y}^{\prime} g^{-1} & =\left\{\left(y_{1}, y_{2}, y_{3}, y_{4}, \frac{1}{2} y_{3},-\frac{1}{2 u} y_{4}, \frac{1}{2} y_{1},-\frac{1}{2 u} y_{2}\right)\right\}, \\
\mathbb{Y}^{\prime} \square & =\left\{\left(0,0, y_{1}, y_{2}, y_{3}, y_{4}, 0,0\right)\right\},
\end{aligned}
$$


which implies that $\mathbb{R}=\{(0,0, *, *, *, *, 0,0)\}$ and hence

$$
\gamma_{F}\left(\frac{1}{2} \psi \circ q\left(\mathbb{V}^{\Delta}, \mathbb{Y}^{\prime \square} g^{-1}, \mathbb{Y}^{\prime \square}\right)\right)=1 \text {. }
$$

Now we calculate the second factor of $\lambda(1, W)$. We have

$$
\begin{aligned}
\mathbb{V}^{\Delta} & =\left\{\left(0,0,0,0, y_{1}, y_{2}, y_{3}, y_{4}\right)\right\}, \\
\mathbb{Y}^{\prime} & =\left\{\left(0,0, y_{1}, y_{2}, y_{3}, y_{4}, 0,0\right)\right\}, \\
\mathbb{V}^{\Delta} g & =\left\{\left(y_{1}, y_{2}, y_{3}, y_{4},-\frac{1}{2} y_{1}, \frac{1}{2 u} y_{2},-\frac{1}{2} y_{3}, \frac{1}{2 u} y_{4}\right)\right\},
\end{aligned}
$$

and hence $\mathbb{R}=\{(0,0,0,0, *, *, 0,0)\}, \mathbb{R}^{\perp}=\{(0,0, *, *, *, *, *, *)\}$. This implies that

$$
\begin{aligned}
\left(\mathbb{V}^{\triangle}\right)_{\mathbb{R}} & =\left\{\left(0,0,0,0,0,0, y_{1}, y_{2}\right)\right\}, \\
\left(\mathbb{Y}^{\prime}\right)_{\mathbb{R}} & =\left\{\left(0,0, y_{1}, y_{2}, 0,0,0,0\right)\right\}, \\
\left(\mathbb{V}^{\triangle} g\right)_{\mathbb{R}} & =\left\{\left(0,0, y_{1}, y_{2}, 0,0,-\frac{1}{2} y_{1}, \frac{1}{2 u} y_{2}\right)\right\},
\end{aligned}
$$

and we have

$$
\left(\mathbb{Y}^{\prime}\right)_{\mathbb{R}}\left(\begin{array}{ll}
1 & b \\
0 & 1
\end{array}\right)=\left(\mathbb{V}^{\Delta} g\right)_{\mathbb{R}}, \quad \text { for } b=\left(\begin{array}{cc}
-\frac{1}{2} & \\
& \frac{1}{2 u}
\end{array}\right) .
$$

It follows that

$$
\begin{aligned}
\gamma_{F}\left(\frac{1}{2} \psi \circ q\left(\mathbb{V}^{\Delta}, \mathbb{Y}^{\prime \square}, \mathbb{V}^{\Delta} g\right)\right) & =\gamma_{F}\left(\frac{1}{2} \psi\right)^{2} \cdot \gamma_{F}\left(-\frac{1}{4 u}, \frac{1}{2} \psi\right) \cdot\left(-\frac{1}{2}, \frac{1}{2 u}\right)_{F} \\
& =\gamma_{F}\left(u, \frac{1}{2} \psi\right) \cdot(2, u)_{F} .
\end{aligned}
$$

Putting together Equations (5.6)-(5.8), we have

$$
\begin{aligned}
\mathbf{s}(1, W) & =\hat{s}(1, W) \cdot \lambda(1, W)=(u,-2)_{F} \cdot \gamma_{F}\left(u, \frac{1}{2} \psi\right) \cdot(u, 2)_{F} \\
& =(u,-1)_{F} \cdot \gamma_{F}\left(u, \frac{1}{2} \psi\right) .
\end{aligned}
$$

To see the final assertion, first observe that if $\operatorname{ord}(u)$ is even, then either $E$ is split or unramified over $F$. In either case, $(u,-1)_{F}=1$. By [R93, Proposition A.11], $\operatorname{ord}(u)$ even implies that $\gamma_{F}\left(u, \frac{1}{2} \psi\right)=1$. 
Lemma 5.20. Let $a \in F$. Then

$$
\mathbf{s}(1, D(-1)) \mathbf{s}(1, W) \mathbf{s}(1, U(a)) \mathbf{s}(1, W)=1 .
$$

Proof. We have $\mathbf{s}(1, D(-1)) \mathbf{s}(1, W) \mathbf{s}(1, U(a)) \mathbf{s}(1, W)=(u,-1)_{F}\left((u,-1)_{F} \gamma_{F}\right.$ $\left.\left(u, \frac{1}{2} \psi\right)\right)^{2}=1$.

LEMMA 5.21. If $F=\mathbb{R}$ and $E=\mathbb{C}$, then for any $(\alpha, g) \in R$,

$$
\mathbf{s}(\alpha, g)=\xi\left(\alpha^{-1}\right) .
$$

Proof. Since $(\alpha, D(1, \mathrm{Nm}(\alpha)))$ stabilizes $\mathbb{Y}^{\prime}$,

$$
\mathbf{s}(\alpha, g)=\mathbf{s}(\alpha, D(1, \mathrm{Nm}(\alpha))) \cdot \mathbf{s}\left(1, D\left(1, \mathrm{Nm}(\alpha)^{-1}\right) g\right) .
$$

By Lemma 5.17, to prove the desired assertion, it remains to show that $\mathbf{s}(1, g)=1$ for $g \in \mathrm{SL}_{2}(\mathbb{R})$. But this follows from [R93, Proposition A.10(1)].

5.6.2. A splitting $\mathbf{s}^{\prime}$ of $z_{\mathbb{Y}^{\prime}}$. As in the previous subsection, write $D(a, d):=$ $\operatorname{diag}(a, d)$.

LEMMA 5.22. (i) If $(D(a, d), \alpha) \in \mathrm{G}\left(\mathrm{GL}_{2}(F) \times E^{\times}\right)$, then

$$
\mathbf{s}^{\prime}(D(a, d), \alpha)=\xi^{\prime}\left(-\left(a^{-1} \alpha-1\right)\left(d^{-1} \alpha-1\right)\right) .
$$

In particular, we have $\mathbf{s}^{\prime}\left(D\left(a, a^{-1}\right), 1\right)=(u, a)_{F}$ and $\mathbf{s}^{\prime}(D(1, \operatorname{Nm}(\alpha)), \alpha)$ $=\xi^{\prime}(\alpha)$.

(ii) For $a \in F$, we have $\mathbf{s}^{\prime}(1, U(a))=1$.

(iii) We have $\mathbf{s}^{\prime}(1, W)=(u,-1)_{F} \cdot \gamma_{F}\left(u, \frac{1}{2} \psi\right)$. In particular, if $\operatorname{ord}(u) \in 2 \mathbb{Z}$, then $\mathbf{s}^{\prime}(1, W)=1$.

(iv) For $a \in F$, we have $\mathbf{s}^{\prime}(1, D(-1)) \mathbf{s}^{\prime}(1, W) \mathbf{s}^{\prime}(1, U(a)) \mathbf{s}^{\prime}(1, W)=1$.

(v) If $F=\mathbb{R}$ and $E=\mathbb{C}$, then $\mathbf{s}^{\prime}(\alpha, g)=\xi^{\prime}(\alpha)$.

Proof. The proof of (i) is similar to Lemma 5.17 except that $(D(a, d), \alpha)$ sends $w_{1} \mapsto a^{-1} \alpha w_{1}$ and $w_{2} \mapsto d^{-1} \alpha w_{2}$. Thus the image of $(D(a, d), \alpha)$ in $\mathrm{U}_{E}(\operatorname{Res} V+$ $\operatorname{Res} V^{-}$) with respect to the basis $\mathbf{w}_{1}, \mathbf{w}_{2}, \mathbf{w}_{1}^{*}, \mathbf{w}_{2}^{*}$ is 


$$
\left(\begin{array}{cccc}
\frac{a^{-1} \alpha+1}{2} & 0 & 0 & \frac{a^{-1} \alpha-1}{4} \\
0 & \frac{d^{-1} \alpha+1}{2} & -\frac{d^{-1} \alpha-1}{4} & 0 \\
0 & -\left(d^{-1} \alpha-1\right) & \frac{d^{-1} \alpha+1}{2} & 0 \\
-\left(a^{-1} \alpha-1\right) & 0 & 0 & \frac{a^{-1} \alpha+1}{2}
\end{array}\right)
$$

To be more precise, this proof is the proof of Lemma 5.17 except with $a$ replaced by $a^{-1}, b$ replaced by $b^{-1}$, and $\alpha^{-1}$ replaced by $\alpha$. The proofs of the remaining parts are exactly the same as that of the analogous statements for $\mathbf{s}$.

\section{Global theta lifts}

In this section, we examine the global theta lifts in the similitude seesaw (4.4) in comparison to automorphic induction. Let $\chi$ be a Hecke character and recall that its automorphic induction $\pi_{\chi}$ to $\mathrm{GL}_{2}\left(\mathbb{A}_{F}\right)$ has a Jacquet-Langlands transfer to $B^{\times}$if and only if the following condition holds:

If $B_{v}$ is ramified, then $\chi_{v}$ does not factor through $\mathrm{Nm}: E_{v}^{\times} \rightarrow F_{v}^{\times}$.

We write $\pi_{\chi}^{B}$ to denote the Jacquet-Langlands transfer to $B^{\times}$if the pair $(B, \chi)$ satisfies the above condition, and we set $\pi_{\chi}^{B}=0$ otherwise. The main theorem of this section is as follows.

THEOREM 6.1. The theta lifts $\Theta(\chi \cdot \xi)$ from $\mathrm{GU}_{B}(V)$ to $\mathrm{GU}_{B}\left(W^{*}\right) \cong B^{\times}$ and $\Theta^{\prime}\left(\overline{\chi^{\prime} \cdot \xi^{\prime-1}}\right)$ from $\mathrm{GU}_{E}(W)$ to $\mathrm{GU}_{E}(\operatorname{Res} V) \cong\left(E^{\times} \times\left(B^{\prime}\right)^{\times}\right) / F^{\times}$can be described in terms of automorphic induction and the Jacquet-Langlands correspondence:

$$
\Theta(\chi \cdot \xi) \cong \pi_{\chi}^{B} \quad \text { and } \quad \Theta^{\prime}\left(\overline{\chi^{\prime} \cdot \xi^{\prime-1}}\right)^{\vee} \cong \pi_{\chi^{\prime}}^{B^{\prime}} \otimes\left(\chi^{\prime-1} \cdot \xi^{\prime}\right),
$$

where the right-hand side is viewed as a representation of $\mathrm{GU}_{E}(\operatorname{Res} V)$ descended from $\left(B_{\mathbb{A}}^{\prime}\right)^{\times} \times \mathbb{A}_{E}^{\times}$.

To prove Theorem 6.1, we will need two arguments.

(1) If $\Theta(\chi \cdot \xi)=0$, then $\pi_{\chi}^{B}=0$.

(2) If $\Theta(\chi \cdot \xi) \neq 0$, then $\Theta(\chi \cdot \xi) \cong \pi_{\chi}^{B}$. 
To prove (1), we will need to make use of the theory of doubling zeta integrals. Since the nonvanishing of the global theta lift $\Theta(\chi \cdot \xi)$ is determined by the nonvanishing of local doubling zeta integrals (Section 6.2), the crux of (1) is to establish that the local zeta integral is vanishing only if the local theta lift is. To prove (2), we will need to calculate the local theta lift from $\mathrm{GU}(1)_{v}$ to $\mathrm{GU}(2)_{v}$ at all places where $\mathrm{GU}(2)_{v} \cong \mathrm{GU}(1,1)_{v}$. After showing that $\Theta(\chi \cdot \xi)$ must be cuspidal if it is nonzero, we apply Jacquet-Langlands [JL] to conclude.

6.1. Theta lifts with similitudes. We first recall some general properties of Weil representations. Denote by $\omega_{\psi}$ and $\omega_{\psi}^{\square}$ the Weil representations of $\operatorname{Mp}(\mathbb{V})$ on $\mathcal{S}(\mathbb{X})$ and of $\operatorname{Mp}\left(\mathbb{V}^{\square}\right)$ on $\mathcal{S}\left(\mathbb{X}^{\square}\right)=\mathcal{S}(\mathbb{X}) \otimes \mathcal{S}(\mathbb{X})$. We have a natural map

$$
\tilde{\imath}: \operatorname{Mp}(\mathbb{V}) \times \operatorname{Mp}(\mathbb{V}) \rightarrow \operatorname{Mp}\left(\mathbb{V}^{\square}\right)
$$

inducing $\left(z_{1}, z_{2}\right) \mapsto z_{1} \bar{z}_{2}$ on $\mathbb{C}^{1}$, and $\omega_{\psi}, \omega_{\psi}^{\square}$ enjoy the following compatibility:

$$
\omega_{\psi}^{\square} \circ \tilde{\imath} \cong \omega_{\psi} \otimes\left(\omega_{\psi} \circ \tilde{\mathfrak{j}}_{\mathbb{Y}}\right),
$$

where $\tilde{\mathfrak{j}}_{\mathbb{Y}}$ is the automorphism of $\operatorname{Mp}(\mathbb{V})_{\mathbb{Y}}=\operatorname{Sp}(\mathbb{V}) \times \mathbb{C}^{1}$ defined by

$$
\tilde{\mathfrak{j}}_{\mathbb{Y}}(g, z)=\left(\mathfrak{j}_{\mathbb{Y}}(g), z^{-1}\right), \quad \mathfrak{j}_{\mathbb{Y}}(g)=d_{\mathbb{Y}}(-1) \cdot g \cdot d_{\mathbb{Y}}(-1) .
$$

We make the following definitions:

$$
\begin{array}{ll}
G^{\square}:=\mathrm{GU}_{B}\left(V^{\square}\right) & G^{\square \prime}:=\mathrm{GU}_{E}\left(W^{\square}\right) \\
G:=\mathrm{GU}_{B}(V)^{\circ} \cong E^{\times} \cong \mathrm{GU}_{E}\left(V_{0}\right) & G^{\prime}:=\mathrm{GU}_{E}(W) \\
H:=\mathrm{GU}_{B}\left(W^{*}\right) \cong B^{\times} \subset \mathrm{GU}_{E}\left(W_{0}\right) & H^{\prime}:=\mathrm{GU}_{E}(\operatorname{Res} V) \cong\left(\left(B^{\prime}\right)^{\times} \times E^{\times}\right) / F^{\times} .
\end{array}
$$

Recall that these groups fit into the following seesaws:
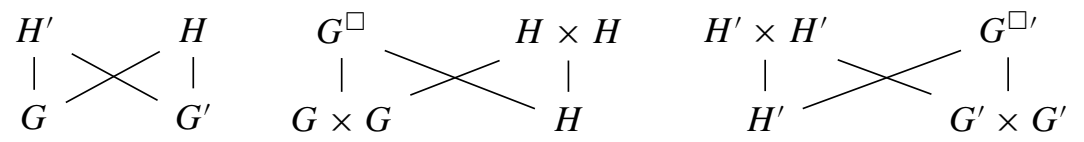

Adding a subscript 1 to any of the above groups indicates that we take the kernel of the similitude character. If $G^{(1)}, \ldots, G^{(n)}$ is a collection of unitary similitude groups, we define

$$
\mathcal{G}_{G^{(1)} \times \cdots \times G^{(n)}}:=\left\{\left(g_{1}, \ldots, g_{n}\right) \in G^{(1)} \times \cdots \times G^{(n)}: v\left(g_{1}\right)=\cdots=v\left(g_{n}\right)\right\} .
$$

We also define $Z:=F^{\times}$and $\mathcal{C}:=\left(\mathbb{A}^{\times}\right)^{2}\left(F^{\times}\right)^{+} \backslash\left(\mathbb{A}^{\times}\right)^{+}$, where

$$
\begin{aligned}
& \left(\mathbb{A}^{\times}\right)^{+}:=v(G(\mathbb{A})) \cap v(H(\mathbb{A}))=v\left(G^{\prime}(\mathbb{A})\right) \cap v\left(H^{\prime}(\mathbb{A})\right)=\operatorname{Nm}_{E / F}\left(\mathbb{A}_{E}^{\times}\right), \\
& \left(F^{\times}\right)^{+}:=F^{\times} \cap\left(\mathbb{A}^{\times}\right)^{+} .
\end{aligned}
$$


Adding a superscript + to any of the groups $G, H, G^{\prime}, H^{\prime}$ means we take the preimage of $\left(\mathbb{A}^{\times}\right)^{+}$(or $\left(F^{\times}\right)^{+}$and so on) under the similitude map.

Fix sections

$$
\mathcal{C} \rightarrow G(\mathbb{A})^{+}, \quad \mathcal{C} \rightarrow H(\mathbb{A})^{+}, \quad \mathcal{C} \rightarrow G^{\prime}(\mathbb{A})^{+}, \quad \mathcal{C} \rightarrow H^{\prime}(\mathbb{A})^{+},
$$

of the natural surjections induced by the similitude character. We write $g_{c}, h_{c}$, $g_{c}^{\prime}, h_{c}^{\prime}$ for the images of $c \in \mathcal{C}$ under these sections. The following lemma is straightforward.

LEMMA 6.2. The similitude character induces isomorphisms

$$
\begin{array}{ll}
Z(\mathbb{A}) G_{1}(\mathbb{A}) G(F)^{+} \backslash G(\mathbb{A})^{+} \cong \mathcal{C}, & Z(\mathbb{A}) H_{1}(\mathbb{A}) H(F)^{+} \backslash H(\mathbb{A})^{+} \cong \mathcal{C}, \\
Z(\mathbb{A}) G_{1}^{\prime}(\mathbb{A}) G^{\prime}(F)^{+} \backslash G^{\prime}(\mathbb{A})^{+} \cong \mathcal{C}, & Z(\mathbb{A}) H_{1}^{\prime}(\mathbb{A}) H^{\prime}(F)^{+} \backslash H^{\prime}(\mathbb{A})^{+} \cong \mathcal{C}
\end{array}
$$

and

$$
\begin{aligned}
H(\mathbb{A}) /\left(H(F) H(\mathbb{A})^{+}\right) & \cong H^{\prime}(\mathbb{A}) /\left(H^{\prime}(F) H^{\prime}(\mathbb{A})^{+}\right) \cong \operatorname{Gal}(E / F), \\
G^{\square}(\mathbb{A}) /\left(G^{\square}(F) G^{\square}(\mathbb{A})^{+}\right) & \cong G^{\square \prime}(\mathbb{A}) /\left(G^{\square \prime}(F) G^{\square \prime}(\mathbb{A})^{+}\right) \cong \operatorname{Gal}(E / F) .
\end{aligned}
$$

Recall that in Section 5 (see Definitions 5.4 and 5.8), for each place $v$ of $F$, we defined splittings of $z_{\mathbb{Y}_{v}}$ and $z_{\mathbb{Y}_{v}^{\square}}$ on certain unitary groups. Recall also that the discussion in Section 5.5 allowed us to multiply the local splittings to obtain global splittings of $z_{\mathbb{Y}}$

$$
s: \mathcal{G}_{G \times H}(\mathbb{A}) \rightarrow \mathbb{C}^{1}, \quad s^{\prime}: \mathcal{G}_{H^{\prime} \times G^{\prime}}(\mathbb{A}) \rightarrow \mathbb{C}^{1},
$$

and global splittings of $z_{\mathbb{Y}} \square$

$$
s^{\square}: \mathcal{G}_{G} \square_{\times H}(\mathbb{A}) \rightarrow \mathbb{C}^{1}, \quad s^{\square \prime}: \mathcal{G}_{H^{\prime} \times G^{\square}}(\mathbb{A}) \rightarrow \mathbb{C}^{1} .
$$

These allow us to define corresponding Weil representations $\omega_{\psi}, \omega_{\psi}^{\prime}, \omega_{\psi}^{\square}, \omega_{\psi}^{\square}$. By Proposition 5.16,

$$
\begin{gathered}
\omega_{\psi}^{\square}\left(g_{1}, g_{2}, h\right)=\omega_{\psi}\left(g_{1}, h\right) \otimes \xi\left(\operatorname{det}\left(g_{2}, h\right)\right) \overline{\omega_{\psi}\left(g_{2}, h\right)}, \quad\left(g_{1}, g_{2}, h\right) \in \mathcal{G}_{G \times G \times H}(\mathbb{A}), \\
\omega_{\psi}^{\square \prime}\left(h, g_{1}, g_{2}\right)=\omega_{\psi}^{\prime}\left(h, g_{1}\right) \otimes \xi^{\prime}\left(\operatorname{det}\left(h, g_{2}\right)\right) \overline{\omega_{\psi}^{\prime}\left(h, g_{2}\right)}, \quad\left(h, g_{1}, g_{2}\right) \in \mathcal{G}_{H^{\prime} \times G^{\prime} \times G^{\prime}}(\mathbb{A}), \\
\omega_{\psi}\left(g, g^{\prime}\right)=\xi(g) \xi^{\prime}\left(g^{\prime}\right) \omega_{\psi}^{\prime}\left(g, g^{\prime}\right), \quad\left(g, g^{\prime}\right) \in \mathcal{G}_{G \times G^{\prime}}(\mathbb{A}) .
\end{gathered}
$$

Define a theta distribution

$$
\Theta: \mathcal{S}(\mathbb{X}(\mathbb{A})) \rightarrow \mathbb{C}, \quad \varphi \mapsto \sum_{x \in \mathbb{X}(F)} \varphi(x) .
$$


Let $\varphi \in \mathcal{S}(\mathbb{X}(\mathbb{A}))$ and let $\chi$ be a Hecke character. For $h=h_{1} h_{c} \in H(\mathbb{A})^{+}$where $h_{1} \in H_{1}(\mathbb{A})$, define

$$
\theta_{\varphi}(\chi)(h):=\int_{G_{1}(F) \backslash G_{1}(\mathbb{A})} \Theta\left(\omega_{\psi}\left(g_{1} g_{c}, h\right) \varphi\right) \chi\left(g_{1} g_{c}\right) d g_{1} .
$$

Here, $d g=\prod_{v} d g_{1, v}$ is the Tamagawa measure on $G_{1}(\mathbb{A})$. Note that $\theta_{\varphi}(\chi)(\gamma h)=$ $\theta_{\varphi}(\chi)(h)$ for $\gamma \in H(F) \cap H(\mathbb{A})^{+}$and $h \in H(\mathbb{A})^{+}$. By declaring

$$
\theta_{\varphi}(\chi)(\gamma h)=\theta_{\varphi}(\chi)(h), \quad \text { for all } \gamma \in H(F) \text { and } h \in H(\mathbb{A})^{+},
$$

we obtain an automorphic form on the subgroup $H(F) H(\mathbb{A})^{+}$of $H(\mathbb{A})$. Let $\varphi \in$ $\mathcal{S}(\mathbb{X}(\mathbb{A}))$ and let $\chi^{\prime}$ be a Hecke character. For $h^{\prime}=h_{1}^{\prime} h_{c}^{\prime} \in H^{\prime}(\mathbb{A})^{+}$where $h_{1}^{\prime} \in$ $H_{1}^{\prime}(\mathbb{A})$, define

$$
\theta_{\varphi}^{\prime}\left(\chi^{\prime}\right)\left(h^{\prime}\right):=\int_{G_{1}^{\prime}(F) \backslash G_{1}^{\prime}(\mathbb{A})} \overline{\Theta\left(\omega_{\psi}^{\prime}\left(h^{\prime}, g_{1}^{\prime} g_{c}^{\prime}\right) \varphi\right)} \chi^{\prime}\left(g_{1}^{\prime} g_{c}^{\prime}\right) d g_{1}^{\prime} .
$$

Here, $d g_{1}^{\prime}=\prod_{v} d g_{1, v}^{\prime}$ is the Tamagawa measure on $G_{1}^{\prime}(\mathbb{A})$.

Let $\Theta_{+}(\chi)$ be the automorphic representation of $H(F) H(\mathbb{A})^{+}$generated by $\theta_{\varphi}(\chi)$ for $\varphi \in \mathcal{S}(\mathbb{X}(\mathbb{A}))$ and let $\Theta_{+}^{\prime}\left(\chi^{\prime}\right)$ be the automorphic representation of $H^{\prime}(F) H^{\prime}(\mathbb{A})^{+}$generated by $\theta_{\varphi}^{\prime}\left(\chi^{\prime}\right)$ for all $\varphi \in \mathcal{S}(\mathbb{X}(\mathbb{A}))$. Define

$$
\left.\Theta(\chi):=\operatorname{Ind}_{H(F) H(\mathbb{A})^{+}}^{H(\mathbb{A})}\left(\Theta_{+}(\chi)\right), \quad \Theta^{\prime}\left(\chi^{\prime}\right):=\operatorname{Ind}_{H^{\prime}(F) H^{\prime}(\mathbb{A})^{+}}^{H^{\prime}\left(\Theta_{+}^{\prime}\right.}\left(\chi^{\prime}\right)\right) .
$$

By Lemma 6.2, $\left[H(\mathbb{A}): H(F) H(\mathbb{A})^{+}\right]=2$, so $\theta_{\varphi}(\chi)$ extends to an automorphic form in $\Theta(\chi)$ via

$$
\theta_{\varphi}(\chi)(h):= \begin{cases}\theta_{\varphi}(\chi)\left(h_{+}\right) & \text {if } h=\gamma h_{+} \text {for } \gamma \in H(F) \text { and } h_{+} \in H(\mathbb{A})^{+}, \\ 0 & \text { otherwise. }\end{cases}
$$

Similarly, $\theta_{\varphi}^{\prime}\left(\chi^{\prime}\right)$ extends to an automorphic form in $\Theta^{\prime}\left(\chi^{\prime}\right)$ by setting

$$
\theta_{\varphi}^{\prime}\left(\chi^{\prime}\right)\left(h^{\prime}\right):= \begin{cases}\theta_{\varphi}^{\prime}\left(\chi^{\prime}\right)\left(h_{+}^{\prime}\right) & \text { if } h^{\prime}=\gamma h_{+}^{\prime} \text { for } \gamma \in H^{\prime}(F) \text { and } h_{+}^{\prime} \in H^{\prime}(\mathbb{A})^{+}, \\ 0 & \text { otherwise. }\end{cases}
$$

The theta lifts for $\omega_{\psi}^{\square}$ and $\omega_{\psi}^{\square \prime}$ are defined analogously.

6.2. The Rallis inner product formula. In this section, we will write down an equation relating the Petersson inner product of a theta lift, to a theta lift to a doubled unitary similitude group. To this end, we will use the doubled seesaws in (5.2) and (6.1). 
For automorphic forms $f_{1}, f_{2}$ on $H(\mathbb{A}) \cong B_{\mathbb{A}}^{\times}$and $f_{1}^{\prime}, f_{2}^{\prime}$ on $H^{\prime}(\mathbb{A}) \cong$ $\left(B_{\mathbb{A}}^{\prime} \times \times \mathbb{A}_{E}^{\times}\right) / \mathbb{A}_{F}^{\times}$, define

$$
\left\langle f_{1}, f_{2}\right\rangle_{H}:=\int_{[H]} f_{1}(h) \cdot \overline{f_{2}(h)} d h, \quad\left\langle f_{1}^{\prime}, f_{2}^{\prime}\right\rangle_{H^{\prime}}:=\int_{\left[H^{\prime}\right]} f_{1}^{\prime}\left(h^{\prime}\right) \cdot \overline{f_{2}^{\prime}\left(h^{\prime}\right)} d h,
$$

where $d h=\prod_{v} d h_{v}$ and $d h^{\prime}=\prod_{v} d h_{v}^{\prime}$ are the Tamagawa measures of $H(\mathbb{A})$ and $H^{\prime}(\mathbb{A})$.

Recall from Proposition 5.16 that the splittings $s: \mathcal{G}_{G \times H}(\mathbb{A}) \rightarrow \mathbb{C}^{1}$ and $s^{\square}: \mathcal{G}_{G \square \times H}(\mathbb{A}) \rightarrow \mathbb{C}^{1}$ enjoy the property that for $\left(g_{1}, g_{2}, h\right) \in \mathcal{G}_{G \times G \times H}$,

$$
s^{\square}\left(g_{1}, g_{2}, h\right)=s\left(g_{1}, h\right) \cdot \overline{s\left(g_{2}, h\right)} \cdot \xi\left(\operatorname{det}\left(i\left(g_{2}, h\right)\right)\right) .
$$

This compatibility implies that for any $h_{1} \in H_{1}, g_{1}, g_{1}^{\prime} \in G_{1}$, and $\left(g_{c}, h_{c}\right) \in$ $\mathcal{G}_{G \times H}(\mathbb{A})$,

$$
\begin{aligned}
& \Theta\left(\omega_{\psi}\left(g_{1} g_{c}, h_{1} h_{c}\right) \varphi_{1}\right) \cdot \overline{\Theta\left(\omega_{\psi}\left(g_{1}^{\prime} g_{c}, h_{1} h_{c}\right) \varphi_{2}\right)} \\
& \quad=\Theta\left(\omega_{\psi}^{\square}\left(\left(g_{1} g_{c}, g_{1}^{\prime} g_{c}\right), h_{1} h_{c}\right) \varphi_{1} \otimes \bar{\varphi}_{2}\right) \cdot \xi\left(\operatorname{det}\left(h_{1} h_{c}\right)\right)^{-1} \cdot \xi\left(g_{1}^{\prime} g_{c}\right)^{2} .
\end{aligned}
$$

Hence for $\varphi_{1}, \varphi_{2} \in \mathcal{S}(\mathbb{X}(\mathbb{A}))$ and Hecke characters $\chi_{1}, \chi_{2}$ of $E^{\times}$, by formally switching the integrals at the equality, we have

$$
\begin{aligned}
&\left\langle\theta_{\varphi_{1}}\left(\chi_{1} \cdot \xi\right), \theta_{\varphi_{2}}\left(\chi_{2} \cdot \xi\right)\right\rangle_{H} \\
&= \int_{\mathcal{C}} \int_{\left[H_{1}\right]} \theta_{\varphi_{1}}\left(\chi_{1} \cdot \xi\right)\left(h_{1} h_{c}\right) \cdot \overline{\theta_{\varphi_{2}}\left(\chi_{2} \cdot \xi\right)\left(h_{1} h_{c}\right)} d h_{1} d c \\
&= \int_{\mathcal{C}} \int_{\left[H_{1}\right]} \int_{\left[G_{1}\right]} \int_{\left[G_{1}\right]} \Theta\left(\omega_{\psi}\left(g_{1} g_{c}, h_{1} h_{c}\right) \varphi_{1}\right)\left(\chi_{1} \xi\right)\left(g_{1} g_{c}\right) \\
& \cdot \overline{\Theta\left(\omega_{\psi}\left(g_{1}^{\prime} g_{c}, h_{1} h_{c}\right) \varphi_{2}\right)\left(\chi_{2} \xi\right)\left(g_{1}^{\prime} g_{c}\right)} d g_{1} d g_{1}^{\prime} d h d c \\
&= \int_{\mathcal{C}} \int_{\left[G_{1}\right]} \int_{\left[G_{1}\right]}\left(\chi_{1} \xi\right)\left(g_{c} g_{c}\right) \cdot\left(\bar{\chi}_{2} \xi\right)\left(g_{1}^{\prime} g_{c}\right) \\
& \cdot \int_{\left[H_{1}\right]} \Theta\left(\omega_{\psi}^{\square}\left(\left(g_{1} g_{c}, g_{1}^{\prime} g_{c}\right), h_{1} h_{c}\right)\left(\varphi_{1} \otimes \bar{\varphi}_{2}\right)\right) \cdot \xi\left(\operatorname{det}\left(h_{1} h_{c}\right)\right)^{-1} d h_{1} d g_{1} d g_{1}^{\prime} d c .
\end{aligned}
$$

The inner integral in Equation (6.6) is the theta lift of $\xi(\mathrm{det})^{-1}$ to $\mathrm{GU}_{B}\left(V^{\square}\right)$, but to make actual sense of the above, one must be careful about convergence issues. In the case that $B$ is division, the quotient $B^{\times} \backslash B_{\mathbb{A}}^{\times}$is compact, and therefore the integral in (6.6) is absolutely convergent. Hence the formal manipulation above is completely justified. We can then use the Siegel-Weil formula together with the theory of doubling integrals [PSR87] to obtain a Rallis inner product formula. In the case that $B$ is split (that is, $B \cong M_{2}(F)$ ), (6.6) does not converge absolutely in general, so the last equality does not make sense. In this case, we use the regularized Siegel-Weil formula of [GQT]. 
6.2.1. The Siegel-Weil formula for division quaternion algebras. In this section, we explain how to obtain a Rallis inner product formula in the case that $B$ is division. For $\varphi \in \mathcal{S}(\mathbb{X} \nabla(\mathbb{A}))$, define

$$
E\left(g, \mathcal{F}_{\varphi}\right)=\sum_{\gamma \in P(F) \backslash \mathrm{U}(1,1)} \mathcal{F}_{\varphi}(\gamma g), \quad \text { where } \mathcal{F}_{\varphi}(g):=\left(\omega_{\psi}^{\square}\left(d\left(\nu(g)^{-1}\right) g\right) \varphi\right)(0) .
$$

This is the value of an Eisenstein series at $s=\frac{1}{2}$. In this case, the Siegel-Weil formula states that for $g, g^{\prime} \in \mathrm{GU}(1)$ such that $v(g)=v\left(g^{\prime}\right)$,

$$
E\left(i\left(g, g^{\prime}\right), \mathcal{F}_{\varphi}\right)=\int_{\left[H_{1}\right]} \Theta\left(\omega_{\psi}^{\square}\left(\left(g, g^{\prime}\right), h\right)\left(\varphi_{1} \otimes \bar{\varphi}_{2}\right)\right) \cdot \xi(\operatorname{det}(h))^{-1} d h,
$$

where $i: \mathrm{G}(\mathrm{U}(1) \times \mathrm{U}(1)) \rightarrow \mathrm{U}(1,1)$ and $\varphi \in \mathcal{S}(\mathbb{V} \nabla(\mathbb{A}))$ is the partial Fourier transform of $\varphi_{1} \otimes \bar{\varphi}_{2} \in \mathcal{S}\left(\mathbb{X}^{\square}(\mathbb{A})\right)$. We now see that, continuing from (6.5) and (6.6), we have

$$
\begin{aligned}
& \left\langle\theta_{\varphi_{1}}\left(\chi_{1} \cdot \xi\right), \theta_{\varphi_{2}}\left(\chi_{2} \cdot \xi\right)\right\rangle_{H} \\
& =\int_{\mathcal{C}} \int_{\left[G_{1}\right]} \int_{\left[G_{1}\right]}\left(\chi_{1} \xi\right)\left(g_{1} g_{c}\right) \cdot\left(\bar{\chi}_{2} \xi\right)\left(g_{1}^{\prime} g_{c}\right) \cdot E\left(i\left(g_{1} g_{c}, g_{1}^{\prime} g_{c}\right), \mathcal{F}_{\varphi}\right) d g_{1} d g_{1}^{\prime} d c .
\end{aligned}
$$

We have $\mathcal{F}_{\varphi}\left(i\left(g_{1} g_{c}, g_{1}^{\prime} g_{c}\right)\right)=\mathcal{F}\left(i\left(g_{1}^{\prime-1} g_{1}, 1\right)\right) \bar{\xi}^{2}\left(g_{1}^{\prime}\right)$, and hence unfolding the above integral and making the substitution $g=g_{1} g_{c}, g^{\prime}=g_{1}^{\prime-1} g_{1}$ gives

$$
=\int_{G_{1}(\mathbb{A})} \int_{[G]}\left(\chi_{1} \xi\right)\left(g g^{\prime}\right) \cdot\left(\overline{\chi_{2} \xi}\right)(g) \cdot \mathcal{F}_{\varphi}(i(g, 1)) d g d g^{\prime} .
$$

The Tamagawa measure on $G_{1}(\mathbb{A})$ can be written as a product of local measures $d g_{1, v}$ on $G_{1, v}$ times a global factor $\rho_{F} / \rho_{E}$ (see Section 2.1). Hence if $\chi_{1}=\chi_{2}=\chi$ and $\varphi_{1}=\varphi_{2}=\phi=\otimes_{v} \phi_{v}$, we have

$$
\begin{aligned}
\left\langle\theta_{\varphi}(\chi \cdot \xi), \theta_{\phi}(\chi \cdot \xi)\right\rangle_{H} & =\int_{G_{1}(\mathbb{A})} \mathcal{F}_{\varphi}(i(g, 1))\left\langle(\chi \xi)\left(g^{\prime}\right)(\chi \xi),(\chi \xi)\right\rangle_{[G]} d g^{\prime} \\
& =\frac{\rho_{F}}{\rho_{E}} \cdot \prod_{v} Z\left(\frac{1}{2}, \mathcal{F}_{\varphi_{v}}, \chi_{v}\right)
\end{aligned}
$$

where

$$
Z\left(\frac{1}{2}, \mathcal{F}_{\varphi_{v}}, \chi_{v}\right):=\int_{G_{1, v}}\left\langle\omega_{\psi}\left(g_{1, v}\right) \phi, \phi\right\rangle \cdot\left(\chi_{v} \xi_{v}\right)\left(g_{1, v}\right) d g_{1, v}
$$

6.2.2. The regularized Siegel-Weil formula for $\left(E^{\times}, \mathrm{GL}(2)\right)$. In this section, we follow [GQT] and describe how to make sense of (6.6) and obtain a Rallis inner product formula in the case that $B$ is split. We will need to translate between 
the quaternionic unitary groups $\left(\mathrm{GU}_{B}(V)^{\circ}, \mathrm{GU}_{B}\left(W^{*}\right)\right) \cong\left(E^{\times}, \mathrm{GL}_{2}(F)\right)$ and the dual reductive pair $(\mathrm{GO}(2), \mathrm{GSp}(2)) \cong\left(E^{\times}, \mathrm{GL}_{2}(F)\right)$. In the notation of [GQT], we have $n=m=2, r=1, \epsilon=1$, which puts us in the second term range since $1<2 \leqslant 2 \cdot 1$.

Recall that we have an embedding

$$
\mathrm{G}\left(\mathrm{U}_{B}(V)^{\circ}, \mathrm{U}_{B}\left(W^{*}\right)\right) \hookrightarrow \mathrm{G}\left(\mathrm{U}_{E}\left(V_{0}\right) \times \mathrm{U}_{E}\left(W_{0}\right)\right) .
$$

When $B$ is split, then there is a decomposition $W_{0}=W_{1}+W_{2}$ of the $E$-space $W_{0}$ into isotropic subspaces of dimension 1 . Set

$$
\mathbb{X}^{\prime}=\operatorname{Res}_{E / F}\left(V_{0} \otimes W_{1}\right), \quad \mathbb{Y}^{\prime}=\operatorname{Res}_{E / F}\left(V_{0} \otimes W_{2}\right)
$$

so that $\mathbb{V}=\mathbb{X}^{\prime}+\mathbb{Y}^{\prime}$ forms a complete polarization. In Section 5.6, we explicated a splitting $\mathbf{s}$ of $z_{\mathbb{Y}^{\prime}}$. Comparing $\mathbf{s}$ to the splitting

$$
s_{(\mathrm{O}(2), \mathrm{Sp}(2))}: \mathrm{G}(\mathrm{O}(2) \times \mathrm{Sp}(2))_{\mathbb{A}} \rightarrow \mathbb{C}^{1}
$$

defined in [K94], we see that for $\alpha \in E^{\times}, a \in F^{\times}$, and $a^{\prime} \in F$,

$$
\begin{aligned}
\mathbf{s}(\alpha, d(\mathrm{Nm}(\alpha))) & =\xi(\alpha)^{-1} \cdot s_{(\mathrm{O}(2), \operatorname{Sp}(2))}(\alpha, d(\mathrm{Nm}(\alpha))), \\
\mathbf{s}\left(1, \operatorname{diag}\left(a, a^{-1}\right)\right) & =\xi(a)^{-1} \cdot s_{(\mathrm{O}(2), \operatorname{Sp}(2))}\left(1, \operatorname{diag}\left(a, a^{-1}\right)\right), \\
\mathbf{s}\left(1,\left(\begin{array}{cc}
1 & a^{\prime} \\
0 & 1
\end{array}\right)\right) & =s_{(\mathrm{O}(2), \operatorname{Sp}(2))}\left(1,\left(\begin{array}{cc}
1 & a^{\prime} \\
0 & 1
\end{array}\right)\right), \\
\mathbf{s}\left(1,\left(\begin{array}{cc}
0 & 1 \\
-1 & 0
\end{array}\right)\right) & =s_{(\mathrm{O}(2), \operatorname{Sp}(2))}\left(1,\left(\begin{array}{cc}
0 & 1 \\
-1 & 0
\end{array}\right)\right) .
\end{aligned}
$$

Now set $V_{0}^{\nabla}:=\left\{(v,-v): v \in V_{0}\right\}$ and $V_{0}^{\Delta}:=\left\{(v, v): v \in V_{0}\right\}$ so that

$$
\mathbb{V} \nabla=\operatorname{Res}_{E / F}\left(V_{0}^{\nabla} \otimes W_{0}\right), \quad \mathbb{V}^{\Delta}=\operatorname{Res}_{E / F}\left(V_{0}^{\triangle} \otimes W_{0}\right)
$$

gives a complete polarization $\mathbb{V}^{\square}=\mathbb{V}^{\nabla}+\mathbb{V}^{\Delta}$ of the doubled symplectic space. Let $\hat{s}_{(\mathrm{O}(2,2), \mathrm{Sp}(2))}$ denote the splitting of $z_{\mathbb{V} \Delta}$ defined in [K94] and define

$$
\begin{aligned}
s_{(\mathrm{O}(2,2), \mathrm{Sp}(2))}(h, g):= & \hat{s}_{(\mathrm{O}(2,2), \mathrm{Sp}(2))}(h, g) \cdot \lambda_{\mathbb{Y}^{\prime} \square \rightsquigarrow \mathbb{V} \Delta}^{-1}(g, h) \\
& \text { for }(g, h) \in \mathrm{G}(\mathrm{O}(2,2), \mathrm{Sp}(2)),
\end{aligned}
$$

where $\lambda:=\lambda_{\mathbb{Y}^{\prime}} \square_{\rightsquigarrow \mathbb{V} \Delta}$ is the change-of-polarization function defined in Lemma 5.3. Then using Proposition 5.16(a),

$$
\begin{aligned}
\hat{s}\left(g_{1}, g_{2}, h\right) & =\mathbf{s}^{\square}\left(g_{1}, g_{2}, 1\right) \cdot \lambda\left(g_{1}, g_{2}, h\right) \\
& =\mathbf{s}\left(g_{1}, h\right) \cdot \overline{\mathbf{s}\left(g_{2}, h\right)} \cdot \xi\left(\operatorname{det}\left(i\left(g_{2}, h\right)\right)\right) \cdot \lambda\left(g_{1}, g_{2}, h\right)
\end{aligned}
$$




$$
\begin{aligned}
= & s_{(\mathrm{O}(2), \mathrm{Sp}(2))}\left(g_{1}, h\right) \xi\left(g_{1}\right)^{-1} \cdot \overline{s_{(\mathrm{O}(2), \mathrm{Sp}(2))}\left(g_{2}, h\right) \xi\left(g_{2}\right)^{-1}} \\
& \cdot \xi\left(g_{2}\right)^{-2} \xi(\operatorname{det}(h)) \cdot \lambda\left(g_{1}, g_{2}, h\right) \\
= & s_{(\mathrm{O}(2), \mathrm{Sp}(2))}\left(g_{1}, h\right) \cdot \overline{s_{(\mathrm{O}(2), \mathrm{Sp}(2))}\left(g_{2}, h\right)} \\
& \cdot \xi\left(g_{1}\right)^{-1} \xi\left(g_{2}\right)^{-1} \xi(\operatorname{det}(h)) \cdot \lambda\left(g_{1}, g_{2}, h\right) \\
= & s_{(\mathrm{O}(2,2), \mathrm{Sp}(2))}\left(g_{1}, g_{2}, h\right) \cdot \xi\left(g_{1}\right)^{-1} \xi\left(g_{2}\right)^{-1} \cdot \lambda\left(g_{1}, g_{2}, h\right) \\
= & \hat{s}_{(\mathrm{O}(2,2), \mathrm{Sp}(2))}\left(g_{1}, g_{2}, h\right) \cdot \xi\left(g_{1}\right)^{-1} \xi\left(g_{2}\right)^{-1} .
\end{aligned}
$$

Define $P_{O} \subset \mathrm{GO}\left(\operatorname{Res}_{E / F} V_{0}^{\square}\right) \cong \mathrm{GO}(2,2)$ to be the stabilizer of the totally isotropic subspace $\operatorname{Res}_{E / F} V_{0}^{\Delta}$ of $\operatorname{Res}_{E / F} V_{0}^{\square}$. For $\phi \in \mathcal{S}(\mathbb{V} \nabla(\mathbb{A}))$, define the Siegel-Weil sections

$$
\begin{aligned}
\Phi(\phi)(g) & :=\left(\omega_{\psi}^{\square}(g) \phi\right)(0), \quad \text { for } g \in \mathrm{GO}(2,2)_{\mathbb{A}} \subset \mathrm{GU}_{E}\left(V_{0}^{\square}\right)_{\mathbb{A}}, \\
\Phi^{\mathrm{O}, \mathrm{Sp}}(\phi)(g) & :=\left(\omega_{\psi}^{\mathrm{O}(2,2), \mathrm{Sp}(2)}(g) \phi\right)(0), \quad \text { for } g \in \mathrm{GO}(2,2)_{\mathbb{A}} .
\end{aligned}
$$

Observe that $\Phi(\phi)(g)=\hat{s}(g) \cdot \hat{s}_{(\mathrm{O}(2,2), \mathrm{Sp}(2))}(g)^{-1} \cdot \Phi^{\mathrm{O}, \mathrm{Sp}}(\phi)(g)$. We make the analogous definitions for the local objects $\Phi_{v}\left(\phi_{v}\right)$ and $\Phi_{v}^{\mathrm{O}, \mathrm{Sp}}\left(\phi_{v}\right)$. The SiegelWeil section $\Phi^{\mathrm{O}, \mathrm{Sp}}(\phi) \in \operatorname{Ind}_{P_{O}}^{\mathrm{GO}(2,2)}(\mathrm{det}) \cdot|\mathrm{det}|^{1 / 2}$ determines a standard section $\Phi_{S}^{\mathrm{O}, \mathrm{Sp}}(\phi) \in \operatorname{Ind}_{P_{O}}^{\mathrm{GO}(2,2)}(\mathrm{det}) \cdot|\operatorname{det}|^{s}$ and we may form the associated Eisenstein series

$$
E\left(s, \Phi^{\mathrm{O}, \mathrm{Sp}}(\phi)\right)(g):=\sum_{\gamma \in P_{O}(F) \backslash \mathrm{GO}(2,2)} \Phi_{s}^{\mathrm{O}, \mathrm{Sp}}(\gamma g), \quad \text { for } g \in \mathrm{GO}(2,2)_{\mathbb{A}} .
$$

Define

$$
Z(s, \Phi, \chi):=\int_{[\mathrm{G}(\mathrm{O}(2) \times \mathrm{O}(2))]} E(s, \Phi)\left(g_{1}, g_{2}\right) \cdot \chi\left(g_{1}\right) \cdot \bar{\chi}\left(g_{2}\right) d g_{1} d g_{2} .
$$

If $\Phi=\otimes_{v} \Phi_{v}$, define

$$
Z_{v}\left(s, \Phi_{v}, \chi_{v}\right)=\int_{E_{v}^{1}} \Phi_{v}\left(g_{v}, 1\right) \cdot \chi_{v}\left(g_{v}\right) d g_{v}
$$

By construction of the Tamagawa measure of $\mathbb{A}_{E}^{1}$ (see Section 2.1), one has

$$
Z(s, \Phi, \chi):=\frac{\rho_{F}}{\rho_{E}} \cdot \prod_{v} Z_{v}\left(s, \Phi_{v}, \chi_{v}\right)
$$

Define the partial Fourier transform $\delta: \mathcal{S}\left(\mathbb{X}^{\prime}(\mathbb{A})\right) \rightarrow \mathcal{S}(\mathbb{V} \nabla(\mathbb{A}))$ by

$$
\delta(\varphi)(u)=\int_{\left(\left(\mathbb{V} \Delta \cap \mathbb{Y}^{\prime} \square\right) \backslash \mathbb{V} \Delta\right)(\mathbb{A})} \varphi(x) \psi\left(\frac{1}{2}(\langle\langle x, y\rangle\rangle-\langle\langle u, v\rangle\rangle)\right) d v,
$$

where we write $u+v=x+y$ with $u \in \mathbb{V} \nabla(\mathbb{A}), v \in \mathbb{V}^{\triangle}(\mathbb{A}), x \in \mathbb{N}^{\prime}(\mathbb{A})$, $y \in \mathbb{Y}^{\prime}(\mathbb{A})$, and $d v$ is the Tamagawa measure. 
Observe that if $\phi \in \mathcal{S}(\mathbb{V} \nabla(\mathbb{A}))$ is the partial Fourier transform of $\varphi_{1} \otimes \bar{\varphi}_{2}$ for $\varphi_{1}, \varphi_{2} \in \mathcal{S}\left(\mathbb{X}^{\prime}(\mathbb{A})\right)$, then for the Siegel-Weil section $\Phi=\Phi^{\mathrm{O}, \mathrm{Sp}}\left(\delta\left(\varphi_{1} \otimes \bar{\varphi}_{2}\right)\right)$, we have

$$
\begin{aligned}
Z_{v}\left(\frac{1}{2}, \Phi_{v}, \chi_{v}\right) & =\operatorname{vol}\left(E_{v}^{1}\right) \int_{E_{v}^{1}} \Phi^{\mathrm{O}, \mathrm{Sp}}\left(\delta\left(\varphi_{1} \otimes \bar{\varphi}_{2}\right)\right)\left(i\left(g_{1, v}, 1\right)\right) \cdot \chi_{v}\left(g_{v}\right) d g_{v} \\
& =\operatorname{vol}\left(E_{v}^{1}\right) \int_{E_{v}^{1}}\left(\omega_{\psi}^{\mathrm{O}(2,2), \mathrm{Sp}(2)}\left(g_{v}, 1\right) \delta\left(\varphi_{1} \otimes \bar{\varphi}_{2}\right)\right)(0) \cdot \chi_{v}\left(g_{v}\right) d g_{v} \\
& =\operatorname{vol}\left(E_{v}^{1}\right) \int_{E_{v}^{1}}\left(\omega_{\psi}^{\square}\left(g_{v}, 1\right) \delta\left(\varphi_{1} \otimes \bar{\varphi}_{2}\right)\right)(0) \cdot \chi_{v}\left(g_{v}\right) \cdot \xi_{v}\left(g_{v}\right) d g_{v} \\
& =\operatorname{vol}\left(E_{v}^{1}\right) \int_{E_{v}^{1}}\left\langle\omega_{\psi}\left(g_{v}\right) \varphi_{1}, \varphi_{2}\right\rangle \cdot\left(\chi_{v} \xi_{v}\right)\left(g_{v}\right) d g_{v} .
\end{aligned}
$$

Proposition 6.3. For $\varphi_{1}, \varphi_{2} \in \mathcal{S}\left(\mathbb{X}^{\prime}(\mathbb{A})\right)$, we have

$$
\left\langle\theta_{\varphi_{1}}(\chi \xi), \theta_{\varphi_{2}}(\chi \xi)\right\rangle=\frac{\rho_{F}}{\rho_{E}} \cdot \prod_{v} Z_{v}\left(\frac{1}{2}, \Phi_{v}^{\mathrm{O}, \mathrm{Sp}}\left(\delta\left(\varphi_{1} \otimes \bar{\varphi}_{2}\right)\right), \chi_{v}\right) .
$$

Proof. We use (6.8) to translate between our setting and that of [GQT, Proposition 11.1]. We have

$$
\begin{aligned}
&\left\langle\theta_{\varphi_{1}}(\chi \cdot \xi), \theta_{\varphi_{2}}(\chi \cdot \xi)\right\rangle_{H} \\
&=\int_{\mathcal{C}} \int_{\left[H_{1}\right]} \theta_{\varphi_{1}}(\chi \cdot \xi)\left(h_{1} h_{c}\right) \cdot \overline{\theta_{\varphi_{2}}(\chi \cdot \xi)\left(h_{1} h_{c}\right)} d h_{1} d c \\
&=\int_{\mathcal{C}} \int_{\left[H_{1}\right]} \int_{\left[G_{1}\right]} \int_{\left[G_{1}\right]} \Theta\left(\omega_{\psi}\left(g_{1} g_{c}, h_{1} h_{c}\right) \varphi_{1}\right)(\chi \xi)\left(g_{1} g_{c}\right) \\
& \cdot \overline{\Theta\left(\omega_{\psi}\left(g_{1}^{\prime} g_{c}, h_{1} h_{c}\right) \varphi_{2}\right)(\chi \xi)\left(g_{1}^{\prime} g_{c}\right)} d g_{1} d g_{1}^{\prime} d h d c \\
&= \int_{\mathcal{C}} \int_{[\mathrm{Sp}(2)]} \int_{[\mathrm{O}(2)]} \int_{[\mathrm{O}(2)]} \Theta\left(\omega_{\psi}^{\mathrm{O}, \mathrm{Sp}}\left(g_{1} g_{c}, h_{1} h_{c}\right) \varphi_{1}\right)(\chi \xi)\left(g_{1} g_{c}\right) \\
& \cdot \Theta\left(\omega_{\psi}^{\mathrm{O}, \mathrm{Sp}}\left(g_{1}^{\prime} g_{c}, h_{1} h_{c}\right) \varphi_{2}\right)(\chi \xi)\left(g_{1}^{\prime} g_{c}\right) \cdot \xi^{-1}\left(g_{1}\right) \bar{\xi}^{-1}\left(g_{1}^{\prime}\right) d g_{1} d g_{1}^{\prime} d h d c \\
&= \operatorname{Val}_{s=1 / 2} \int_{\mathcal{C}} \int_{[\mathrm{O}(2)]} \int_{[\mathrm{O}(2)]} E\left(s, \Phi_{\mathrm{O}(2,2, \mathrm{Sp}(2))}\left(\delta\left(\varphi_{1} \otimes \bar{\varphi}_{2}\right)\right)\right)\left(g_{1} g_{c}, g_{1}^{\prime} g_{c}\right) \\
& \cdot \chi\left(g_{1} g_{c}\right) \cdot \bar{\chi}\left(g_{1}^{\prime} g_{c}\right) d g_{1} d g_{1}^{\prime} d c \\
&= \operatorname{Val}_{s=1 / 2} Z\left(s, \Phi\left(\delta\left(\varphi_{1} \otimes \bar{\varphi}_{2}\right)\right), \chi\right) .
\end{aligned}
$$

6.3. Local doubling zeta integrals. Let $v$ be a nonsplit place of $F$. For notational convenience, we drop all subscripts $v$ in this section. We preemptively 
note that the notation we use to describe the zeta integrals in this section differs from the notation used to describe the same (local) zeta integrals in the rest of the paper. We learned the proof of Proposition 6.6 from Ichino. Similar arguments appear in [GI14].

Consider the Siegel parabolic subgroup

$$
P=\left\{\left(\begin{array}{cc}
a & * \\
0 & \left(a^{*}\right)^{-1}
\end{array}\right) \in \mathrm{GL}_{2}(E)\right\} \subset \mathrm{U}(1,1),
$$

and for any unitary character $\eta: \mathrm{U}(1) \rightarrow \mathbb{C}^{1}$, consider the functional

$$
Z\left(s, \eta, \xi^{2}\right): I\left(s, \xi^{2}\right) \rightarrow \mathbb{C}, \quad \mathcal{F} \mapsto \int_{E^{1}} \mathcal{F}(i(g, 1)) \eta(g) d g
$$

where $\iota: \mathrm{U}(1) \times \mathrm{U}(1) \rightarrow \mathrm{U}(1,1)$ is the natural map and

$$
\begin{aligned}
I\left(s, \xi^{2}\right) & :=\operatorname{Ind}_{P}^{\mathrm{U}(1,1)}\left(\xi^{2} \cdot|\cdot|^{s}\right) \\
& :=\left\{\begin{array}{l|c}
\mathcal{F}: \mathrm{U}(1,1) \rightarrow \mathbb{C}(p g)=\xi^{2}(a)|a|_{E}^{s+1 / 2} \mathcal{F}(g) \\
\text { for all } g \in \mathrm{U}(1,1) \text { and } p=\left(\begin{array}{cc}
a & * \\
0 & \bar{a}^{-1}
\end{array}\right) \in P
\end{array}\right\}
\end{aligned}
$$

is the normalized principal series representation. One has an intertwining operator

$$
M\left(s, \xi^{2}\right): I\left(s, \xi^{2}\right) \rightarrow I\left(-s, \bar{\xi}^{-2}\right) \cong I\left(-s, \xi^{2}\right)
$$

given by

$$
M\left(s, \xi^{2}\right) \mathcal{F}(g)=\int_{N_{P}} \mathcal{F}(w n g) d n,
$$

where $w=\operatorname{diag}(1,-1)$ and $N_{P}$ is the unipotent radical of the parabolic $P$.

Following Lapid-Rallis (see also Gan-Ichino, Section 10), after normalizing the intertwining operator by some rational function $c_{\psi}\left(s, \xi^{2}\right)$,

$$
M_{\psi}^{\mathrm{LR}}\left(s, \xi^{2}\right):=c_{\psi}\left(s, \xi^{2}\right) M(s, \xi)
$$

has a functional equation of the shape

$$
Z\left(-s, \eta, \xi^{2}\right)\left(M_{\psi}^{\mathrm{LR}}\left(s, \xi^{2}\right) \mathcal{F}\right)=* \cdot \gamma\left(s+\frac{1}{2}, \eta, \bar{\xi}, \psi\right) \cdot Z\left(s, \eta, \xi^{2}\right)(\mathcal{F}),
$$

where $*$ denotes some nonzero factors. In particular, if we understand the behavior of the intertwining operator $M(s, \eta)$ and if $\gamma\left(s_{0}+\frac{1}{2}, \eta\right) \neq 0$, the functional equation gives a relation between the nonvanishing of $Z\left(-s_{0}, \eta, \xi^{2}\right)$ and the nonvanishing of $Z\left(s_{0}, \eta, \xi^{2}\right)$. 
We take a short detour to examine when the local theta lift to the nonsplit unitary group U(2) vanishes. Define

$$
V_{n}^{+}:=\mathbb{H}_{n}, \quad V_{n}^{-}:=D \oplus \mathbb{H}_{n-1},
$$

where $\mathbb{H}_{n}$ is the $2 n$-dimensional split Hermitian $E$-space and $D$ is the nonsplit quaternion algebra over $F$ viewed as a 2-dimensional Hermitian $E$-space via $\langle x, y\rangle=\operatorname{pr}_{E}\left(x^{*} y\right)$. For a character $\eta: \mathrm{U}(1) \cong E^{1} \rightarrow \mathbb{C}^{1}$, denote its theta lift to $\mathrm{U}\left(V_{n}^{ \pm}\right)$by $\Theta_{V_{n}^{ \pm}}(\eta)$. To make this tower 'compatible' one takes the Weil representation for $\mathrm{U}(1) \times \mathrm{U}\left(V_{n}^{+}\right)$to be such that the splitting on $\mathrm{U}(1)$ is given by $\xi$. In particular, the Weil representation on $\mathrm{U}(1) \times \mathrm{U}\left(V_{0}^{+}\right)=\mathrm{U}(1) \times\{1\}$ is given by the 1-dimensional representation $\xi$. The first occurrence of the theta lift in the towers $\left\{\mathrm{U}\left(V_{n}^{+}\right): n \geqslant 0\right\},\left\{\mathrm{U}\left(V_{n}^{-}\right): n \geqslant 0\right\}$ is defined to be

$$
n^{+}=\min \left\{n: \Theta_{V_{n}^{+}}(\eta) \neq 0\right\}, \quad n^{-}=\min \left\{n: \Theta_{V_{n}^{-}}(\eta) \neq 0\right\} .
$$

The following result is a special case of a theorem of Sun-Zhu [SZ15].

Theorem $6.4($ Sun-Zhu $) . n^{+}(\eta)+n^{-}(\eta)=2$.

We can describe the first occurrence in this setting more explicitly. By the compatible choice of splittings in the tower of unitary groups $\mathrm{U}\left(V_{n}^{+}\right)$, we have that $\Theta_{V_{0}^{+}}(\chi \xi) \neq 0$ if and only if $\chi$ is the trivial character. Hence we must necessarily be in the setting $n^{+}(\chi \xi)+n^{-}(\chi \xi)=0+2$, and in particular, $\Theta_{V_{1}^{-}}(\chi \xi)=0$.

Now suppose that $\chi$ is nontrivial. Then by the previous paragraph, $\Theta_{V_{0}^{+}}(\chi \xi)$ $=0$. We now argue that $\Theta_{V_{1}^{+}}(\chi \xi) \neq 0$. One explicit way to see this is as follows. Let $V_{1}^{+}=V_{1}^{\nabla}+V_{1}^{\Delta}$ be a decomposition of $V_{1}^{+}$into totally isotropic $E$-subspaces. For the Schwartz function $\varphi(x)=\chi(x) \mathbb{1}_{\mathcal{O}_{E}^{\times}}(x) \in \mathcal{S}\left(\operatorname{Res}_{E / F} V_{1}^{\nabla}\right)$, we have

$$
\int_{E^{1}}\left(\omega_{\psi}(g) \varphi\right)(0) \cdot(\chi \xi)(g) d g \neq 0,
$$

which proves that there is a nontrivial $E^{1}$-equivariant map

$$
\left(\mathcal{S}\left(\operatorname{Res}_{E / F} V_{1}^{\nabla}\right), \omega_{\psi}\right) \rightarrow(\mathbb{C}, \chi \xi) .
$$

Hence $\Theta_{V_{1}^{+}}(\chi \xi) \neq 0$ by the definition of the local theta lift. This now implies that we must necessarily be in the setting $n^{+}(\chi \xi)+n^{-}(\chi \xi)=1+1$, and $\Theta_{V_{1}^{-}}(\chi \xi) \neq 0$.

In summary, the above arguments prove the following lemma.

LEMMA 6.5. (a) $\Theta_{V_{1}^{-}}(\chi \xi) \neq 0$ if and only if $\chi: E^{1} \rightarrow \mathbb{C}^{\times}$is nontrivial.

(b) If $\chi: E^{1} \rightarrow \mathbb{C}^{1}$ is nontrivial, $\Theta_{V_{1}^{+}}(\chi \xi) \neq 0$. 
We now discuss the relationship between the theory of the doubling zeta integral and the local theta correspondence. Consider the two doubling seesaws for $V_{1}^{+}$and $V_{1}^{-}$:

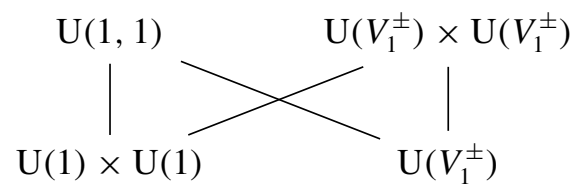

If we have $\mathrm{U}(1,1)=\mathrm{U}(W)$, then one has a decomposition $W=W_{1}+W_{2}$ of $W$ into 1-dimensional isotropic $E$-spaces, and hence by viewing $V_{1}^{ \pm}$as the $F$-space $\operatorname{Res}_{E / F}\left(W_{1} \otimes_{E} V_{1}^{ \pm}\right)=\operatorname{Res}_{E / F}\left(V_{1}^{ \pm}\right)$, the Weil representation $\omega_{\psi}^{\square}$ for $\mathrm{U}(1,1) \times$ $\mathrm{U}\left(V_{1}^{ \pm}\right)$can then be modeled on the space of Schwartz functions $\mathcal{S}\left(V_{1}^{ \pm}\right)$. Define

$$
\mathcal{S}\left(V_{1}^{ \pm}\right) \rightarrow I\left(\frac{1}{2}, \xi^{2}\right), \quad \varphi \mapsto\left(g \mapsto\left(\omega_{\psi}^{\square}(i(g, 1)) \varphi\right)(0)\right),
$$

where $i: \mathrm{U}(1) \times \mathrm{U}(1) \rightarrow \mathrm{U}(1,1)$ is the natural map. Let $R\left(V_{1}^{ \pm}\right)$denote the image of this map. Since $\left.\xi^{2}\right|_{F^{\times}}=1$, there is a unique 1-dimensional representation $\widetilde{\xi}^{2}$ of $\mathrm{U}(1,1)$ extending the representation defined by $\left(\begin{array}{cc}a & * \\ 0 & \bar{a}^{-1}\end{array}\right) \mapsto \xi^{2}(a)$. For the 0 -dimensional Hermitian space $V_{0}^{+}$, we define a map

$$
\mathcal{S}\left(V_{0}^{+}\right)=\mathbb{C} \rightarrow I\left(-\frac{1}{2}, \xi^{2}\right), \quad z \mapsto\left(g \mapsto \widetilde{\xi}^{2}(g)\right) .
$$

Let $R\left(V_{0}^{+}\right)$denote the image of this map. We say that $\Theta_{V_{0}^{+}}(\chi \xi) \neq 0$ if and only if $\operatorname{Hom}_{\mathrm{U}(1)}\left(\widetilde{\xi}^{2}, \chi \xi\right) \neq 0$. Since $\widetilde{\xi}^{2}$ is 1-dimensional, we have $\left.\operatorname{Hom}_{\mathrm{U}(1)} \widetilde{\xi}^{2}, \chi \xi\right) \neq 0$ if and only if $\left.Z\left(-\frac{1}{2}, \chi \xi, \xi^{2}\right)\right|_{R\left(V_{0}^{+}\right)} \neq 0$. Observe also that $\Theta_{V_{0}^{+}}(\chi \xi) \neq 0$ if and only if $\chi=1$.

The goal of the remainder of this section is to prove the following.

Proposition 6.6. Let $\xi: \mathbb{A}_{E}^{\times} \rightarrow \mathbb{C}^{1}$ be such that $\left.\xi\right|_{\mathbb{A}_{F}^{\times}}=\epsilon_{E / F}$. Then

$$
\Theta_{V_{1}^{-}}(\chi \xi) \neq\left. 0 \quad \Longrightarrow \quad Z\left(\frac{1}{2}, \chi \xi, \xi^{2}\right)\right|_{R\left(V_{1}^{-}\right)} \neq 0 \text {. }
$$

We first remark that the converse of Proposition 6.6 is true and straightforward to see: If $\left.Z\left(\frac{1}{2}, \chi \xi, \xi^{2}\right)\right|_{R\left(V_{1}^{-}\right)} \neq 0$, then this immediately implies that $\operatorname{Hom}_{\mathrm{U}(1)}\left(\left.\omega_{\psi}^{\square}\right|_{i(\mathrm{U}(1) \times\{1\})},(\chi \xi)^{-1}\right) \neq 0$. But since $\omega_{\psi}^{\square} \cong \omega_{\psi} \otimes \bar{\omega}_{\psi} \xi^{2}$ (see Lemma 5.7) as a representation of $\mathrm{U}(1) \times \mathrm{U}(1)$, we have $\operatorname{Hom}_{\mathrm{U}(1)}\left(\omega_{\psi},(\chi \xi)^{-1}\right) \neq 0$, and so $\Theta_{V_{1}^{-}}(\chi \xi) \neq 0$ by definition.

Before we prove Proposition 6.6, we recall a special case of a theorem of KudlaSweet.

Theorem 6.7 (Kudla-Sweet, [KS97, Theorem 1.2(1),(4)]). (i) $R\left(V_{0}^{+}\right)$is the unique irreducible submodule of $I\left(-\frac{1}{2}, \xi^{2}\right)$. 
(ii) $I\left(-\frac{1}{2}, \xi^{2}\right) / R\left(0, \xi^{2}\right)$ is an irreducible representation of $\mathrm{U}(1,1)$.

(iii) $R\left(V_{1}^{+}\right)=I\left(\frac{1}{2}, \xi^{2}\right)$.

(iv) $R\left(V_{1}^{-}\right)$is the unique maximal submodule of $I\left(\frac{1}{2}, \xi^{2}\right)$ and is irreducible of codimension 1.

We are now ready to prove the proposition.

Proof of Proposition 6.6. By Lemma 6.5(a), we may assume that $\chi_{v}: E_{v}^{1} \rightarrow \mathbb{C}^{\times}$ is nontrivial. Since $\chi \xi \bar{\xi}=\chi$ and $\overline{\chi \xi} \xi=\bar{\chi}$, by the 'Ten Commandments' for $\gamma$-factors [LR05, Theorem 4], we have

$$
L^{S}(s, \chi)=\prod_{v \in S} \gamma_{v}\left(s,(\chi \xi)_{v}, \bar{\xi}_{v}, \psi_{v}\right) \cdot L^{S}(1-s, \bar{\chi}),
$$

where $S$ is a finite set of places containing all the Archimedean places and all places where $\chi_{v}$ is ramified. Now, since $\chi$ is nontrivial, we must have $L^{S}(0, \chi) \neq$ 0 and $L^{S}(1, \bar{\chi}) \neq 0$, and therefore

$$
\gamma_{v}\left(0,(\chi \xi)_{v}, \xi_{v}, \psi_{v}\right) \neq 0
$$

This implies that Equation (6.10) gives

$$
Z\left(\frac{1}{2}, \chi \xi, \xi^{2}\right)\left(M_{\psi}^{\mathrm{LR}}\left(-\frac{1}{2}, \xi^{2}\right)(\mathcal{F})\right)=* \cdot Z\left(-\frac{1}{2}, \chi \xi, \xi^{2}\right)(\mathcal{F}),
$$

where $*$ is nonzero. We now investigate the intertwining operator

$$
M_{\psi}^{\mathrm{LR}}\left(-\frac{1}{2}, \xi\right): I\left(-\frac{1}{2}, \xi^{2}\right) \rightarrow I\left(\frac{1}{2}, \xi^{2}\right)
$$

We refer to Theorem 6.7 for the decomposition of the $\mathrm{U}(1,1)$-representations $I\left( \pm \frac{1}{2}, \xi^{2}\right)$. By [KS97, Proposition 6.4]

$$
\operatorname{ker}\left(M_{\psi}^{\mathrm{LR}}\left(-\frac{1}{2}, \xi^{2}\right)\right)=R\left(0, \xi^{2}\right), \quad \operatorname{Im}\left(M_{\psi}^{\mathrm{LR}}\left(-\frac{1}{2}, \xi^{2}\right)\right)=R\left(V_{1}^{-}\right) .
$$

Since $\chi$ is nontrivial, $\Theta_{V_{0}^{+}}(\chi \xi)=0$, and therefore $\left.Z\left(-\frac{1}{2}, \chi \xi, \xi^{2}\right)\right|_{R\left(V_{0}^{+}\right)}=0$. On the other hand, $Z\left(-\frac{1}{2}, \chi \xi, \xi^{2}\right)$ is a nonzero functional, and therefore one can find $\mathcal{F} \in I\left(-\frac{1}{2}, \xi^{2}\right)$ such that $M_{\psi}^{\mathrm{LR}}\left(-\frac{1}{2}, \xi^{2}\right)(\mathcal{F}) \neq 0$. By Theorem 6.7(iv), it follows that $\left.Z\left(\frac{1}{2}, \xi \chi, \xi^{2}\right)\right|_{R\left(V_{1}^{-}\right)} \neq 0$. 
6.4. Unramified local theta lifts from $\mathbf{G U}(1)$ to $\mathbf{G U}(1,1)$. For convenience of notation, in this subsection, we drop the subscript $v$. We denote by $\bar{x}$ the image of $x \in E$ under the nontrivial involution of $E / F$.

Consider the 2-dimensional $E$-space $V^{\prime}=V_{1}^{\prime}+V_{2}^{\prime}$ with skew-Hermitian form

$$
\left\langle\left(x_{1}, x_{2}\right),\left(y_{1}, y_{2}\right)\right\rangle=\bar{x}_{1} y_{2}+\bar{x}_{2} y_{1}
$$

for $\left(x_{1}, x_{2}\right),\left(y_{1}, y_{2}\right) \in V_{1}^{\prime}+V_{2}^{\prime}$. Then

$$
\begin{aligned}
\mathrm{GU}\left(V^{\prime}\right) & =\mathrm{GU}(1,1) \\
& =\left\{g \in \mathrm{GL}_{2}(E): \bar{g}^{\mathrm{t}}\left(\begin{array}{cc}
0 & 1 \\
-1 & 0
\end{array}\right) g=v(g)\left(\begin{array}{cc}
0 & 1 \\
-1 & 0
\end{array}\right) \text { for some } v(g) \in F^{\times}\right\} .
\end{aligned}
$$

The upper-triangular matrices in $\mathrm{GU}\left(V^{\prime}\right)$ form a parabolic subgroup

$$
P:=\left\{\left(\begin{array}{cc}
a & v^{\prime} a \\
0 & v a
\end{array}\right) \in \mathrm{GL}_{2}(E): a \in E^{\times}, v \in F^{\times}, v^{\prime} \in F\right\} .
$$

Let $P_{F}$ denote the Borel subgroup of $\mathrm{GL}_{2}(F)$ consisting of upper-triangular matrices in $\mathrm{GL}_{2}(F)$. Observe that there are natural inclusions $\mathrm{GL}_{2}(F) \hookrightarrow \mathrm{GU}\left(V^{\prime}\right)$ and $E^{\times} \hookrightarrow \mathrm{GU}\left(V^{\prime}\right)$ given by

$$
\begin{aligned}
\mathrm{GL}_{2}(F) & =\left\{\left(\begin{array}{ll}
a & b \\
c & d
\end{array}\right) \in \mathrm{GU}\left(V^{\prime}\right): a, b, c, d \in F\right\}, \\
E^{\times} & =\left\{\left(\begin{array}{ll}
a & \\
& a
\end{array}\right) \in \mathrm{GU}\left(V^{\prime}\right): a \in E^{\times}\right\} .
\end{aligned}
$$

We have $\mathrm{GU}\left(V^{\prime}\right) \cong\left(\mathrm{GL}_{2}(F) \times E^{\times}\right) / F^{\times}$and $P \cong\left(P_{F} \times E^{\times}\right) / F^{\times}$.

Endow $E$ with the Hermitian form $(x, y)=x \bar{y}$ so that $\mathrm{GU}(E)=\mathrm{GU}(1)=E^{\times}$. Note that the similitude character on $\mathrm{GU}(E)$, which we also denote by $v$, is given by the norm map $E^{\times} \rightarrow F^{\times}$. Now consider the group

$$
R:=\left\{(h, g) \in E^{\times} \times \mathrm{GU}\left(V^{\prime}\right): v(g)=v(h)\right\} .
$$

Endow the 4-dimensional $F$-space $\mathbb{V}^{\prime}=\operatorname{Res}_{E / F}\left(V^{\prime}\right)$ with the symplectic form $\langle\langle v, w\rangle\rangle=\frac{1}{2} \operatorname{Tr}_{E / F}(\langle v, w\rangle)$. There is a natural map

$$
\iota: R \rightarrow \operatorname{Sp}(\mathbb{V}), \quad(h, g) \mapsto\left(v \mapsto h^{-1} v g\right) .
$$

The decomposition $V_{1}^{\prime}+V_{2}^{\prime}$ of $V^{\prime}$ into isotropic subspaces induces a polarization of $\mathbb{V}^{\prime}$ given by

$$
\mathbb{V}^{\prime}=\mathbb{X}^{\prime}+\mathbb{Y}^{\prime}, \quad \text { where } \mathbb{X}^{\prime}=\operatorname{Res}_{E / F}\left(V_{1}^{\prime}\right) \text { and } \mathbb{Y}^{\prime}=\operatorname{Res}_{E / F}\left(V_{2}^{\prime}\right) .
$$


Choose a basis $\mathbf{e}_{1}, \mathbf{e}_{2}, \mathbf{e}_{1}^{*}, \mathbf{e}_{2}^{*}$ of $\mathbb{V}^{\prime}$ such that

$$
\mathbb{X}^{\prime}=F \mathbf{e}_{1}+F \mathbf{e}_{2}, \quad \mathbb{Y}^{\prime}=F \mathbf{e}_{1}^{*}+F \mathbf{e}_{2}^{*}, \quad\left\langle\left\langle\mathbf{e}_{i}, \mathbf{e}_{j}^{*}\right\rangle\right\rangle=\delta_{i j} .
$$

Now assume that we have a splitting $\beta: R \rightarrow \mathbb{C}^{1}$ of $z_{\mathbb{Y}^{\prime}}$. Then

$$
R \rightarrow \operatorname{Mp}\left(\mathbb{V}^{\prime}\right)_{\mathbb{Y}^{\prime}}, \quad g \mapsto(\iota(g), \beta(g))
$$

is a group homomorphism and the Weil representation $\omega_{\psi}$ on $\operatorname{Mp}\left(\mathbb{V}^{\prime}\right)_{\mathbb{Y}^{\prime}}$ pulls back to a representation of $R$, which we also denote by $\omega_{\psi}$.

Abusing notation, define

$$
\beta: E^{\times} \rightarrow \mathbb{C}^{1}, \quad h \mapsto \beta(h, d(\nu(h))) .
$$

Observe that this defines a character since for any $h \in E^{\times}, \iota(h, d(v(h)))$ stabilizes $\mathbb{Y}^{\prime}$. Define

$$
L(h) \phi(x):=\omega_{\psi}(h, d(v(h))) \phi(x)=\beta(h)|h|^{-1 / 2} \phi\left(x h^{-1}\right)
$$

for $h \in E^{\times}$and $\phi \in \mathcal{S}\left(\mathbb{X}^{\prime}\right)$. Then for any $(h, g) \in R$,

$$
\begin{aligned}
\omega_{\psi}(h, g) \phi(x) & =L(h) \omega_{\psi}\left(d\left(v(g)^{-1}\right) g\right) \phi(x) \\
& =\beta(h)|h|^{-1 / 2}\left(\omega_{\psi}\left(d\left(v(g)^{-1}\right) g\right) \phi\right)\left(x h^{-1}\right) .
\end{aligned}
$$

Consider the semidirect product $E^{\times} \ltimes \mathrm{U}\left(V^{\prime}\right)$ with multiplication

$$
\begin{aligned}
\left(h_{1}, g_{1}\right) *\left(h_{2}, g_{2}\right)= & \left(h_{1} h_{2}, d\left(v\left(h_{2}\right)\right) g_{1} d\left(v\left(h_{2}\right)^{-1}\right) g_{2}\right), \\
& \text { where } h \in E^{\times} \text {and } g \in \mathrm{U}\left(V^{\prime}\right) .
\end{aligned}
$$

This defines a group multiplication since the map $d$ is multiplicative and $v$ is a group homomorphism to $F^{\times}$, an Abelian group. It is easy to show the following lemma.

LEMMA 6.8. The Weil representation $\omega_{\psi}$ on $R$ extends to a representation of $E^{\times} \ltimes \mathrm{U}\left(V^{\prime}\right)$ defined by

$$
\omega_{\psi}(h, g)=L(h) \omega_{\psi}(g), \quad h \in E^{\times}, g \in \mathrm{U}\left(V^{\prime}\right) .
$$

In particular, the Weil representation on the quotient

$$
\Theta^{(1)}(\text { triv }):=\mathcal{S}\left(\mathbb{X}^{\prime}\right) / \bigcap_{\alpha \in \operatorname{Hom}_{E^{1}}\left(\mathcal{S}\left(\mathbb{X}^{\prime}\right), \text { triv }\right)} \operatorname{ker}(\alpha)
$$

extends to a representation of $\mathrm{GU}\left(V^{\prime}\right)^{+} \cong\left\{d(v): v \in \mathrm{Nm}\left(E^{\times}\right)\right\} \ltimes \mathrm{U}\left(V^{\prime}\right)$ satisfying

$$
\omega_{\psi}(d(v))=L(h),
$$

where $h \in E^{\times}$is any element such that $v(h)=v$. 
Definition 6.9. For any character $\eta_{0}: F^{\times} \rightarrow \mathbb{C}$ and any $\phi \in \mathcal{S}\left(\mathbb{X}^{\prime}\right)$, define

$\mathcal{F}_{\phi, \eta_{0}}: \mathrm{GU}\left(V^{\prime}\right) \rightarrow \mathbb{C}^{\times}, \quad g \mapsto|v(g)|^{-1 / 2} \eta_{0}(v(g))^{-1}\left(\omega_{\psi}\left(d\left(v(g)^{-1}\right) g\right) \phi\right)(0)$.

The following is straightforward.

LEMMA 6.10. For any $p=\left(\begin{array}{ll}a & b \\ 0 & d\end{array}\right) \in \mathrm{GU}\left(V^{\prime}\right)$,

$$
\mathcal{F}_{\phi, \eta_{0}}(p g)=|a|^{1 / 2}|d|^{-1 / 2} \eta_{0}(\bar{a} d)^{-1} \beta(a)^{-1} \mathcal{F}_{\phi, \eta_{0}}(g)
$$

for all $g \in \mathrm{GU}\left(V^{\prime}\right)$ so that

$$
\mathcal{F}_{\phi, \eta_{0}} \in \operatorname{Ind}_{P}^{\mathrm{GU}\left(V^{\prime}\right)}\left(\tilde{\eta}_{0}\right), \quad \text { where } \tilde{\eta}_{0}\left(\begin{array}{ll}
a & b \\
0 & d
\end{array}\right):=\eta_{0}(\bar{a} d)^{-1} \beta(a)^{-1} \text {. }
$$

In particular, $\left.\mathcal{F}_{\phi, \eta_{0}}\right|_{\mathrm{GSp}(2)}$ is an element of the (normalized) principal series representation

$$
\operatorname{Ind}_{B}^{\mathrm{GSp}(2)}\left(\eta_{0}^{-1} \beta^{-1} \otimes \eta_{0}^{-1}\right) .
$$

LEMMA 6.11. We have a nonzero R-equivariant map

$$
\left(\omega_{\psi}, \mathcal{S}\left(\mathbb{X}^{\prime}\right)\right) \rightarrow \operatorname{Ind}_{P}^{\mathrm{GU}\left(V^{\prime}\right)}\left(\widetilde{\eta}_{0}\right) \otimes\left(\eta_{0}(\mathrm{Nm}) \cdot \beta\right), \quad \phi \mapsto \mathcal{F}_{\phi, \eta_{0}} .
$$

The right-hand side is irreducible and we have an isomorphism

$$
\operatorname{Ind}_{P}^{\mathrm{GU}\left(V^{\prime}\right)}\left(\widetilde{\eta}_{0}\right) \cong \operatorname{Ind}_{P_{F}}^{\mathrm{GL}_{2}(F)}\left(\eta_{0}^{-1} \otimes\left(\eta_{0} \cdot \beta\right)^{-1}\right) \otimes\left(\eta_{0}(\mathrm{Nm}) \cdot \beta\right)^{-1},
$$

where the right-hand side is a representation of $\mathrm{GL}_{2}(F) \times E^{\times}$that descends to the quotient $\left(\mathrm{GL}_{2}(F) \times E^{\times}\right) / F^{\times} \cong \mathrm{GU}\left(V^{\prime}\right)$.

Proof. It is clear by definition that the map is nonzero. For $R$-equivariance,

$$
\begin{aligned}
& \mathcal{F}_{\omega_{\psi}\left(h^{\prime}, g^{\prime}\right) \phi, \eta_{0}}(g) \\
& \quad=|v(g)|^{-1 / 2} \eta_{0}(v(g))^{-1}\left(\omega_{\psi}\left(d\left(v(g)^{-1}\right) g\right) L\left(h^{\prime}\right) \omega_{\psi}\left(d\left(v\left(g^{\prime}\right)^{-1}\right) g^{\prime}\right) \phi\right)(0) \\
& \quad=|v(g)|^{-1 / 2} \eta_{0}(v(g))^{-1}\left(L\left(h^{\prime}\right) \omega_{\psi}\left(d\left(v\left(g g^{\prime}\right)^{-1}\right) g g^{\prime}\right) \phi\right)(0) \\
& \quad=|v(g)|^{-1 / 2} \eta_{0}(v(g))^{-1}\left|v\left(h^{\prime}\right)\right|^{-1 / 2} \beta\left(h^{\prime}\right)\left(\omega_{\psi}\left(d\left(v\left(g g^{\prime}\right)^{-1}\right) g g^{\prime}\right) \phi\right)(0) \\
& \quad=\beta\left(h^{\prime}\right) \eta_{0}\left(v\left(h^{\prime}\right)\right)\left|v\left(g g^{\prime}\right)\right|^{-1 / 2} \eta_{0}\left(v\left(g g^{\prime}\right)\right)^{-1}\left(\omega_{\psi}\left(d\left(v\left(g g^{\prime}\right)^{-1}\right) g g^{\prime}\right) \phi\right)(0) \\
& \quad=\beta\left(h^{\prime}\right) \eta_{0}\left(v\left(h^{\prime}\right)\right) \mathcal{F}_{\phi, \eta_{0}}\left(g g^{\prime}\right) .
\end{aligned}
$$

The last assertion in the lemma holds since $P \cong\left(P_{F} \times E^{\times}\right) / F^{\times}$and $\mathrm{GU}\left(V^{\prime}\right) \cong$ $\left(\mathrm{GL}_{2}(F) \times E^{\times}\right) / F^{\times}$. The representation $\operatorname{Ind}_{P_{F}}^{\mathrm{GL}_{2}(F)}\left(\widetilde{\eta}_{0}\right)$ is irreducible since the character $\eta_{0}^{-1} \beta^{-1} \eta_{0}=\beta^{-1}$ is not $|\cdot|$ or $|\cdot|^{-1}$. It follows that $\operatorname{Ind}_{P}^{\mathrm{GU}\left(V^{\prime}\right)}\left(\widetilde{\eta}_{0}\right)$ is irreducible. 
The map defined in Lemma 6.11 factors through

$$
\Theta^{(1)}(\beta):=\mathcal{S}\left(\mathbb{X}^{\prime}\right) / \bigcap_{\alpha \in \operatorname{Hom}_{E^{1}}\left(\mathcal{S}\left(\mathbb{X}^{\prime}\right), \beta\right)} \operatorname{ker} \alpha,
$$

the largest quotient of $\mathcal{S}\left(\mathbb{X}^{\prime}\right)$ such that $E^{1}$ acts by $\beta$. Note that by construction, $\Theta^{(1)}(\beta)$, as a representation of $\mathrm{U}\left(V^{\prime}\right)$, is the local theta lift of $\beta$ to $\mathrm{U}\left(V^{\prime}\right)$.

There are many extensions of $\Theta^{(1)}(\beta)$ to a representation of $E^{\times} \times \mathrm{GU}\left(V^{\prime}\right)^{+}$, but specifying an action of $E^{\times}$determines such an extension. Explicitly, define $\Theta_{\mathrm{ur}, \beta}\left(\beta \cdot \eta_{0}(\mathrm{Nm})\right)$ to be the unique representation of $\mathrm{GU}\left(V^{\prime}\right)^{+}$such that for $g=$ $\left(\begin{array}{ll}1 & 0 \\ 0 & v\end{array}\right) \in \mathrm{GU}\left(V^{\prime}\right)^{+}$,

$$
\Theta_{\mathrm{ur}, \beta}\left(\beta \cdot \eta_{0}(\mathrm{Nm})\right)(g):=\eta_{0}(\mathrm{Nm}(h))^{-1} \cdot \Theta^{(1)}(\beta)(h, g),
$$

where $h \in E^{\times}$is any element such that $v(h)=v(g)=v$.

THEOREM 6.12 (Rallis). The R-equivariant map in Lemma 6.11 factors through $\Theta_{\mathrm{ur}, \beta}\left(\beta \cdot \eta_{0}(\mathrm{Nm})\right)$ and induces an injective map:

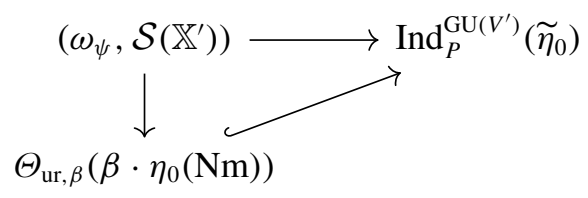

Moreover,

$$
\Theta_{\mathrm{ur}, \beta}\left(\beta \cdot \eta_{0}(\mathrm{Nm})\right) \cong \operatorname{Ind}_{P_{F}}^{\mathrm{GL}_{2}(F)}\left(\eta_{0}^{-1} \epsilon_{E / F} \otimes \eta_{0}^{-1}\right) \otimes\left(\eta_{0}(\mathrm{Nm})^{-1} \cdot \beta^{-1}\right),
$$

where the right-hand side is viewed as a representation of $\mathrm{GL}_{2}(F) \times E^{\times}$that descends to the quotient $\left(\mathrm{GL}_{2}(F) \times E^{\times}\right) / F^{\times} \cong \mathrm{GU}\left(V^{\prime}\right)$.

Proof. This is due to Rallis [R84, Theorem II.1.1]. By the injectivity of

$$
\Theta_{\mathrm{ur}, \beta}\left(\beta \cdot \eta_{0}(\mathrm{Nm})\right) \hookrightarrow \operatorname{Ind}_{P}^{\mathrm{GU}\left(V^{\prime}\right)}\left(\widetilde{\eta}_{0}\right)
$$

and the irreducibility of $\operatorname{Ind}_{P}^{\mathrm{GU}\left(V^{\prime}\right)}\left(\widetilde{\eta}_{0}\right)$, by Lemma 6.11, we have an isomorphism

$$
\Theta_{\mathrm{ur}, \beta}\left(\beta \cdot \eta_{0}(\mathrm{Nm})\right) \cong \operatorname{Ind}_{P_{F}}^{\mathrm{GL}_{2}(F)}\left(\eta_{0}^{-1} \beta^{-1} \otimes \eta_{0}^{-1}\right) \otimes\left(\eta_{0}(\mathrm{Nm})^{-1} \cdot \beta^{-1}\right) .
$$

Finally, by Lemma 5.17, the restriction of $\beta$ to $F^{\times}$is exactly the quadratic character $\epsilon_{E / F}$, and this completes the proof. 
6.5. Proof of Theorem 6.1. In this section, we use the calculations in the preceding sections to prove Theorem 6.1, the main theorem of this section.

Let $\chi$ and $\chi^{\prime}$ be Hecke characters of $E^{\times}$. Recall from Section 6.1 that for every Schwartz function $\varphi \in \mathcal{S}(\mathbb{X}(\mathbb{A}))$, we have automorphic forms $\theta_{\varphi}(\chi)$ and $\theta_{\varphi}^{\prime}\left(\chi^{\prime}\right)$ on the adelic groups $H(\mathbb{A}) \cong B_{\mathbb{A}}^{\times}$and $H^{\prime}(\mathbb{A}) \cong\left(\left(B_{\mathbb{A}}^{\prime}\right)^{\times} \times \mathbb{A}_{E}^{\times}\right) / \mathbb{A}_{F}^{\times}$, respectively. Let $\Theta(\chi)$ denote the automorphic representation of $H(\mathbb{A})$ generated by $\theta_{\varphi}(\chi)$ for all $\varphi \in \mathcal{S}(\mathbb{X}(\mathbb{A}))$ and let $\Theta^{\prime}\left(\chi^{\prime}\right)$ denote the automorphic representation of $H^{\prime}(\mathbb{A})$ generated by $\theta_{\varphi}^{\prime}\left(\chi^{\prime}\right)$ for all $\varphi \in \mathcal{S}(\mathbb{X}(\mathbb{A}))$.

Proposition 6.13. If $\pi_{\chi}^{B} \neq 0$, then $\Theta(\chi \cdot \xi) \neq 0$. Analogously, if $\pi_{\chi^{\prime}}^{B^{\prime}} \neq 0$, then $\Theta^{\prime}\left(\chi^{\prime} \cdot \xi^{\prime}\right) \neq 0$.

Proof. Recall that $\pi_{\chi}^{B} \neq 0$ if and only if $\left.\chi_{v}\right|_{E_{v}^{1}} \neq 1$ for every place $v$ where $B_{v}$ is nonsplit. Let $v$ be such a place, that is, $B_{v}$ is nonsplit and $\left.\chi_{v}\right|_{E_{v}^{1}} \neq 1$. By Lemma 6.5(a), we have $\Theta_{v}\left(\chi_{v} \xi_{v}\right) \neq 0$, and by Proposition 6.6, we have $Z_{v}\left(\frac{1}{2},-, \chi_{v} \xi_{v}\right) \neq 0$. Now let $v$ be a place such that $B_{v}$ is split. By Lemma 6.5(b), we have $\Theta_{v}\left(\chi_{v} \xi_{v}\right) \neq 0$, and by Theorem 6.7(c), we have $Z_{v}\left(\frac{1}{2},-, \chi_{v} \xi_{v}\right) \neq 0$. By the Rallis inner product formula (Proposition 6.3), $\Theta(\chi \cdot \xi) \neq 0$ if and only if all the local zeta integrals $Z_{v}\left(\frac{1}{2},-, \chi_{v} \xi_{v}\right) \neq 0$, and hence we have shown that $\Theta(\chi \cdot \xi) \neq 0$.

LEMMA 6.14. If $\chi, \chi^{\prime}$ are Hecke characters of $\mathbb{A}_{E}^{\times}$whose restriction to $\mathbb{A}_{E}^{1}$ is nontrivial, then $\Theta(\chi \cdot \xi)$ is a cuspidal automorphic representation of $B_{\mathbb{A}}^{\times}$and $\Theta^{\prime}\left(\chi^{\prime} \cdot \xi^{\prime}\right)$ is a cuspidal automorphic representation of $B_{\mathbb{A}}^{\prime} \times$.

Proof. If $B \neq M_{2}(F)$, then the statement holds trivially. Now assume $B=M_{2}(F)$. We would like to prove that for any Schwartz function $\phi \in \mathcal{S}(\mathbb{X}(\mathbb{A}))$,

$$
\int_{F \backslash \mathbb{A}_{F}} \theta_{\phi}(\chi)(\mathbf{n}(b) g) d b=0, \quad \text { where } \mathbf{n}(b):=\left(\begin{array}{ll}
1 & b \\
0 & 1
\end{array}\right) .
$$

Observe that if $g \notin \mathrm{GL}_{2}^{+}\left(\mathbb{A}_{F}\right)$, then $\mathbf{n}(b) g \notin \mathrm{GL}_{2}^{+}\left(\mathbb{A}_{F}\right)$, and hence the integrand in (6.13) is identically zero. Now assume $g \in \mathrm{GL}_{2}^{+}\left(\mathbb{A}_{F}\right)$ and pick $\alpha \in \mathbb{A}_{E}^{\times}$with $\operatorname{Nm}(\alpha)=\operatorname{det}(g)$. Then by definition,

$$
\theta_{\phi}(\chi)(\mathbf{n}(b) g)=\theta_{\omega_{\psi}(\alpha, g) \phi}(\chi)(\mathbf{n}(b)),
$$

and therefore it remains only to show

$$
\int_{F \backslash \mathbb{A}_{F}} \theta_{\phi}(\chi)(\mathbf{n}(b)) d b=0 .
$$


Recall that if $B$ is split, then the 2-dimensional $E$-space $W_{0}$ is a split Hermitian space and one has a decomposition $W_{0}=W_{1}+W_{2}$ into isotropic subspaces of dimension 1 . This induces a complete polarization $\mathbb{V}=\mathbb{X}^{\prime}+\mathbb{Y}^{\prime}$ given by $\mathbb{X}^{\prime}=$ $\operatorname{Res}_{E / F}\left(V_{0} \otimes W_{1}\right)$ and $\mathbb{Y}^{\prime}=\operatorname{Res}_{E / F}\left(V_{0} \otimes W_{2}\right)$. Then $\mathbb{A}_{E}^{1} \subset \mathrm{U}\left(V_{0}\right)$ stabilizes $\mathbb{X}^{\prime}$ and $\mathbb{Y}^{\prime}$, and so for $\alpha \in \mathbb{A}_{E}^{1}, b \in \mathbb{A}_{F}$, and $\phi^{\prime} \in \mathcal{S}\left(\mathbb{X}^{\prime}(\mathbb{A})\right)$,

$$
\omega_{\psi}(\alpha, \mathbf{n}(b)) \phi^{\prime}(x)=\xi^{-1}(\alpha) \cdot \psi\left(\frac{1}{2} b x x^{\mathrm{t}}\right) \cdot \phi^{\prime}(x \alpha) .
$$

We have

$$
\begin{aligned}
\int_{F \backslash \mathbb{A}_{F}} & \theta_{\phi}(\chi)(\mathbf{n}(b)) d b \\
= & \int_{F \backslash \mathbb{A}_{F}} \int_{E^{1} \backslash \mathbb{A}_{E}^{1}} \sum_{x \in \mathbb{X}^{\prime}(F)}\left(\omega_{\psi}(\alpha, \mathbf{n}(b))\right) \phi^{\prime}(x) \cdot(\chi \xi)(\alpha) d \alpha d b \\
= & \int_{E^{1} \backslash \mathbb{A}_{E}^{1}} \sum_{x \in \mathbb{X}^{\prime}(F)} \int_{F \backslash \mathbb{A}_{F}} \xi^{-1}(\alpha) \cdot \psi\left(\frac{1}{2} b x x^{\mathrm{t}}\right) \cdot \phi^{\prime}(x \alpha) \cdot(\chi \xi)(\alpha) d b d \alpha \\
= & \int_{E^{1} \backslash \mathbb{A}_{E}^{1}} \xi^{-1}(\alpha) \cdot \phi^{\prime}(0) \cdot(\chi \xi)(\alpha) d \alpha=\phi^{\prime}(0) \int_{E^{1} \backslash \mathbb{A}_{E}^{1}} \chi(\alpha) d \alpha=0 .
\end{aligned}
$$

THEOREM 6.15. Let $\chi, \chi^{\prime}$ be Hecke characters of $\mathbb{A}_{E}^{\times}$whose restriction to $\mathbb{A}_{E}^{1}$ is nontrivial.

(a) If $\Theta(\chi \cdot \xi)$ is nonzero, then

$$
\Theta(\chi \cdot \xi) \cong \pi_{\chi}^{B}
$$

(b) If $\Theta^{\prime}\left(\overline{\chi^{\prime} \cdot \xi^{-1}}\right)$ is nonzero, then

$$
\Theta^{\prime}\left(\overline{\chi^{\prime} \cdot \xi^{\prime-1}}\right)^{\vee} \cong \pi_{\chi^{\prime}}^{B^{\prime}} \otimes\left(\chi^{\prime} \cdot \xi^{\prime-1}\right),
$$

where the right-hand side is viewed as a representation of $H^{\prime}(\mathbb{A}) \cong$ $\left(\left(B_{\mathbb{A}}^{\prime}\right)^{\times} \times \mathbb{A}_{E}^{\times}\right) / \mathbb{A}_{F}^{\times}$descended from the $\left(B_{\mathbb{A}}^{\prime}\right)^{\times} \times \mathbb{A}_{E}^{\times}$representation written above.

Proof. We prove (a) first. By our normalization (compare the local definition in Section 3.3 to the global definition in Section 6.1), at a place $v$, the local representation corresponding to the global theta lift of $\chi \cdot \xi$ is the local theta lift of $\left(\chi_{v} \cdot \xi_{v}\right)^{-1}$. That is,

$$
\Theta(\chi \cdot \xi)_{v} \cong \Theta_{v}\left(\left(\chi_{v} \cdot \xi_{v}\right)^{-1}\right) \cong \Theta_{v}\left(\chi_{v}^{-1} \cdot \xi_{v}^{-1}\right) .
$$

Theorem 6.12 gives a description of the right-hand side for every place $v$ such that 
- $v$ splits completely in $E$; or

- $v$ lies under a single place $w$ of $E$ and $\chi_{w}: E_{w}^{\times} \rightarrow \mathbb{C}^{\times}$factors through $\mathrm{Nm}: E_{w}^{\times} \rightarrow F_{v}^{\times}$.

For each such place $v$, by Lemma 5.17, we have

$$
\mathbf{s}(\alpha, d(\nu(\alpha)))=\xi(\alpha)^{-1}, \quad \text { for all } \alpha \in E_{v}^{\times} .
$$

Writing $\chi_{v}=\chi_{v, 0}(\mathrm{Nm})$, we have

$$
\Theta_{v}\left(\chi_{v}^{-1} \cdot \xi_{v}^{-1}\right) \cong \Theta_{\mathrm{ur}, \xi_{v}^{-1}}\left(\chi_{v}^{-1} \xi_{v}^{-1}\right) \cong \operatorname{Ind}_{P_{F_{v}}}^{\mathrm{GL}_{2}\left(F_{v}\right)}\left(\chi_{v, 0} \epsilon_{E_{v} / F_{v}} \otimes \chi_{v, 0}\right),
$$

and therefore by Jacquet-Langlands, we have that $\Theta(\chi \cdot \beta) \cong \pi_{\chi}^{B}$.

The proof of $(b)$ is very similar. In this case, because we complex-conjugate the theta kernel in the definition of the global theta lift $\Theta^{\prime}$ (see Section 6.1), we have

$$
\Theta^{\prime}\left(\overline{\chi^{\prime} \cdot \xi^{\prime-1}}\right)_{v}^{\vee} \cong \Theta_{v}\left(\left(\chi_{v}^{\prime} \cdot \xi_{v}^{\prime-1}\right)^{-1}\right)=\Theta_{v}\left(\chi_{v}^{\prime-1} \cdot \xi_{v}^{\prime}\right) .
$$

At every place $v$ of $F$ where everything is unramified, by Lemma 5.22,

$$
\mathbf{s}^{\prime}(d(v(\alpha)), \alpha)=\xi^{\prime}(\alpha), \quad \text { for all } \alpha \in E_{v}^{\times} .
$$

Writing $\chi_{v}^{\prime}=\chi_{v, 0}^{\prime}(\mathrm{Nm})$ at each such place, Theorem 6.12 implies

$$
\Theta_{v}\left(\chi_{v}^{\prime-1} \cdot \xi_{v}^{\prime}\right) \cong \Theta_{\mathrm{ur}, \xi_{v}^{\prime}}\left(\chi_{v}^{\prime-1} \cdot \xi_{v}^{\prime}\right) \cong \operatorname{Ind}_{P_{F_{v}}}^{\mathrm{GL}_{2}\left(F_{v}\right)}\left(\chi_{v, 0}^{\prime} \xi_{v}^{\prime} \otimes \chi_{v, 0}^{\prime}\right) \otimes\left(\chi_{v, 0}^{\prime} \cdot \xi_{v}^{\prime-1}\right) .
$$

Since $\left.\xi_{v}^{\prime}\right|_{F_{v}^{\times}}=\epsilon_{E_{v} / F_{v}}$, therefore by Jacquet-Langlands, $\Theta^{\prime}\left(\overline{\chi^{\prime} \cdot \xi^{\prime-1}}\right)^{\vee} \cong \pi_{\chi^{\prime}}^{B^{\prime}} \otimes$ $\left(\chi^{\prime-1} \cdot \xi^{\prime}\right)$.

Theorem 6.1 now follows from Proposition 6.13 and Theorem 6.15.

Proof of Theorem 6.1. If $\Theta(\chi \cdot \xi)=0$, then by Proposition 6.13, we must have $\pi_{\chi}^{B}=0$ and therefore $\Theta(\chi \cdot \xi)=\pi_{\chi}^{B}$. If $\Theta(\chi \cdot \xi) \neq 0$, then by Theorem 6.15, we must have $\Theta(\chi \cdot \xi) \cong \pi_{\chi}^{B}$. The same argument holds to conclude the desired isomorphism for $\Theta^{\prime}\left(\overline{\chi^{\prime} \cdot \xi^{\prime-1}}\right)$.

6.6. Period identities of $\mathbf{C M}$ forms. We are now ready to prove an identity of toric integrals of automorphic forms in $\pi_{\chi}^{B}$ and $\pi_{\chi^{\prime}}^{B^{\prime}}$. We use the seesaw

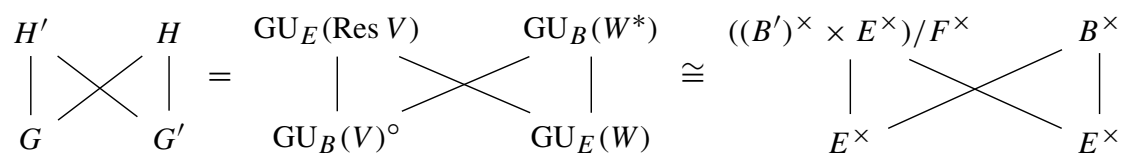


Recall from Proposition 5.14 that our choice of splittings

$$
s: \mathcal{G}_{G \times H}(\mathbb{A}) \rightarrow \mathbb{C}^{1}, \quad s^{\prime}: \mathcal{G}_{G^{\prime} \times H^{\prime}}(\mathbb{A}) \rightarrow \mathbb{C}^{1}
$$

enjoys the property that for $(\alpha, \beta) \in \mathcal{G}_{G \times G^{\prime}}(\mathbb{A})$,

$$
s^{\prime}(\alpha, \beta)=\xi(\alpha) \cdot \xi^{\prime}(\beta) \cdot s(\alpha, \beta) .
$$

THEOREM 6.16. For any Hecke characters $\chi$ and $\chi^{\prime}$ of $E$,

$$
\left\langle\theta_{\varphi}(\chi \cdot \xi), \overline{\chi^{\prime}}\right\rangle_{G^{\prime}}=\left\langle\chi, \theta_{\varphi}^{\prime}\left(\overline{\chi^{\prime} \cdot \xi^{\prime-1}}\right)\right\rangle_{G} .
$$

Proof. Unwinding definitions and using Proposition 5.14, we have

$$
\begin{aligned}
& \left\langle\theta_{\varphi}(\chi \cdot \xi), \overline{\chi^{\prime}}\right\rangle_{G^{\prime}} \\
& =\int_{\left[G^{\prime}\right]} \theta_{\varphi}(\chi \cdot \xi)\left(g^{\prime}\right) \cdot \chi^{\prime}\left(g^{\prime}\right) d g^{\prime} \\
& =\int_{\mathcal{C}} \int_{\left[G_{1}^{\prime}\right]} \theta_{\varphi}(\chi \cdot \xi)\left(g_{1}^{\prime} g_{c}^{\prime}\right) \cdot \chi^{\prime}\left(g_{1}^{\prime} g_{c}^{\prime}\right) d g_{1}^{\prime} d c \\
& =\int_{\mathcal{C}} \int_{\left[G_{1}^{\prime}\right]} \int_{\left[G_{1}\right]} \Theta\left(\omega_{\psi}\left(g_{1} g_{c}, g_{1}^{\prime} g_{c}^{\prime}\right) \varphi\right) \cdot \chi\left(g_{1} g_{c}\right) \cdot \xi\left(g_{1} g_{c}\right) \cdot \chi^{\prime}\left(g_{1}^{\prime} g_{c}^{\prime}\right) d g_{1} d g_{1}^{\prime} d c \\
& =\int_{\mathcal{C}} \int_{\left[G_{1}\right]} \int_{\left[G_{1}^{\prime}\right]} \chi\left(g_{1} g_{c}\right) \Theta\left(\omega_{\psi}^{\prime}\left(g_{1} g_{c}, g_{1}^{\prime} g_{c}^{\prime}\right) \varphi\right) \cdot \xi^{\prime}\left(g_{1}^{\prime} g_{c}^{\prime}\right)^{-1} \cdot \chi^{\prime}\left(g_{1}^{\prime} g_{c}^{\prime}\right) d g_{1}^{\prime} d g_{1} d c \\
& =\int_{\mathcal{C}} \int_{\left[G_{1}\right]} \chi\left(g_{1} g_{c}\right) \overline{\theta_{\varphi}^{\prime}\left(\overline{\chi^{\prime} \cdot \xi^{\prime-1}}\right)}\left(g_{1} g_{c}\right) d g_{1} d c \\
& =\left\langle\chi, \theta_{\varphi}^{\prime}\left(\overline{\chi^{\prime} \cdot \xi^{\prime-1}}\right)\right\rangle_{G} \text {. }
\end{aligned}
$$

Combining Theorems 6.15 and 6.16, we obtain the following result.

THEOREM 6.17. Let $\chi, \chi^{\prime}$ be Hecke characters of $E$ and let $\varphi \in \mathcal{S}(\mathbb{X}(\mathbb{A}))$. Then

$$
f_{\chi}^{B}:=\theta_{\varphi}(\chi \cdot \xi) \in \pi_{\chi}^{B}, \quad f_{\chi^{\prime}}^{B^{\prime}}:=\overline{\theta_{\varphi}^{\prime}\left(\overline{\chi^{\prime} \cdot \xi^{\prime-1}}\right)} \in \pi_{\chi^{\prime}}^{B^{\prime}},
$$

and we have

$$
\int_{\mathbb{A}_{F}^{\times} E^{\times} \backslash \mathbb{A}_{E}^{\times}} f_{\chi}^{B}(g) \cdot \chi^{\prime}(g) d g=\int_{\mathbb{A}_{F}^{\times} E^{\times} \backslash \mathbb{A}_{E}^{\times}} \chi(g) \cdot f_{\chi^{\prime}}^{B^{\prime}}(g) d g .
$$

We point out that by Theorem 6.17, if $f_{\chi}^{B}$ is a test vector for the Waldspurger torus period of $\pi_{\chi}^{B}$ with $\chi^{\prime}$, then $f_{\chi^{\prime}}^{B^{\prime}}$ is a test vector for the Waldspurger torus period of $\pi_{\chi^{\prime}}^{B^{\prime}}$ with $\chi$. 


\section{Special vectors in the Weil representation}

Recall that $F$ is a totally real field and $E=F(\mathbf{i})$ is a CM extension of $F$. We choose the trace-free element $\mathbf{i} \in E$ so that $u=\mathbf{i}^{2} \in F$ has the property that for any finite place $v$ of $F$,

$$
\operatorname{val}_{v}(u)= \begin{cases}0 & \text { if } E_{v} / F_{v} \text { is unramified } \\ 1 & \text { if } E_{v} / F_{v} \text { is ramified }\end{cases}
$$

For the rest of the paper, we take $\psi$ to be the standard additive character of $F \backslash \mathbb{A}_{F}$ (see Section 2.1). Recall that if $v$ is a finite place of $F$, then $\psi_{v}$ is trivial on $\pi_{v}^{-d_{v}} \mathcal{O}_{v}$ but nontrivial on $\pi_{v}^{-d_{v}-1} \mathcal{O}_{v}$. Furthermore, recall that we let $d x$ be the additive Haar measure on $\mathbb{A}_{F}$ self-dual with respect to $\psi$ and that $\operatorname{vol}\left(\mathcal{O}_{F_{v}}, d x_{v}\right)=q_{v}^{-d_{v} / 2}$.

In this section, we will explicitly realize the positive-weight Hecke eigenforms as theta lifts. More precisely, let $\eta_{1}, \ldots, \eta_{n}$ be the real embeddings of $F$. For any $n$-tuple of nonnegative integers $l=\left(l_{1}, \ldots, l_{n}\right)$ and any $n$-tuple of integers $k=\left(k_{1}, \ldots, k_{n}\right)$, we will specify a Schwartz function $\phi_{l}^{\prime}$ such that if $\chi_{\eta_{i}}(z)=z^{-k_{i}}$ for $i=1, \ldots, n$, then the theta lift $\theta_{\phi_{1}^{\prime}}(\chi \xi)$ is a Hecke eigenform whose weight at each infinite place $\eta_{i}$ is $\left|k_{i}\right|+1+2 l_{i}$. Note that by construction (Section 6.1), negative-weight Hecke eigenforms are not theta lifts since they are not supported on $\mathrm{GL}_{2}(F) \mathrm{GL}_{2}\left(\mathbb{A}_{F}\right)^{+}$.

Fix a place $v$ of $F$. In this section, we work place by place, and drop the subscript $v$ throughout. Let $\mathbf{W}$ be a 2-dimensional $E$-vector space endowed with the skew-Hermitian form

$$
\left\langle\left(x_{1}, x_{2}\right),\left(y_{1}, y_{2}\right)\right\rangle=\bar{x}_{1} y_{2}-\bar{x}_{2} y_{1}
$$

with respect to a fixed basis $w_{1}, w_{2}$ of $\mathbf{W}$. Let $\mathbf{V}$ be a 1-dimensional $E$-vector space endowed with the Hermitian form $(\alpha, \beta)=\alpha \bar{\beta}$. Setting $\mathbf{W}_{i}=\operatorname{span}_{\mathbb{C}}\left(w_{i}\right)$ for $i=1,2$, we have a decomposition $\mathbf{W}=\mathbf{W}_{1}+\mathbf{W}_{2}$ of $\mathbf{W}$ into maximal isotropic subspaces, and this induces a complete polarization of $\mathbb{V}$ given by

$$
\mathbb{V}=\mathbb{X}^{\prime}+\mathbb{Y}^{\prime}, \quad \mathbb{X}^{\prime}=\mathbf{V} \otimes \mathbf{W}_{1}, \quad \mathbb{Y}^{\prime}=\mathbf{V} \otimes \mathbf{W}_{2} .
$$

Fix a splitting

$$
\text { s: } \mathrm{G}(\mathrm{U}(\mathbf{V}) \times \mathrm{U}(\mathbf{W})) \rightarrow \mathbb{C}^{1}
$$

of the cocycle $z_{\mathbb{Y}^{\prime}}$ with respect to the map

$$
\iota: \mathrm{G}(\mathrm{U}(\mathbf{V}) \times \mathrm{U}(\mathbf{W})) \rightarrow \mathrm{Sp}(\mathbb{V}), \quad(h, g) \mapsto\left(v \otimes w \mapsto h^{-1} v \otimes w g\right) .
$$

This determines a homomorphism

$$
\tilde{\iota}: \mathrm{G}(\mathrm{U}(\mathbf{V}) \times \mathrm{U}(\mathbf{W})) \rightarrow \mathrm{Mp}(\mathbb{V})_{\mathbb{Y}^{\prime}}, \quad(h, g) \mapsto(\iota(h, g), \mathbf{s}(h, g)) .
$$


Recall from Equation (6.12) and Lemma 5.17 that for $\phi \in \mathcal{S}\left(\mathbb{X}^{\prime}\right)$ and $(h, g) \in$ $\mathrm{G}(\mathrm{U}(\mathbf{V}) \times \mathrm{U}(\mathbf{W}))$,

$$
\omega_{\psi}(h, g) \phi(x)=\xi^{-1}(h)|h|^{-1 / 2}\left(\omega_{\psi}\left(d\left(v(g)^{-1}\right) g\right) \phi\right)\left(x h^{-1}\right) .
$$

One can choose a basis of $\mathbb{X}^{\prime}$ and $\mathbb{Y}^{\prime}$ so that

$$
\begin{gathered}
\iota(D(a))=\left(\begin{array}{cccc}
a & & & \\
& a & & \\
& & a^{-1} & \\
& & a^{-1}
\end{array}\right), \quad \iota\left(U\left(a^{\prime}\right)\right)=\left(\begin{array}{llll}
1 & & a^{\prime} & \\
& 1 & & a^{\prime} \\
& & 1 & \\
& & & 1
\end{array}\right), \\
\iota(W)=\left(\begin{array}{llll} 
& & 1 & \\
& & & 1 \\
-1 & & & \\
& -1 &
\end{array}\right) .
\end{gathered}
$$

By the computations of Section 5.6 and Equations (3.1)-(3.3),

$$
\begin{aligned}
\omega_{\psi}(1, D(a)) \varphi(x) & =\xi(a)^{-1} \cdot|\operatorname{det} a| \cdot \varphi(x a) \\
\omega_{\psi}\left(1, U\left(a^{\prime}\right)\right) \varphi(x) & =\psi\left(\frac{1}{4} \operatorname{Tr}_{E / F}\left(a^{\prime} x \bar{x}\right)\right) \cdot \varphi(x) \\
\omega_{\psi}(1, W) \varphi(x) & =(u,-1)_{F} \cdot \gamma_{F}\left(u, \frac{1}{2} \psi\right) \cdot \int_{F^{2}} \varphi(y) \psi\left(\frac{1}{2} \operatorname{Tr}_{E / F}(x \bar{y})\right) d y
\end{aligned}
$$

If $v$ is a finite place, let $c\left(\pi_{\chi}\right)$ be the conductor of $\pi_{\chi}$ and let $K_{0}^{\prime}(N)$ be the compact open subgroup as defined in Section 2.2. Writing $d(v)=\left(\begin{array}{ll}1 & 0 \\ 0 & v\end{array}\right) \in \mathrm{GL}_{2}(F)$ for $v \in F^{\times}$, define

$$
K_{0}(N):= \begin{cases}K_{0}^{\prime}(N) & \text { if } F \text { has odd residue characteristic } \\ d(2) K_{0}^{\prime}(N) d(1 / 2) & \text { if } F \text { has even residue characteristic }\end{cases}
$$

7.1. Schwartz functions. In this section, we introduce Schwartz functions that transform nicely under the Weil representation. These functions have been considered in various places before. At the finite places, they have appeared for example in [P06, Proposition 2.5.1], [X07, N1]. At the infinite places, our choice is constructed from a confluent hypergeometric function ${ }_{1} F_{1}(a, b, t)$ of the first type. This is related to the role of hypergeometric functions in matrix coefficients of representations of $\mathrm{SL}_{2}(\mathbb{R})$ (see, for example, [X07, Appendix], [VK91, Ch. 6, 7]).

7.1.1. Infinite places. In this section, let $v$ be an infinite place of $F$. 
Definition 7.1. For $k \in \mathbb{Z}$ and $l \in \mathbb{Z}_{\geqslant 0}$, define

$$
\phi_{k, l}^{\prime}(z):= \begin{cases}{ }_{1} F_{1}(-l, k+1,4 \pi z \bar{z}) \bar{z}^{k} e^{-2 \pi z \bar{z}} & \text { if } k \geqslant 0, \\ { }_{1} F_{1}(-l,-k+1,4 \pi z \bar{z}) z^{-k} e^{-2 \pi z \bar{z}} & \text { if } k<0,\end{cases}
$$

where ${ }_{1} F_{1}(a, b, t)$ is the Kummer confluent hypergeometric function for constants $a, b$

$$
\begin{aligned}
{ }_{1} F_{1}(a, b, t):= & \sum_{j=0}^{\infty} \frac{(a)_{j}}{(b)_{j}} \frac{1}{j !} t^{j}, \quad \text { where }(a)_{0}:=1, \\
& \text { and }(a)_{j}:=a(a+1)(a+2) \cdots(a+j-1) .
\end{aligned}
$$

Observe that ${ }_{1} F_{1}(a, b, t)$ is entire in $t$ so long as $b \notin \mathbb{Z}_{\leqslant 0}$, so that in particular, $\phi_{k, l}^{\prime}$ is entire for all $k \in \mathbb{Z}$ and $l \in \mathbb{Z}_{\geqslant 0}$.

The following lemma is well known.

LEMMA 7.2. (a) The function ${ }_{1} F_{1}(a, b, t)$ is a solution to the differential equation

$$
t f^{\prime \prime}(t)+(b-t) f^{\prime}(t)-a f(t)=0 .
$$

(b) If $\operatorname{Re}(\alpha)>0$ and $\operatorname{Re}(c)>0$, then

$$
\int_{0}^{\infty} t^{\alpha-1} e^{-c t} F_{1}(a, b,-t) d t=c^{-\alpha} \Gamma(\alpha)_{2} F_{1}\left(a, \alpha, b,-\frac{1}{c}\right),
$$

where

$$
{ }_{2} F_{1}\left(a, \alpha, b,-\frac{1}{c}\right)=\sum_{j=0}^{\infty} \frac{(a)_{j}(\alpha)_{j}}{(b)_{j}} \frac{1}{j !}\left(-\frac{1}{c}\right)^{j} .
$$

LEMMA 7.3. For $\alpha \in \mathbb{C}^{1}$ and $r(\theta)=\left(\begin{array}{cc}\cos (\theta) & \sin (\theta) \\ -\sin (\theta) & \cos (\theta)\end{array}\right) \in \mathrm{SO}(2)$,

$$
\omega_{\psi}(\alpha, r(\theta)) \phi_{k, l}^{\prime}=\xi\left(\alpha^{-1}\right) \alpha^{-k} e^{i(|k|+1+2 l) \theta} \phi_{k, l}^{\prime} .
$$

Proof. We follow a similar proof strategy to [X07, Proposition 2.2.5]. We compute on the Lie algebra $\mathfrak{s l}_{2}(\mathbb{R})$. It is well known that for $X_{+}=\left(\begin{array}{ll}0 & 1 \\ 0 & 0\end{array}\right)$, $X_{-}=\left(\begin{array}{ll}0 & 0 \\ 1 & 0\end{array}\right)$,

$$
\omega_{\psi}\left(X_{+}\right) \phi=2 \pi i z \bar{z} \phi, \quad \omega_{\psi}\left(X_{-}\right) \phi=-\frac{1}{2 \pi i} \frac{\partial}{\partial z}\left(\frac{\partial}{\partial \bar{z}} \phi\right) .
$$

We first handle the case $k \geqslant 0$. For any doubly differentiable function $f$ satisfying the differential equation

$$
t f^{\prime \prime}(t)+(k+1-t) f^{\prime}(t)=-l f(t)
$$


we have, following from a long calculus computation,

$$
\begin{aligned}
\omega_{\psi}\left(X_{+}-X_{-}\right)\left(f(4 \pi z \bar{z}) \bar{z}^{k} e^{-2 \pi z \bar{z}}\right) \\
=i\left[(k+1) f(4 \pi z \bar{z})-2\left((k+1-4 \pi z \bar{z}) f^{\prime}(4 \pi z \bar{z})\right.\right. \\
\left.\left.\quad+4 \pi z \bar{z} f^{\prime \prime}(4 \pi z \bar{z})\right)\right] \bar{z}^{k} e^{-2 \pi z \bar{z}} \\
=i(k+1+2 l) f(4 \pi z \bar{z}) \bar{z}^{k} e^{-2 \pi z \bar{z}} .
\end{aligned}
$$

By Lemma 7.2(a), ${ }_{1} F_{1}(-l, k+1, t)$ is such an $f(t)$ and hence the desired conclusion follows.

Now assume $k<0$. For any doubly differentiable function $f$ satisfying the differential equation

$$
t f^{\prime \prime}(t)+(-k+1-t) f^{\prime}(t)=-l f(t)
$$

we have

$$
\begin{aligned}
\omega_{\psi}\left(X_{+}-X_{-}\right)\left(f(4 \pi z \bar{z}) z^{-k} e^{-2 \pi z \bar{z}}\right) \\
=i\left[(-k+1) f(4 \pi z \bar{z})-2\left((-k+1-4 \pi z \bar{z}) f^{\prime}(4 \pi z \bar{z})\right.\right. \\
\left.\left.\quad+4 \pi z \bar{z} f^{\prime \prime}(4 \pi z \bar{z})\right)\right] z^{-k} e^{-2 \pi z \bar{z}} \\
=i(-k+1+2 l) f(4 \pi z \bar{z}) z^{-k} e^{-2 \pi z \bar{z}} .
\end{aligned}
$$

By Lemma $7.2(\mathrm{a}),{ }_{1} F_{1}(-l,-k+1, t)$ is such an $f(t)$, and so the desired conclusion follows.

Finally, it is easy to see that $\omega_{\psi}(\alpha, 1) \phi_{k, l}^{\prime}=\xi\left(\alpha^{-1}\right) \alpha^{-k} \phi_{k, l}^{\prime}$, and it follows that

$$
\omega_{\psi}(\alpha, r(\theta)) \phi_{k, l}^{\prime}=\xi\left(\alpha^{-1}\right) \alpha^{-k} e^{-(|k|+1+2 l) \theta} \phi_{k, l}^{\prime} .
$$

The following lemma is useful in understanding the relationship between the $\phi_{k, l}^{\prime}$ with respect to the Maass-Shimura operator on modular forms.

LemMA 7.4. Write $z=x+y i \in \mathbb{C}$. For $y \neq 0$, we have

$$
\delta_{|k|+1}^{l}\left(y^{1 / 2} e^{2 \pi i x v \bar{v}} \phi_{k, 0}^{\prime}(v \sqrt{y})\right)=\frac{(|k|+1)_{l}}{(2 \pi i)^{l}(2 i)^{l}} \cdot\left(y^{-l+1 / 2} e^{2 \pi i x v \bar{v}} \phi_{k, l}^{\prime}(v \sqrt{y})\right) .
$$

Proof. This amounts to showing

$$
\begin{aligned}
& \left(\frac{\partial}{\partial z}+\frac{|k|+1+2 l}{z-\bar{z}}\right)\left[y^{-l+1 / 2} e^{2 \pi i x v \bar{v}} \phi_{k, 0}^{\prime}(v \sqrt{y})\right] \\
& =\frac{|k|+1+l}{2 i} \cdot\left(y^{-l-1+1 / 2} e^{2 \pi i x v \bar{v}} \phi_{k, l+1}^{\prime}(v \sqrt{y})\right) .
\end{aligned}
$$


Unwinding definitions, this amounts to showing

$$
\begin{aligned}
& \left(\frac{\partial}{\partial z}+\frac{|k|+1+2 l}{z-\bar{z}}\right)\left(y_{1}^{-l} F_{1}(-l,|k|+1,4 \pi v \bar{v} y) e^{2 \pi i v \bar{v} z}\right) \\
& \quad=\frac{|k|+1+l}{2 i} \cdot\left(y^{-l-1}{ }_{1} F_{1}(-l-1,|k|+1,4 \pi v \bar{v} y) e^{2 \pi i v \bar{v} z}\right) .
\end{aligned}
$$

Verifying this is a straightforward calculation. For example, the coefficient of $y^{-l-1}$ on the left-hand side is equal to $\left(\frac{-l}{2 i}+\frac{|k|+1+2 l}{2 i}\right) \cdot e^{2 \pi i v \bar{v} z}$, and this agrees with the right-hand side.

7.1.2. Finite nonsplit places. In this section, let $v$ be a finite nonsplit place of $F$ lying under a single prime $w$ of $E$. Then $E_{w}$ is a field and $E_{w} / F_{v}$ is either unramified or ramified. Assume that $E_{w}, F_{v}$ have odd residue characteristic. We drop the subscripts $w$ and $v$ throughout this section.

DEFINITION 7.5. Define

$$
\phi^{\prime}(x):= \begin{cases}\mathbb{1}_{\mathcal{O}_{E}}(x) & \text { if } \chi \text { is unramified } \\ \chi(x) \mathbb{1}_{\mathcal{O}_{E}^{\times}}(x) & \text { otherwise }\end{cases}
$$

LEMMA 7.6. Let $\psi^{\prime}$ be an unramified nontrivial additive character of $F$. For $h \in \mathcal{O}_{E}^{\times}$and $g=\left(\begin{array}{ll}a & b \\ c & d\end{array}\right) \in K_{0}:=K_{0}\left(c\left(\rho_{\chi}\right)\right)$ such that $\operatorname{Nm}(h)=\operatorname{det}(g)$, we have

$$
\omega_{\psi^{\prime}}(h, g) \phi^{\prime}=(\chi \xi)^{-1}(h) \cdot\left(\chi \epsilon_{E / F}\right)(a) \cdot \phi^{\prime} .
$$

Proof. See [X07, Proposition 2.2.4], [P06, Proposition 2.5.1], and [Ch18, Lemma 8.6].

LEMMA 7.7. For $h \in \mathcal{O}_{E}^{\times}$and $g=\left(\begin{array}{ll}a & b \\ c & d\end{array}\right)$ such that $\operatorname{Nm}(h)=\operatorname{det}(g)$, we have

$$
\omega_{\psi}\left(h, d(\delta)^{-1} g d(\delta)\right) \phi^{\prime}=(\chi \xi)^{-1}(h) \cdot\left(\chi \epsilon_{E / F}\right)(a) \cdot \phi^{\prime} .
$$

Proof. Since $\psi$ has conductor $\delta, \psi^{\prime}(x):=\psi(\delta x)$ is an unramified nontrivial additive character of $F$. The conclusion follows by Equation (3.4) and Lemma 7.6.

7.1.3. Finite split places. In this section, we let $v$ be a finite split place of $F$. Then $E_{v} \cong F_{v} \oplus F_{v}$. We drop the subscript $v$ throughout this section. 
DEFINITION 7.8. For a character $\chi=\chi_{1} \otimes \chi_{2}: F^{\times} \times F^{\times} \rightarrow \mathbb{C}^{\times}$, define

$$
\phi^{\prime}\left(x_{1}, x_{2}\right):= \begin{cases}\mathbb{1}_{\mathcal{O}_{F}}\left(x_{1}\right) \mathbb{1}_{\mathcal{O}_{F}}\left(x_{2}\right) & \text { if } \chi \text { is unramified, } \\ \chi\left(x_{1}, x_{2}\right) \mathbb{1}_{\mathcal{O}_{F}^{\times}}\left(x_{1}\right) \mathbb{1}_{\mathcal{O}_{F}^{\times}}\left(x_{2}\right) & \text { otherwise. }\end{cases}
$$

LEMMA 7.9. Let $\psi^{\prime}$ be an unramified nontrivial additive character of F. For $h \in \mathcal{O}_{F}^{\times} \times \mathcal{O}_{F}^{\times}$and $g=\left(\begin{array}{ll}a & b \\ c & d\end{array}\right) \in K_{0}$ with $\operatorname{Nm}(h)=\operatorname{det}(g)$, we have

$$
\omega_{\psi^{\prime}}(h, g) \phi^{\prime}=(\chi \xi)^{-1}(h) \cdot \chi_{1}(a) \chi_{2}(a) \cdot \phi^{\prime} .
$$

Proof. See [X07, Proposition 2.2.4], [P06, Proposition 2.5.1], and [Ch18, Lemma 8.9].

By the same argument as in Lemma 7.7, we have the following lemma.

LEMMA 7.10. For $h \in \mathcal{O}_{F}^{\times} \times \mathcal{O}_{F}^{\times}$and $g=\left(\begin{array}{ll}a & b \\ c & d\end{array}\right) \in K_{0}$ with $\mathrm{Nm}(h)=\operatorname{det}(g)$, we have

$$
\omega_{\psi}\left(h, d(\delta)^{-1} g d(\delta)\right) \phi^{\prime}=(\chi \xi)^{-1}(h) \cdot \chi_{1}(a) \chi_{2}(a) \cdot \phi^{\prime} .
$$

7.2. Local zeta integrals. In this section, we calculate the local zeta integrals $Z\left(\frac{1}{2}, \Phi_{v}, \chi_{v}\right)$ for the Siegel-Weil section $\Phi_{v}=\Phi_{v}^{\mathrm{O}, \mathrm{Sp}}\left(\delta\left(\phi_{v}^{\prime} \otimes \overline{\phi_{v}^{\prime}}\right)\right)$ (see Section 6.2).

7.2.1. Infinite nonsplit places. Let $v$ be an infinite nonsplit place. We say that $\chi_{v}$ has infinity type $\left(k_{1}, k_{2}\right)$ if

$$
\chi_{v}: \mathbb{C}^{\times} \rightarrow \mathbb{C}^{\times}, \quad z \mapsto z^{-k_{1}} \bar{z}^{-k_{2}} .
$$

Assume that $\chi_{v}(z)=z^{k}$ for $z \in \mathbb{C}^{1}$, so that either $\chi_{v}$ is of type $(-k+j, j)$ or $(-j, k-j)$ for some integer $j$. Pick an integer $l \in \mathbb{Z}_{\geqslant 0}$ and take

$$
\phi_{v}^{\prime}(z):=\phi_{k, l}^{\prime}(z)= \begin{cases}{ }_{1} F_{1}(-l, k+1,4 \pi z \bar{z}) \bar{z}^{k} e^{-2 \pi z \bar{z}} & \text { if } k \geqslant 0, \\ { }_{1} F_{1}(-l,-k+1,4 \pi z \bar{z}) z^{-k} e^{-2 \pi z \bar{z}} & \text { if } k<0 .\end{cases}
$$

LEMMA 7.11. Let $v$ be an infinite nonsplit place. Then

$$
Z_{v}\left(\frac{1}{2}, \Phi_{v}, \chi_{v}\right)=\operatorname{vol}\left(\mathbb{C}^{1}\right)\left\langle\phi^{\prime}, \phi^{\prime}\right\rangle=\frac{(2 \pi)^{2}}{4^{|k|+1} \pi^{|k|+1}} \cdot \frac{l !(|k|) !^{2}}{(l+|k|) !} .
$$

Proof. By Lemma 7.3, $\omega_{\psi}(\alpha, 1) \phi_{v}^{\prime}=\xi\left(\alpha^{-1}\right) \alpha^{-k} \phi_{v}^{\prime}$. Thus

$$
\begin{aligned}
Z_{v}\left(\frac{1}{2}, \Phi_{v}, \chi_{v}\right) & =\int_{\mathbb{C}^{1}}\left\langle\omega_{\psi}(g, 1) \phi_{v}^{\prime}, \phi_{v}^{\prime}\right\rangle\left(\chi_{v} \xi_{v}\right)(g) d g \\
& =\operatorname{vol}\left(\mathbb{C}^{1}\right)\left\langle\phi_{v}^{\prime}, \phi_{v}^{\prime}\right\rangle=\pi^{-1}\left\langle\phi_{v}^{\prime}, \phi_{v}^{\prime}\right\rangle .
\end{aligned}
$$


We have

$$
\begin{aligned}
\left\langle\phi_{v}^{\prime}, \phi_{v}^{\prime}\right\rangle & =\int_{\mathbb{C}}{ }_{1} F_{1}(-l,|k|+1,4 \pi z \bar{z})^{2} \cdot(z \bar{z})^{|k|} \cdot e^{-4 \pi z \bar{z}} d z d \bar{z} \\
& =\frac{2 \pi}{(4 \pi)(4 \pi)^{|k|}} \int_{0}^{\infty}{ }_{1} F_{1}(-l,|k|+1, t)^{2} \cdot t^{|k|} \cdot e^{-t} d t \\
& =\frac{2 \pi}{(4 \pi)^{|k|+1}} \frac{l !(|k|) !^{2}}{(l+|k|) !}=\frac{2 \pi}{(4 \pi)^{|k|+1}} \frac{(|k|) !}{\left(\begin{array}{c}
l+|k| \\
|k|
\end{array}\right)}
\end{aligned}
$$

7.2.2. Finite nonsplit places. Recall from Section 7 that we set

$$
\phi_{v}^{\prime}(x)= \begin{cases}\mathbb{1}_{\mathcal{O}_{E_{v}}}(x) & \text { if } \chi_{v} \text { is unramified } \\ \chi_{v}(x) \mathbb{1}_{\mathcal{O}_{E_{v}}^{\times}}(x) & \text { if } \chi_{v} \text { is ramified }\end{cases}
$$

LEMMA 7.12. Let $v$ be a finite nonsplit place. If $E_{v} / F_{v}$ is unramified, then

$$
\begin{aligned}
& Z_{v}\left(\frac{1}{2}, \Phi_{v}, \chi_{v}\right) \\
& \quad= \begin{cases}q_{v}^{-d_{v} / 2} & \text { if } E_{v} / F_{v} \text { is unramified and } \chi_{v} \text { is unramified, } \\
q_{v}^{-d_{v} / 2}\left(1-q_{v}^{-2}\right) & \text { if } E_{v} / F_{v} \text { is unramified and } \chi_{v} \text { is ramified, } \\
q_{v}^{-1} q_{v}^{-d_{v} / 2} & \text { if } E_{v} / F_{v} \text { is ramified and } \chi_{v} \text { is unramified, } \\
q_{v}^{-1} q_{v}^{-d_{v} / 2}\left(1-q_{v}^{-1}\right) & \text { if } E_{v} / F_{v} \text { is ramified and } \chi_{v} \text { is ramified. }\end{cases}
\end{aligned}
$$

Proof. By Lemma 7.7, for $g \in E_{v}^{1}$, we have $\omega_{\psi}(g, 1) \phi^{\prime}=\left(\chi_{v} \xi_{v}\right)^{-1}(g) \cdot \phi^{\prime}$. This implies that

$$
\begin{aligned}
Z_{v}\left(\frac{1}{2}, \Phi_{v}, \chi_{v}\right) & =\operatorname{vol}\left(E_{v}^{1}, d^{1} x_{v}^{\mathrm{Tam}}\right) \int_{E_{v}^{1}}\left\langle\omega_{\psi}(g, 1) \phi^{\prime}, \phi^{\prime}\right\rangle\left(\chi \xi_{\mathbb{Y}^{\prime}}\right)_{v}(g) d g \\
& =\operatorname{vol}\left(E_{v}^{1}, d^{1} x_{v}^{\mathrm{Tam}}\right)^{2}\left\langle\phi^{\prime}, \phi^{\prime}\right\rangle \\
& = \begin{cases}\operatorname{vol}\left(E_{v}^{1}, d^{1} x_{v}^{\mathrm{Tam}}\right)^{2} \operatorname{vol}\left(\mathcal{O}_{E_{v}}, d x_{v}\right) & \text { if } \chi_{v} \text { is unramified } \\
\operatorname{vol}\left(E_{v}^{1}, d^{1} x_{v}^{\mathrm{Tam}}\right)^{2} \operatorname{vol}\left(\mathcal{O}_{E_{v}}^{\times}, d x_{v}\right) & \text { otherwise. }\end{cases}
\end{aligned}
$$

The desired conclusion now follows from the measures in Section (2.1).

7.2.3. Finite split places. Let $v$ be a finite split place and write $\chi_{v}=\chi_{1, v} \otimes$ $\chi_{2, v}: F_{v}^{\times} \times F_{v}^{\times} \rightarrow \mathbb{C}^{\times}$. Recall that

$$
\phi^{\prime}\left(x_{1}, x_{2}\right):= \begin{cases}\mathbb{1}_{\mathcal{O}_{F_{v}}}\left(x_{1}\right) \mathbb{1}_{\mathcal{O}_{F_{v}}}\left(x_{2}\right) & \text { if } \chi_{v} \text { is unramified } \\ \chi_{v}\left(x_{1}, x_{2}\right) \mathbb{1}_{\mathcal{O}_{F_{v}}^{\times}}\left(x_{1}\right) \mathbb{1}_{\mathcal{O}_{F_{v}}^{\times}}\left(x_{2}\right) & \text { otherwise. }\end{cases}
$$


LEMMA 7.13. Let $v$ be a finite split place and assume that $\chi_{v}$ is unramified. Then

$$
Z_{v}\left(\frac{1}{2}, \Phi_{v}, \chi_{v}\right)=q_{v}^{-3 d_{v} / 2} \cdot \frac{L_{v}\left(1, \chi_{1, v} \otimes \chi_{2, v}^{-1}\right) L_{v}\left(1, \chi_{1, v}^{-1} \otimes \chi_{2, v}\right)}{L_{v}\left(2, \varepsilon_{E / F}\right)}
$$

Proof. In this setting, $E_{v}^{1}=\left\{\left(a, a^{-1}\right) \in F_{v}^{\times} \times F_{v}^{\times}\right\}$. By Lemma 5.17,

$$
\begin{aligned}
\omega_{\psi}\left(\left(a, a^{-1}\right), 1\right) \phi^{\prime}\left(x_{1}, x_{2}\right) & =\xi_{v}\left(a, a^{-1}\right)^{-1} \phi^{\prime}\left(x_{1} a^{-1}, x_{2} a\right) \\
& =\xi_{v}\left(a, a^{-1}\right)^{-1} \mathbb{1}_{a \mathcal{O}_{F_{v}}}\left(x_{1}\right) \mathbb{1}_{a^{-1} \mathcal{O}_{F_{v}}}\left(x_{2}\right) .
\end{aligned}
$$

Hence

$$
\begin{aligned}
& \left\langle\omega_{\psi}\left(\left(a, a^{-1}\right), 1\right) \phi^{\prime}, \phi^{\prime}\right\rangle \\
& =\int_{\mathbb{X}_{v}^{\prime}} \xi_{v}\left(a, a^{-1}\right)^{-1} \mathbb{1}_{a \mathcal{O}_{F_{v}}}\left(x_{1}\right) \mathbb{1}_{a^{-1} \mathcal{O}_{F_{v}}}\left(x_{2}\right) \mathbb{1}_{\mathcal{O}_{F_{v}}}\left(x_{1}\right) \mathbb{1}_{\mathcal{O}_{F_{v}}}\left(x_{2}\right) d x_{1} d x_{2} \\
& =\xi_{v}\left(a, a^{-1}\right)^{-1} \operatorname{vol}\left(a \mathcal{O}_{F_{v}} \cap \mathcal{O}_{F_{v}}, d x_{v}\right) \operatorname{vol}\left(a^{-1} \mathcal{O}_{F_{v}} \cap \mathcal{O}_{F_{v}}, d x_{v}\right) \\
& =\xi_{v}\left(a, a^{-1}\right)^{-1} \frac{1}{q_{v}^{|\operatorname{val}(a)|}} \operatorname{vol}\left(\mathcal{O}_{F_{v}}, d x_{v}\right)^{2}=\xi_{v}\left(a, a^{-1}\right)^{-1} \frac{1}{q_{v}^{|\operatorname{val}(a)|}} q_{v}^{-d_{v}} .
\end{aligned}
$$

We therefore have, writing $\pi=\pi_{v}$ for a uniformizer of $F_{v}$,

$$
\begin{aligned}
& Z_{v}\left(\frac{1}{2}, \Phi_{v}, \chi_{v}\right) \\
& \quad=\int_{F_{v}^{\times}}\left\langle\omega_{\psi}\left(a, a^{-1}\right) \phi_{v}^{\prime}, \phi_{v}^{\prime}\right\rangle \xi_{v}\left(a, a^{-1}\right) \chi_{v}\left(a, a^{-1}\right) d a \\
& \quad=\sum_{n \in \mathbb{Z}} \int_{\mathcal{O}_{F_{v}}^{\times}}\left\langle\omega_{\psi}\left(\pi^{n} a, \pi^{-n} a^{-1}\right) \phi_{v}^{\prime}, \phi_{v}^{\prime}\right\rangle \xi_{v}\left(\pi^{n} a, \pi^{-n} a^{-1}\right) \chi_{v}\left(\pi^{n} a, \pi^{-n} a^{-1}\right) d a \\
& \quad=q_{v}^{-3 d_{v} / 2} \cdot \frac{1-q_{v}^{-2}}{\left(1-q_{v}^{-1} \chi_{v}\left(\pi^{-1}, \pi\right)\right)\left(1-q_{v}^{-1} \chi_{v}\left(\pi, \pi^{-1}\right)\right)} \\
& \quad=q_{v}^{-3 d_{v} / 2} \cdot \frac{L_{v}\left(1, \chi_{1, v} \otimes \chi_{2, v}^{-1}\right) L_{v}\left(1, \chi_{1, v}^{-1} \otimes \chi_{2, v}\right)}{L_{v}\left(2, \varepsilon_{E / F}\right)} .
\end{aligned}
$$

LEMMA 7.14. Let $v$ be a finite split place and assume that $\chi_{v}$ is ramified. Then

$$
Z_{v}\left(\frac{1}{2}, \Phi_{v}, \chi_{v}\right)=q_{v}^{-3 d_{v} / 2}\left(1-q_{v}^{-1}\right)^{2} .
$$

Proof. We have $\omega_{\psi}\left(\left(a, a^{-1}\right), 1\right) \phi^{\prime}\left(x_{1}, x_{2}\right)=\xi_{v}\left(a, a^{-1}\right)^{-1} \chi_{v}\left(a, a^{-1}\right)^{-1} \mathbb{1}_{a \mathcal{O}_{F_{v}}^{\times}}\left(x_{1}\right)$ $\mathbb{1}_{a^{-1} \mathcal{O}_{F_{v}}^{\times}}\left(x_{2}\right)$ so that

$$
\left\langle\omega_{\psi}\left(\left(a, a^{-1}\right), 1\right) \phi^{\prime}, \phi^{\prime}\right\rangle=\xi_{v}\left(a, a^{-1}\right)^{-1} \chi_{v}\left(a, a^{-1}\right)^{-1} \operatorname{vol}\left(\mathcal{O}_{F_{v}}^{\times}, d x_{v}\right)^{2} \mathbb{1}_{\mathcal{O}_{F_{v}}^{\times}}(a) .
$$

Thus $Z_{v}\left(\frac{1}{2}, \Phi_{v}, \chi_{v}\right)=\operatorname{vol}\left(\mathcal{O}_{F_{v}}^{\times}, d x_{v}\right)^{2} \operatorname{vol}\left(\mathcal{O}_{F_{v}}^{\times}, d^{1} x_{v}^{\mathrm{Tam}}\right)$. 


\section{An explicit Rallis inner product formula}

Let $F$ be a totally real number field and let $E / F$ be a CM extension. Assume that every place $v$ of $F$ over 2 splits in $E$. Let $\eta_{1}, \ldots, \eta_{n}$ be the real embeddings of $F$. Let $\chi: E^{\times} \backslash \mathbb{A}_{E}^{\times} \rightarrow \mathbb{C}^{\times}$be a Hecke character of infinity type $(k+j, j)$, where $k=\left(k_{1}, \ldots, k_{n}\right), j=\left(j_{1}, \ldots, j_{n}\right) \in \mathbb{Z}^{n}$. Assume that $B=M_{2}(F)$ and let $W_{0}=\operatorname{Res}_{B / E} B=W_{1}+W_{2}$ be a decomposition of the $E$-space $W_{0}$ into totally isotropic subspaces. Set $\mathbb{X}^{\prime}=\operatorname{Res}_{E / F}\left(E \otimes W_{1}\right), \mathbb{Y}^{\prime}=\operatorname{Res}_{E / F}\left(E \otimes W_{2}\right)$, and define a Schwartz function $\phi^{\prime}=\otimes_{v} \phi_{v}^{\prime} \in \mathcal{S}\left(\mathbb{X}^{\prime}(\mathbb{A})\right)$ as in Section 7:

$$
\phi_{l, v}^{\prime}(z):= \begin{cases}{ }_{1} F_{1}\left(-l_{i}, k_{i}+1,4 \pi z \bar{z}\right) \bar{z}^{k} e^{-2 \pi z \bar{z}} & \text { if } v=\eta_{i} \mid \infty \text { and } k \geqslant 0, \\ { }_{1} F_{1}\left(-l_{i},-k_{i}+1,4 \pi z \bar{z}\right) z^{-k} e^{-2 \pi z \bar{z}} & \text { if } v=\eta_{i} \mid \infty \text { and } k<0, \\ \mathbb{1}_{\mathcal{O}_{E_{v}}}(z) & \text { if } v \text { is nonsplit and } \chi_{v} \text { is unramified, } \\ \chi_{v}(z) \mathbb{1}_{\mathcal{O}_{E_{v}}^{\times}}(z) & \text { if } v \text { is nonsplit and } \chi_{v} \text { is ramified, } \\ \mathbb{1}_{\mathcal{O}_{F_{v}}\left(z_{1}\right) \mathbb{1}_{\mathcal{O}_{F_{v}}}\left(z_{2}\right)} & \text { if } v \text { splits and } \chi_{v} \text { is unramified, } \\ \chi_{v}\left(z_{1}, z_{2}\right)^{-1} \mathbb{1}_{\mathcal{O}_{F_{v}}^{\times}}\left(z_{1}\right) \mathbb{1}_{\mathcal{O}_{F_{v}}^{\times}}\left(z_{2}\right) & \text { if } v \text { splits and } \chi_{v} \text { is ramified. }\end{cases}
$$

Define

$$
\Sigma_{\chi}:=\left\{v: \chi_{v} \text { is unramified }\right\}, \quad \Sigma_{\tilde{\chi}}:=\left\{v: \tilde{\chi}_{v} \text { is unramified }\right\} .
$$

For each place $v$ of $F$, define

$$
C_{v}:= \begin{cases}\frac{(2 \pi)^{2}}{4\left|k_{i}\right|+1} \pi^{\left|k_{i}\right|+1} \cdot \frac{l_{i} !\left(\left|k_{i}\right|\right) !^{2}}{\left(l_{i}+\left|k_{i}\right|\right) !} & \text { if } v=\eta_{i} \mid \infty \\ q_{v}^{-d_{v} / 2} & \text { if } v \notin \Sigma_{\chi}, v \notin \Sigma_{\tilde{\chi}}, v \text { unram } \\ q_{v}^{-d_{v} / 2}\left(1-q_{v}^{-2}\right) & \text { if } v \in \Sigma_{\chi}, v \notin \Sigma_{\tilde{\chi}}, v \text { unram } \\ q_{v}^{-d_{v} / 2} & \text { if } v \in \Sigma_{\chi}, v \in \Sigma_{\tilde{\chi}}, v \text { unram } \\ q_{v}^{-d_{v} / 2} q_{v}^{-1}\left(1-q_{v}^{-2}\right)^{-1}\left(1-\tilde{\chi}_{w}\left(\pi_{w}\right) q_{v}^{-1}\right) & \text { if } v \notin \Sigma_{\chi}, v \notin \Sigma_{\tilde{\chi}}, v \text { ram } \\ q_{v}^{-d_{v} / 2} q_{v}^{-1}\left(1-q_{v}^{-1}\right)\left(1-q_{v}^{-2}\right)^{-1}\left(1-\tilde{\chi}_{v}\left(\pi_{v}\right) q_{v}^{-1}\right) & \text { if } v \in \Sigma_{\chi}, v \notin \Sigma_{\tilde{\chi}}, v \text { ram } \\ q_{v}^{-d_{v} / 2} q_{v}^{-1}\left(1-q_{v}^{-1}\right)\left(1-q_{v}^{-2}\right)^{-1} & \text { if } v \in \Sigma_{\chi}, v \in \Sigma_{\tilde{\chi}}, v \text { ram } \\ q_{v}^{-3 d_{v} / 2} & \text { if } v \notin \Sigma_{\chi}, v \notin \Sigma_{\tilde{\chi}}, v \text { split } \\ q_{v}^{-3 d_{v} / 2}\left(1-\left(\chi_{1, v} \chi_{2, v}^{-1}\right)\left(\pi_{v}\right) q_{v}^{-1}\right)\left(1-\left(\chi_{1, v}^{-1} \chi_{2, v}\right)\left(\pi_{v}\right) q_{v}^{-1}\right) & \text { if } v \in \Sigma_{\chi}, v \notin \Sigma_{\tilde{\chi}}, v \text { split } \\ q_{v}^{-3 d_{v} / 2}\left(1-q_{v}^{-1}\right)\left(1+q_{v}^{-1}\right)^{-1} & \text { if } v \in \Sigma_{\chi}, v \in \Sigma_{\tilde{\chi}}, v \text { split. }\end{cases}
$$

THEOREM 8.1. The Petersson inner product of the theta lift $\theta_{\phi^{\prime}}(\chi \xi)$ is

$$
\left\langle\theta_{\phi^{\prime}}(\chi \xi), \theta_{\phi^{\prime}}(\chi \xi)\right\rangle=\frac{\rho_{F}}{\rho_{E}} \cdot \frac{L(1, \tilde{\chi})}{\zeta(2)} \cdot \prod_{v} C_{v},
$$

where $C_{v}=1$ at all but finitely many places. In particular, $\theta_{\phi^{\prime}}(\xi \chi) \neq 0$ if $\chi$ is nontrivial on $\mathbb{A}_{E}^{1}$. 
Proof. By the results of Section 7.2, it is a straightforward comparison to see that

$$
Z\left(\frac{1}{2}, \Phi_{v}, \chi_{v}\right)=C_{v} \cdot \frac{L_{v}(1, \tilde{\chi})}{\zeta_{v}(2)} \text { for all places } v \text { of } F
$$

Since all but finitely many places simultaneously satisfy the conditions $d_{v}=0$, $v \notin \Sigma_{\chi}, v \notin \Sigma_{\tilde{\chi}}$, and $v$ is split or unramified, we see that $C_{v}=1$ for all but finitely many places, and the desired equation now follows from the doubling method. Observe that the factor $\rho_{F} / \rho_{E}$ comes from the definition of the Tamagawa measure on $\mathbb{A}_{E}^{1}$ and the local measures on $E_{v}^{1}$ (Section 2.1).

Finally, since $C_{v} \neq 0$ for all $v$, it follows that $\theta_{\phi^{\prime}}(\chi \xi) \neq 0$ if and only if $L(1$, $\tilde{\chi}) \neq 0$. But $L(1, \tilde{\chi}) \neq 0$ if and only if $\chi$ is trivial on $\mathbb{A}_{E}^{1}$, so the final assertion holds.

The Shimura-Maass differential operator

$$
\delta_{k}:=\frac{1}{2 \pi i}\left(\frac{\partial}{\partial z}+\frac{k}{z-\bar{z}}\right)
$$

maps real analytic modular forms of weight $k$ to real analytic modular forms of weight $k+2$. Define the composite operator

$$
\delta_{k}^{l}:=\delta_{k+2 l-2} \circ \cdots \circ \delta_{k+2} \circ \delta_{k}
$$

mapping real analytic modular forms of weight $k$ to real analytic modular forms of weight $k+2 l$.

Let $f_{\chi}$ be the normalized newform of weight

$$
|k|+1=\left(\left|k_{1}\right|+1, \ldots,\left|k_{n}\right|+1\right)
$$

in $\pi_{\chi}$. For $l=\left(l_{1}, \ldots, l_{n}\right)$, let $F_{\chi}^{l}$ denote the automorphic form on $\mathrm{GL}_{2}\left(\mathbb{A}_{F}\right)$ corresponding to $\delta_{|k|+1}^{l} f_{\chi}$.

THEOREM 8.2. If $\chi$ does not factor through the norm map $\mathbb{A}_{E}^{\times} \rightarrow \mathbb{A}_{F}^{\times}$, we have

$$
\theta_{\phi_{l}^{\prime}}(\chi \xi)=D_{l} \cdot F_{\chi}^{l}, \quad \text { for some } D_{l} \neq 0 \text {. }
$$

Proof. First recall that by Theorem 6.15(a), the theta lift $\theta_{\phi^{\prime}}(\chi \xi)$ is an automorphic form in the automorphic induction $\pi_{\chi}$ to $\mathrm{GL}_{2}\left(\mathbb{A}_{F}\right)$. If $f$ is a Hecke eigenform of weight $|k|+1+2 l$ in $\pi_{\chi}$, then it must satisfy that for all $r(\theta):=r\left(\theta_{1}\right) \cdots r\left(\theta_{n}\right)$ with $r\left(\theta_{j}\right) \in \mathrm{SO}(2)$ and $k_{0}=\left(\begin{array}{ll}a & b \\ c & d\end{array}\right) \in K_{0}:=\prod_{v \nmid \infty} K_{0, v}$ with $\operatorname{det}\left(k_{0}\right)=1$, we have

$$
f\left(g r(\theta) d(\mathfrak{d})^{-1} k_{0} d(\mathfrak{d})\right)=\prod_{j=1}^{n} e^{i\left(\left|k_{j}\right|+1+2 l_{j}\right) \theta_{j}}\left(\chi \epsilon_{E / F}\right)(a) f(g) \text { for all } g \in \mathrm{GL}_{2}\left(\mathbb{A}_{F}\right) \text {. }
$$


By Casselman's theorem [Ca73, Theorem 1], the dimension of automorphic forms satisfying (8.2) must have dimension 1. Therefore to see that $\theta_{\phi^{\prime}}(\chi \xi)$ is a (possibly zero!) multiple of $F_{\chi}^{l}$, we need only see that it satisfies (8.2).

We first recall the definition of the theta lift $\theta_{\phi^{\prime}}(\chi \xi)$ on $\mathrm{GL}_{2}\left(\mathbb{A}_{F}\right)$. If $g \in$ $\mathrm{GL}_{2}\left(\mathbb{A}_{F}\right)^{+}:=\left\{g \in \mathrm{GL}_{2}\left(\mathbb{A}_{F}\right): \operatorname{det}(g) \in \operatorname{Nm}\left(\mathbb{A}_{E}^{\times}\right)\right\}$, then for any $h \in \mathbb{A}_{E}^{\times}$such that $\operatorname{det}(g)=\mathrm{Nm}(h)$,

$$
\theta_{\phi^{\prime}}(\chi \xi)(g)=\int_{\left[E^{1}\right]} \Theta\left(\omega_{\psi}\left(h h_{1}, g\right) \phi^{\prime}\right) \cdot(\chi \xi)\left(h h_{1}\right) d h_{1} .
$$

We define $\theta_{\phi^{\prime}}(\chi \xi)$ on

$$
\mathrm{GL}_{2}(F) \mathrm{GL}_{2}\left(\mathbb{A}_{F}\right)^{+}=\left\{g \in \mathrm{GL}_{2}\left(\mathbb{A}_{F}\right): \operatorname{det}(g) \in F^{\times} \mathrm{Nm}\left(\mathbb{A}_{E}^{\times}\right)\right\}
$$

by

$$
\theta_{\phi^{\prime}}(\chi \xi)(\gamma g)=\theta_{\phi^{\prime}}(\chi \xi)(g), \quad \text { for } \gamma \in \mathrm{GL}_{2}(F), g \in \mathrm{GL}_{2}\left(\mathbb{A}_{F}\right)^{+} \text {. }
$$

Note that $\mathrm{GL}_{2}(F) \mathrm{GL}_{2}\left(\mathbb{A}_{F}\right)^{+}$is an index-2 subgroup of $\mathrm{GL}_{2}\left(\mathbb{A}_{F}\right)$. We define $\theta_{\phi^{\prime}}(\chi \xi)$ on $\mathrm{GL}_{2}\left(\mathbb{A}_{F}\right)$ by extending by 0 outside $\mathrm{GL}_{2}(F) \mathrm{GL}_{2}\left(\mathbb{A}_{F}\right)$. Define $K_{0}:=$ $\prod_{v} K_{0, v}$, where $K_{0, v} \subset \mathrm{GL}_{2}\left(\mathcal{O}_{F_{v}}\right)$ as defined in Section 7. Note that $K_{0} \subset$ $\mathrm{GL}_{2}(F) \mathrm{GL}_{2}\left(\mathbb{A}_{F}\right)^{+}$. By Lemmas 7.3, 7.7, and 7.10, for $r(\theta)=r\left(\theta_{1}\right) \cdots r\left(\theta_{n}\right)$ with $r\left(\theta_{j}\right) \in \mathrm{SO}(2)$ and $k_{0}=\left(\begin{array}{ll}a & b \\ c & d\end{array}\right) \in K_{0} \cap \mathrm{GL}_{2}\left(\mathbb{A}_{F}\right)^{+}$,

$$
\omega_{\psi}\left(h_{0}, r(\theta) d(\mathfrak{d})^{-1} k_{0} d(\mathfrak{d})\right) \phi_{l}^{\prime}=\prod_{j=1}^{n} e^{i\left(\left|k_{j}\right|+1+2 l_{j}\right) \theta_{j}}(\chi \xi)^{-1}\left(h h_{0}\right)\left(\chi \epsilon_{E / F}\right)(a) \phi_{l}^{\prime},
$$

where $h_{0} \in \mathbb{A}_{E}^{\times}$is such that $\operatorname{Nm}\left(h_{0}\right)=\operatorname{det}\left(k_{0}\right)$. This implies that for any $g \in$ $\mathrm{GL}_{2}\left(\mathbb{A}_{F}\right)^{+}$and any $h \in \mathbb{A}_{E}^{\times}$with $\operatorname{Nm}(h)=\operatorname{det}(g)$,

$$
\begin{aligned}
& \theta_{\phi_{l}^{\prime}}(\chi \xi)\left(g r(\theta) d(\mathfrak{d})^{-1} k_{0} d(\mathfrak{d})\right) \\
&=\int_{\left[E^{1}\right]} \Theta\left(\omega_{\psi}\left(h h_{1} h_{0}, g r(\theta) d(\mathfrak{d})^{-1} k_{0} d(\mathfrak{d})\right) \phi_{l}^{\prime}\right) \cdot(\chi \xi)\left(h h_{1} h_{0}\right) d h_{1} \\
&=\prod_{j=1}^{n} \int_{\left[E^{1}\right]} \Theta\left(\omega_{\psi}\left(h h_{1}, g\right) \phi_{l}^{\prime}\right) \cdot e^{i\left(\left|k_{j}\right|+1+2 l_{j}\right) \theta_{j}} \\
& \cdot(\chi \xi)^{-1}\left(h_{0}\right) \cdot\left(\chi \epsilon_{E / F}\right)(a) \cdot(\chi \xi)\left(h h_{1} h_{0}\right) d h_{1} \\
&= \prod_{j=1}^{n} e^{i\left(\left|k_{j}\right|+1+2 l_{j}\right) \theta_{j}}\left(\chi \epsilon_{E / F}\right)(a) \cdot \int_{\left[E^{1}\right]} \Theta\left(\omega_{\psi}\left(h h_{1}, g\right) \phi_{l}^{\prime}\right) \cdot(\chi \xi)\left(h h_{1}\right) d h_{1} \\
&= \prod_{j=1}^{n} e^{i\left(\left|k_{j}\right|+1+2 l_{j}\right) \theta_{j}}\left(\chi \epsilon_{E / F}\right)(a) \cdot \theta_{\phi_{l}^{\prime}}(\chi \xi)(g) .
\end{aligned}
$$


In the next result, we give an exact formula (up to $\mathbb{C}^{1}$ ) for the constant $D_{l}$ in the case that $F=\mathbb{Q}$. One can do this for the general case by comparing Theorem 8.1 to known formulas for the Petersson inner product of Hilbert modular forms, but the formula for $D_{l}$ will be more complicated. In the following, we use [S76, Equation (2.5)], [H81, Section 5] together with the factors at bad places as determined in [Co18, Section 4.2].

Let $g$ be a twist of $f_{\chi}$, which is twist-minimal by $\chi_{g}$. Let $N, N_{g}$, be the levels of $f_{\chi}, g$, and let $N_{\chi, g}$ be the conductor of $\chi_{g}$. For every prime $p$, let $p^{r_{g}}$ be the exact power of $p$ dividing $N_{g}$, and $p^{r_{\chi_{g}}}$ be the exact power of $p$ dividing $N_{g, \chi}$. We denote by $L\left(s\right.$, ad, $\left.f_{\chi}\right)$ the adjoint $L$-function of $f_{\chi}$. Define

$$
\ell_{p}:= \begin{cases}1 & \text { if } p \nmid N, \\ (1+1 / p) L_{p}(\operatorname{ad}, f, 1) & \text { if } p \nmid N_{g} \text { and } p \mid N, \\ (1+1 / p) & \text { if } p \| N_{g} \text { and } p \| N, \\ (1+1 / p)\left(1-1 / p^{2}\right)^{-1} & \text { if } p \| N_{g} \text { and } p^{2} \mid N, \\ (1+1 / p) & \text { if } r_{g}=r_{\chi_{g}} \geqslant 1 \text { and } p^{r_{g}} \| N, \\ (1+1 / p)(1-1 / p)^{-1} & \text { if } r_{g}=r_{\chi_{g}} \geqslant 1 \text { and } p^{r_{g}+1} \mid N, \\ 1 & \text { if } r_{g} \geqslant 2 \text { and } r>r_{\chi_{g}} .\end{cases}
$$

Note that, comparing to [Co18, Section 4.2], the last case comes from the fact that $\pi_{\chi} \cong \pi_{\chi} \otimes \operatorname{det}\left(\varepsilon_{E / F}\right)$.

THEOREM 8.3. Assume $F=\mathbb{Q}$ and let $K_{0}$ be any maximal compact subgroup of $\mathrm{GL}_{2}\left(\mathbb{A}_{\mathbb{Q}, \text { fin }}\right)$ containing $K:=\prod_{v}, K_{0, v}\left(c_{v}\left(\pi_{\chi}\right)\right)$ (Sections 2.2 and 7$)$. Then

$$
\begin{aligned}
\left|D_{l}\right|^{2}= & \left|\frac{(2 \pi i)^{l}(2 i)^{l}}{(|k|+1)_{l}}\right|^{2} \cdot \rho_{E}^{-2} \cdot \prod_{v} C_{v} \cdot \zeta(2) \cdot(2 \pi)^{-1} \cdot 2 \\
& \cdot \frac{\left[K_{0}: K\right]}{\frac{\pi}{3} \cdot\left[\mathrm{PSL}_{2}(\mathbb{Z}): \Gamma_{1}(N)\right]} \cdot \frac{(4 \pi)^{|k|+1}}{|k| !} \cdot \prod_{p} \ell_{p} .
\end{aligned}
$$

In particular, $\left|D_{l}\right| \sim \pi^{l}$ and, up to an element of $\mathbb{C}^{1}, \theta_{\phi_{0}^{\prime}}(\chi \xi)$ is an algebraic holomorphic Hecke eigenform of weight $k+1$ and level $c(\chi)$.

Proof. By Lemma 7.4, we have

$$
\theta_{\phi_{l}^{\prime}}(\chi \xi)=\frac{(2 \pi i)^{l}(2 i)^{l}}{(|k|+1)_{l}} \cdot \delta_{|k|+1}^{l}\left(\theta_{\phi_{0}^{\prime}}(\chi \xi)\right) .
$$

It therefore suffices to calculate $\left|D_{0}\right|^{2}=\left\langle\theta_{\phi_{0}^{\prime}}(\chi \xi), \theta_{\phi_{0}^{\prime}}(\chi \xi)\right\rangle /\left\langle F_{\chi}, F_{\chi}\right\rangle$. 
Following [IP18+, Lemmas 6.1, 6.3], we have

$$
\left\langle F_{\chi}, F_{\chi}\right\rangle=(2 \pi) \cdot \zeta(2)^{-1} \cdot 2^{-1} \cdot\left[K_{0}: K\right]^{-1} \cdot \frac{\pi}{3}\left[\operatorname{PSL}_{2}(\mathbb{Z}): \Gamma_{1}(N)\right] \cdot\left\langle f_{\chi}, f_{\chi}\right\rangle,
$$

where $\left\langle f_{\chi}, f_{\chi}\right\rangle$ is normalized as in [S76, Equation (2.1)]: for any cusp form $f$ of weight $\kappa$ and level $\Gamma$, set

$$
\langle f, f\rangle:=\frac{1}{\operatorname{vol}(\mathfrak{h} / \Gamma)} \int_{\mathfrak{h} / \Gamma} f(x+i y) \overline{f(x+i y)} y^{k} \frac{d x d y}{y^{2}} .
$$

By [S76, Equation (2.5)], [H81, Section 5], and [Co18, Section 4.2],

$$
\left\langle f_{\chi}, f_{\chi}\right\rangle=\zeta(2)^{-1} \cdot \frac{|k| !}{(4 \pi)^{|k|+1}} \cdot \prod_{p \mid N} \ell_{p}^{-1} \cdot L\left(1, \mathrm{ad}, f_{\chi}\right) .
$$

We have $L\left(1\right.$, ad, $\left.f_{\chi}\right)=L(1, \tilde{\chi}) \cdot L\left(1, \varepsilon_{E / F}\right)=L(1, \tilde{\chi}) \cdot \rho_{E}$. Therefore, by Theorem 8.1,

$$
\left|D_{0}\right|^{2}=\rho_{E}^{-2} \cdot \prod_{v} C_{v} \cdot \zeta(2) \cdot(2 \pi)^{-1} \cdot 2 \cdot \frac{\left[K_{0}: K\right]}{\frac{\pi}{3} \cdot\left[\mathrm{PSL}_{2}(\mathbb{Z}): \Gamma_{1}(N)\right]} \cdot \frac{(4 \pi)^{|k|+1}}{|k| !} \cdot \prod_{p} \ell_{p} .
$$

Since $E$ is CM by construction so that $\rho_{E} \sim \pi$ and since $C_{\infty} \sim \pi^{2} / \pi^{|k|+1}$, we see

$$
\left|D_{0}\right|^{2} \sim \pi^{-2} \cdot \pi^{2} \cdot \pi^{2} \cdot \pi^{-1} \cdot \pi^{-1} \in \overline{\mathbb{Q}}
$$

\section{An example: the canonical Hecke character for $\mathbb{Q}(\sqrt{-7})$}

Let $F=\mathbb{Q}$ and let $E=\mathbb{Q}(\sqrt{-7})$. Then $E$ has class number 1 and there is a unique canonical character $\chi_{\text {can }}^{\prime}$ in the sense of Rohrlich [Ro80] (see also [Y95, page 52]). Explicitly, $\chi_{\text {can }}^{\prime}$ can be described as follows. First consider the character

$$
\epsilon: \mathcal{O}_{E} /(\sqrt{-7}) \cong \mathbb{Z} / 7 \mathbb{Z} \stackrel{(\dot{\overline{7}})}{\longrightarrow}\{ \pm 1\} .
$$

Then $\epsilon(-1)=-1$, and hence the map on principal ideals

$$
P(\sqrt{-7})=\left\{\alpha \mathcal{O}_{E}: \alpha \in E^{\times} \text {is relatively prime to } 7\right\} \rightarrow E^{\times}, \quad \alpha \mathcal{O}_{E} \mapsto \epsilon(\alpha) \alpha
$$

is a well-defined homomorphism. Since $E$ has class number 1 , then $P(\sqrt{-7})=$ $I(\sqrt{-7})$, and the above defines a Hecke character of $E^{\times}$. We define $\chi_{\text {can }}:=\chi_{\text {can }}^{\prime}$. $\|\cdot\|_{\mathbb{A}_{E}}^{1 / 2}$ to be the normalized unitary Hecke character of $E^{\times}$. It is easy to see that for $n>0$ :

(a) $\chi_{\text {can }}^{n}$ has $\infty$-type $(n, 0)+(-n / 2,-n / 2)=(n / 2,-n / 2)$.

(b) $\chi_{\text {can }}^{n}$ has conductor $\sqrt{-7} \mathcal{O}_{E}$ if $n$ is odd and conductor $\mathcal{O}_{E}$ if $n$ is even.

(c) $\left.\chi_{\mathrm{can}}\right|_{\mathbb{A}_{F}^{\times}}=\varepsilon_{E / F}$. 
9.1. Two quaternion algebras. We now compute the local epsilon factors $\epsilon_{v}\left(\mathrm{BC}\left(\pi_{\chi_{\text {can }}^{n}}\right) \otimes \chi_{\text {can }}^{m}\right)$. At $v=\infty$, this calculation depends on whether $n+1>m$ or $n+1 \leqslant m$. At the local places, this can be calculated using [T83, Section 1]. The interesting finite place is $v=7$.

(a) Momentarily, let $v$ be a real place of a number field $F$, take $f$ to be any automorphic form of $\mathrm{GL}_{2}$ of weight $k$ at $v$, and let $\Omega$ be a Hecke character of $E$ such that $\Omega_{v}(z)=z^{l_{1}} \bar{z}^{l_{2}}$. Then

$$
\epsilon_{v}(f, \Omega) \cdot \omega_{v}(-1)= \begin{cases}+1 & \text { if } k \leqslant l_{1}-l_{2}, \\ -1 & \text { if } k>l_{1}-l_{2}\end{cases}
$$

Since $\pi_{\chi_{\text {can }}^{n}}$ has weight $n+1$, this implies that

$$
\epsilon_{\infty}\left(\mathrm{BC}\left(\pi_{\chi_{\mathrm{can}}^{n}}\right) \otimes \chi_{\mathrm{can}}^{m}\right) \cdot \omega_{\infty}(-1)= \begin{cases}+1 & \text { if } n+1 \leqslant m, \\ -1 & \text { if } n+1>m .\end{cases}
$$

(b) Since $\chi_{\text {can, } v}$ factors through $\mathrm{Nm}$ for all $v \nmid 7$, the representation $\operatorname{Ind}_{\mathcal{W}_{E_{v}}}^{\mathcal{W}_{F_{v}}}\left(\chi_{\text {can }, v}\right)$ is decomposable. By [T83, Proposition 1.6], for any Hecke character $\Omega$, we have

$$
\epsilon_{v}\left(\mathrm{BC}\left(\pi_{\chi_{\mathrm{can}}}\right) \otimes \Omega\right) \cdot \omega_{v}(-1)=+1 \quad \text { for all } v \nmid 7 .
$$

(c) First, observe that $\operatorname{Res}_{\mathcal{W}_{E}} \operatorname{Ind}_{\mathcal{W}_{E}}^{\mathcal{W}_{F}}(\chi)=\chi \oplus \chi^{\tau}$ for any character $\chi$ of $\mathcal{W}_{E}$. Since base change on the $\mathrm{GL}_{2}$ side corresponds to restriction on the Galois side, we have

$$
\epsilon_{7}\left(\mathrm{BC}\left(\pi_{\chi \mathrm{can}}\right) \otimes \Omega\right)=\epsilon_{7}\left(\operatorname{Res}_{\mathcal{W}_{E}} \operatorname{Ind}_{\mathcal{W}_{E}}^{\mathcal{W}_{F}}(\chi) \otimes \Omega\right)=\epsilon_{7}\left(\chi_{\mathrm{can}} \Omega\right) \epsilon_{7}\left(\chi_{\mathrm{can}}^{\tau} \Omega\right),
$$

where the last equality holds because local $\epsilon$-factors change direct sums to products. By [Y95, Lemma 3.2], we have

$$
\epsilon_{7}\left(\chi_{\mathrm{can}} \Omega\right)=-\left(\frac{2}{7}\right) \sqrt{-1}=\epsilon_{7}\left(\chi_{\mathrm{can}}^{\tau} \Omega\right) .
$$

Since $\left.\chi_{\mathrm{can}}\right|_{F^{\times}}=\varepsilon_{E / F}$, the automorphic representation $\pi_{\mathrm{can}}$ has trivial central character, and hence the above calculation shows $\epsilon_{7}\left(\mathrm{BC}\left(\pi_{\chi_{\text {can }}}\right) \otimes\right.$ $\Omega) \omega_{7}(-1)=-1$. By the above argument,

$$
\epsilon_{7}\left(\mathrm{BC}\left(\pi_{\chi_{\text {can }}^{n}}\right) \otimes \chi_{\text {can }}^{m}\right) \cdot \omega_{7}(-1)= \begin{cases}+1 & \text { if } n \text { is even, } \\ -1 & \text { if } n \text { is odd } .\end{cases}
$$


We can now discuss the possibilities for the quaternion algebra determined by the pair of Hecke characters $\chi_{\text {can }}^{n}$ and $\chi_{\text {can }}^{m}$. First, observe that the central character condition $\chi_{\text {can }}^{n} \chi_{\text {can }}^{m} \varepsilon_{E / F}=1$ on $\mathbb{A}^{\times}$implies that $n$ and $m$ must have different parity. We now have two cases:

(i) If $n$ is odd, then $\epsilon_{v}\left(\mathrm{BC}\left(\pi_{\chi_{\text {can }}^{n}}\right) \otimes \chi_{\text {can }}^{m}\right)=-1$ if and only if $v=7$. This implies that if $L\left(\mathrm{BC}\left(\pi_{\chi_{\text {can }}^{n}}\right) \otimes \chi_{\text {can }}^{m}, \frac{1}{2}\right) \neq 0$, then necessarily $n+1>m$ so that $\epsilon_{\infty}\left(\mathrm{BC}\left(\pi_{\chi_{\text {can }}^{n}}\right) \otimes \chi_{\text {can }}^{m}\right)=-1$ and hence $\Sigma_{\pi_{\chi_{\text {can }}^{n}}^{n}, \chi_{\text {can }}^{m}}=\{7, \infty\}$.

(ii) If $n$ is even, then $\epsilon_{v}\left(\mathrm{BC}\left(\pi_{\chi_{\text {can }}^{n}}\right) \otimes \chi_{\text {can }}^{m}\right)=+1$ for all finite $v$. This implies that if $L\left(\mathrm{BC}\left(\pi_{\chi_{\text {can }}^{n}}\right) \otimes \chi_{\text {can }}^{m}, \frac{1}{2}\right) \neq 0$, then necessarily $n+1 \leqslant m$ so that $\epsilon_{\infty}\left(\mathrm{BC}\left(\pi_{\chi_{\text {can }}^{n}}\right) \otimes \chi_{\text {can }}^{m}\right)=+1$ and hence $\Sigma_{\pi_{\chi_{\text {can }}^{n}}, \chi_{\text {can }}^{m}}=\varnothing$.

Summarizing, take $n, m$ to have opposite parity. We have the following chart:

\begin{tabular}{c|c|c} 
& $n+1>m$ & $n+1 \leqslant m$ \\
& $\epsilon_{\infty}=-1$ & $\epsilon_{\infty}=+1$ \\
\hline$\epsilon=+1$ & $n$ odd, $m$ even & $n$ even, $m$ odd \\
& $\epsilon_{7}=-1$ & $\epsilon_{7}=+1$ \\
\hline$\epsilon=-1$ & (definite, ramified at $7, \infty)$ & (indefinite-in fact, split!) \\
\hline \multirow{2}{*}{$\epsilon_{7}=+1$} & $n$ odd, $m$ even \\
& $\epsilon_{7}=-1$
\end{tabular}

Waldspurger's formula is in the setting of $\epsilon=+1$, and our Main Theorem (Theorem 6.17) gives an identity between the two $\epsilon=+1$ boxes, taking $B=$ $M_{2}(F)$ and $B^{\prime}=B_{\{7, \infty\}}$. In Sections 7 and 8 , we constructed a family of Schwartz functions such that their theta lifts realize the newform and its images under iterates of the Shimura-Maass operator. We recall this construction next.

9.2. Torus periods of a weight- $(3+2 l) \mathbf{C M}$ form. Take the special case $n=2$ and let $m=3+2 l$, where $l \geqslant 0$. As in Section 7.1, we take $\phi_{l}^{\prime}:=\otimes_{v} \phi_{l, v}^{\prime}$, where

$$
\phi_{l, v}^{\prime}(z)= \begin{cases}{ }_{1} F_{1}(-l, 3,4 \pi z \bar{z}) z^{2} e^{-2 \pi z \bar{z}} & \text { if } v \mid \infty, \\ \mathbb{1}_{\mathcal{O}_{F_{v}}}\left(z_{1}\right) \cdot \mathbb{1}_{\mathcal{O}_{F_{v}}}\left(z_{2}\right) & \text { if } v \nmid \infty .\end{cases}
$$

If we set $\xi=\chi_{\text {can }}$,

$$
C_{v}= \begin{cases}\frac{(2 \pi)^{2}}{4^{3} \pi^{4}} \cdot \frac{l ! \cdot 4}{(l+2) !}=\frac{1}{2(l+2)(l+1) \pi^{2}} & \text { if } v \mid \infty \\ 1 & \text { if } v \neq 7 \\ \frac{1}{7}\left(1-49^{-1}\right)^{-1}\left(1-7^{-1}\right)=\frac{1}{8} & \text { if } v=7\end{cases}
$$


so that by Theorems 6.15(b) and 8.2, the theta lift $\theta_{\phi_{l}^{\prime}}\left(\chi_{\text {can }}^{2} \xi\right)$ is a Hecke eigenform on $\mathrm{GL}_{2}\left(\mathbb{A}_{\mathbb{Q}}\right)$ in $\pi_{\chi_{\text {can }}^{2}}$. Furthermore, again by Theorem 8.1,

$$
\left\langle\theta_{\phi_{l}^{\prime}}\left(\chi_{\mathrm{can}}^{2} \xi\right), \theta_{\phi_{l}^{\prime}}\left(\chi_{\mathrm{can}}^{2} \xi\right)\right\rangle=\left(\frac{2 \pi}{\sqrt{7} \cdot 2}\right)^{-1} \cdot \frac{1}{16 \cdot(l+2) \cdot(l+1) \cdot \pi^{2}} \cdot \frac{L\left(1, \tilde{\chi}_{\mathrm{can}}^{2}\right)}{\zeta(2)} .
$$

And as before, by Theorem 6.16,

$$
\int_{\left[E^{\times}\right]} \theta_{\phi_{l}^{\prime}}\left(\chi_{\mathrm{can}}^{2} \cdot \xi\right)(g) \cdot \chi_{\mathrm{can}}^{3+2 l}(g) d g=\int_{\left[E^{\times}\right]} \chi_{\mathrm{can}}^{2}(g) \cdot \overline{\theta_{\phi_{l}^{\prime}}^{\prime}\left(\overline{\chi_{\mathrm{can}}^{3+2 l} \cdot \xi^{\prime-1}}\right)}(g) d g,
$$

where by Theorem $6.15(\mathrm{~b})$ the theta lift $\overline{\theta_{\phi_{l}^{\prime}}^{\prime}\left(\overline{\chi_{\mathrm{can}}^{3+2 l} \cdot \xi^{\prime-1}}\right)}$ is an automorphic form in $\pi_{\chi_{\text {can }}^{3+2 l}}^{B^{\prime}}$.

9.3. Nonvanishing torus periods. Recall that by Theorem $8.2, \theta_{\phi_{l}^{\prime}}\left(\chi_{\mathrm{can}}^{2} \xi\right)$ is a nonzero Hecke eigenform of weight $3+2 l$ in $\pi_{\chi_{\text {can }}^{2}}$. For a basis $1, \mathbf{i}, \mathbf{j}$, ij of $M_{2}(\mathbb{Q})$ with $\mathbf{i}^{2}=u=-7, \mathbf{j}^{2}=-1 / 7$,

$$
E^{\times} \hookrightarrow \mathrm{GL}_{2}(\mathbb{Q}), \quad a+b \mathbf{i} \mapsto\left(\begin{array}{cc}
a & -2 b \\
-b u / 2 & a
\end{array}\right) .
$$

In particular, for any finite place $p$ of $\mathbb{Q}$, the induced embedding $E_{p}^{\times} \hookrightarrow \mathrm{GL}_{2}\left(\mathbb{Q}_{p}\right)$ makes

$$
K_{0, p}:= \begin{cases}\mathrm{GL}_{2}\left(\mathbb{Z}_{p}\right) & \text { if } p \neq 7, \\
\left\{\left(\begin{array}{ll}
a & b \\
c & d
\end{array}\right) \in \mathrm{GL}_{2}\left(\mathbb{Z}_{7}\right): c \in 7 \mathbb{Z}_{7}\right\} & \text { if } p=7\end{cases}
$$

an optimal compact open subgroup (in the sense of Gross [G88, Proposition 3.2]) with respect to $\chi_{\text {can }}^{3+2 l}$, which is unramified at $v \nmid 7$ and has conductor 1 at $v \mid 7$. By [GP91, Proposition 2.3], since $\chi_{\text {can }}^{2}$ is unramified at every place $p$ of $\mathbb{Q}$, a Hecke eigenform with respect to the above compact open subgroup of $\mathrm{GL}_{2}\left(\mathbb{A}_{\mathbb{Q}}\right)$ is locally (up to a scalar) the Gross-Prasad test vector. By Waldspurger,

$$
\int_{\left[E^{\times}\right]} \theta_{\phi_{l}^{\prime}}\left(\chi_{\text {can }}^{2} \xi\right)(g) \cdot \chi_{\text {can }}^{3+2 l}(g) d g \neq 0 \quad \Longleftrightarrow \quad L\left(\mathrm{BC}\left(\pi_{\chi_{\text {can }}^{2}}\right) \otimes \chi_{\text {can }}^{3+2 l}, \frac{1}{2}\right) \neq 0 .
$$

Combining this with Theorems 6.17, 8.2, and 8.3, we obtain the following corollary.

COROLlaRY 9.1. Let $B^{\prime}=B_{\{7, \infty\}}$ denote the definite quaternion algebra over $\mathbb{Q}$ ramified at exactly 7 and $\infty$. Define

$$
f_{\chi_{\text {can }}^{2}}^{(l)}:=\theta_{\phi_{l}^{\prime}}\left(\chi_{\mathrm{can}}^{2} \xi\right), \quad f_{\chi_{\mathrm{can}}^{3+2 l}}^{B^{\prime}}:=\overline{\theta_{\phi_{l}^{\prime}}^{\prime}\left(\overline{\chi_{\mathrm{can}}^{3+2 l} \xi^{\prime}}\right)} .
$$


Then, we have the following:

(a) $\pi^{-l} \cdot f_{\chi_{\mathrm{can}}^{2}}^{(l)}$ is an algebraic Hecke eigenform of weight $3+2 l$ in $\pi_{\chi_{\mathrm{can}}^{2}}$.

(b) $f_{\chi_{\mathrm{can}}^{3+2 l}}^{B^{\prime}}$ is an automorphic form in the Jacquet-Langlands transfer $\pi_{\chi_{\mathrm{can}}^{3+2 l}}^{B^{\prime}}$.

(c) There is an identity of torus periods

$$
\int_{\left[E^{\times}\right]} f_{\chi_{\text {can }}^{2}}^{(l)}(g) \cdot \chi_{\text {can }}^{3+2 l}(g) d g=\int_{\left[E^{\times}\right]} \chi_{\text {can }}^{2}(g) \cdot f_{\chi_{\text {can }}^{3+2 l}}^{B^{\prime}}(g) d g,
$$

nonzero if and only if the central value $L\left(\mathrm{BC}\left(\pi_{\chi_{\text {can }}^{2}}\right) \otimes \chi_{\mathrm{can}}^{3+2 l}, \frac{1}{2}\right)$ is nonvanishing.

Note in particular that if $L\left(\mathrm{BC}\left(\pi_{\chi_{\mathrm{can}}^{2}}\right) \otimes \chi_{\mathrm{can}}^{3+2 l}\right) \neq 0$, then the automorphic form $f_{\chi_{\operatorname{can}}^{3+2 l}}^{B^{\prime}}$ on the definite quaternion algebra $B^{\prime}$ is a test vector for the Waldspurger torus period for the cuspidal representation $\pi_{\chi_{\mathrm{can}}^{3+2 l}}^{B^{\prime}}$ paired with $\chi_{\mathrm{can}}^{2}$.

\section{Acknowledgements}

I would like to thank my advisor Kartik Prasanna for introducing me to this area of research and Atsushi Ichino for many helpful conversations. A further thank you goes to both Kartik and Atsushi for sharing their impeccably written preprints with me at an early stage. This work was partially supported by NSF grants DMS-0943832, DMS-1160720, and DMS-1802905.

Conflict of Interest: The author declares that there is no conflict of interest.

\section{References}

[BDP13] M. Bertolini, H. Darmon and K. Prasanna, 'Generalized Heegner cycles and p-adic Rankin $L$-series. With an appendix by Brian Conrad', Duke Math. J. 162(6) (2013), 1033-1148.

[Ca73] W. Casselman, 'On some results of Atkin and Lehner', Math. Ann. 201 (1973), 301-314.

[Ch18] C. Chan, 'Period identities of CM forms on quaternion algebras', PhD Thesis, University of Michigan, 2018.

[Co18] D. Collins, 'Numerical computation of Petersson inner products and $q$-expansions', Preprint, 2018, arXiv:1802.09740.

[GI14] W. T. Gan and A. Ichino, 'Formal degrees and local theta correspondence', Invent. Math. 195(3) (2014), 509-672.

[GQT] W. T. Gan, Y. Qiu and S. Takeda, 'The regularized Siegel-Weil formula (the second term identity) and the Rallis inner product formula', Invent. Math. 198(3) (2014), 739-831. 
[G88] B. Gross, 'Local orders, root numbers, and modular curves', Amer. J. Math. 110 (1988), 1153-1182.

[GP91] B. Gross and D. Prasad, 'Test vectors for linear forms', Math. Ann. 291(2) (1991), 343-355.

[HKS96] M. Harris, S. Kudla and W. Sweet, 'Theta dichotomy for unitary groups', J. Amer. Math. Soc. 9(4) (1996), 941-1004.

[H81] H. Hida, 'Congruence of cusp forms and special values of their zeta functions', Invent. Math. 63(2) (1981), 225-261.

[IP18+] A. Ichino and K. Prasanna, 'Periods of quaternionic Shimura varieties', Mem. Amer. Math. Soc, to appear.

[IP18++] A. Ichino and K. Prasanna, 'Periods of quaternionic Shimura varieties, II', Preprint, 2020.

[JL] H. Jacquet and R. Langlands, Automorphic Forms for $\mathrm{GL}_{2}$, Lecture Notes in Mathematics, 114 (Springer, Berlin, New York, 1970), vii + 548.

[K94] S. Kudla, 'Splitting metaplectic covers of dual reductive pairs', Israel J. Math. 87(1-3) (1994), 361-401.

[KS97] S. Kudla and W. Sweet, 'Degenerate principal series representations for $U(n, n)$, Israel J. Math. 98 (1997), 253-306.

[LR05] E. M. Lapid and S. Rallis, 'On the local factors of representations of classical groups', in Automorphic Representations, L-Functions and Applications: Progress and Prospects, Ohio State University Mathematics Research Institute, 11 (de Gruyter, Berlin, 2005), 309-359.

[MVW] C. Moeglin, M.-F. Vignéras and J.-L. Waldspurger, Correspondances de Howe sur Uncorps p-Adique, Lecture Notes in Mathematics, 1291 (Springer, Berlin, 1987), viii +163 .

[PSR87] I. Piatetski-Shapiro and S. Rallis, ' $L$-functions for the classical groups', in Modular Forms (Durham, 1983), Ellis Horwood Ser. Mat. Appl: Statist. Oper. Res. (Horwood, Chichester, 1984), 251-261.

[P06] A. Popa, 'Central values of Rankin $L$-series over real quadratic fields', Compos. Math. 142 (2006), 811-866.

[P93] D. Prasad, 'Weil representation, Howe duality, and the theta correspondence', in Theta Functions: from the Classical to the Modern, CRM Proc. Lecture Notes (American Mathematical Society, Providence, RI, 1993), 105-127.

[R84] S. Rallis, 'On the Howe duality conjecture', Compos. Math. 51 (1984), 333-399.

[R93] R. Ranga Rao, 'On some explicit formulas in the theory of Weil representations', Pacific J. Math. 157(2) (1993), 335-371.

[Ro80] D. Rohrlich, 'On the $L$-function of canonical Hecke characters of imaginary quadratic fields', Duke Math. J. 47(3) (1980), 547-557.

[S93] H. Saito, 'On Tunnell's formula for characters of GL(2)', Compos. Math. 85(1) (1993), 99-108.

[S76] G. Shimura, 'The special values of the zeta functions associated with cusp forms', Comm. Pure Appl. Math. 29(6) (1976), 783-804.

[SZ15] B. Sun and C.-B. Zhu, 'Conservation relations for local theta correspondence', J. Amer. Math. Soc. 28(4) (2015), 939-983.

[T83] J. Tunnell, 'Local $\epsilon$-factors and characters of GL(2)', Amer. J. Math. 105(6) (1983), 1277-1307.

[VK91] N. J. Vilenkin and V. U. Klimyk, Representations of Lie Groups and Special Functions, Vol. 1, Mathematics and its Applications (Soviet Ser.), 72 (Kluwer Academic, Dordrecht, 
1991).

[W85a] J.-L. Waldspurger, 'Sur les valeurs de certaines fonctions $L$ automorphes en leur centre de symétrie', Compos. Math. 54(2) (1985), 173-242.

[X07] H. Xue, 'Central values of $L$-functions over CM fields', J. Number Theory 122 (2007), 342-378.

[Y95] T. Yang, 'Theta liftings and the $L$-function of elliptic curves', PhD Thesis, University of Maryland, College Park, 1995, 126 pages, ProQuest LLC. 